\title{
Synthesis and Electrochemical and Spectroscopic Characterization of 4,7-diamino-1,10-phenanthrolines and Their Precursors
}

\author{
Jacek E. Nycz ${ }^{1, *(1)}$, Jakub Wantulok ${ }^{1}$, Romana Sokolova ${ }^{2}{ }^{1}$, Lukasz $^{\text {Pajchel }}{ }^{3}{ }^{\mathbb{C}}$, \\ Marek Stankevič ${ }^{4}$, Marcin Szala ${ }^{5}$, Jan Grzegorz Malecki ${ }^{1}$ (D) and Daniel Swoboda ${ }^{1}$ \\ 1 Institute of Chemistry, University of Silesia in Katowice, ul. Szkolna 9; PL-40007 Katowice, Poland; \\ jakub.wantulok1@gmail.com (J.W.); jan.malecki@us.edu.pl (J.G.M.); daniel.swoboda96@gmail.com (D.S.) \\ 2 J. Heyrovský Institute of Physical Chemistry of the Czech Academy of Sciences, Dolejškova 3, 18223 Prague, \\ Czech Republic; romana.sokolova@jh-inst.cas.cz \\ 3 Department of Analytical Chemistry and Biomaterials, Faculty of Pharmacy, Medical University of Warsaw, \\ Banacha 1, 02-097 Warsaw, Poland; lpajchel@wum.edu.pl \\ 4 Department of Organic Chemistry, Marie Curie-Sklodowska University, 33 Gliniana St, PL-20614 Lublin, \\ Poland; marek.stankevic@poczta.umcs.lublin.pl \\ 5 Institute of Polymer and Dye Technology, Lodz University of Technology, Stefanowskiego 12/16, 90-924 \\ Lodz, Poland; marcin.szala@p.lodz.pl \\ * Correspondence: jacek.nycz@us.edu.pl; Tel.: +48-32-359-1446
}

Academic Editor: Fawaz Aldabbagh

Received: 23 October 2019; Accepted: 8 November 2019; Published: 13 November 2019

\begin{abstract}
New approaches to the synthesis of 4,7-dichloro-1,10-phenanthrolines and their corresponding $9 \mathrm{H}$-carbazol-9-yl-, $10 \mathrm{H}$-phenothiazin-10-yl- and pyrrolidin-1-yl derivatives were developed. Their properties have been characterized by a combination of several techniques: MS, HRMS, GC-MS, electronic absorption spectroscopy and multinuclear NMR in both solution and solid state including ${ }^{15} \mathrm{~N} \mathrm{CP} / \mathrm{MAS}$ NMR. The structures of 5-fluoro-2,9-dimethyl-4,7-di(pyrrolidin-1-yl)-1, 10-phenanthroline (5d), 4,7-di(9H-carbazol-9-yl)-9-oxo-9,10-dihydro-1,10-phenanthroline-5-carboni trile (6a) and 4,7-di(10H-phenothiazin-10-yl)-1,10-phenanthroline-5-carbonitrile (6b) were determined by single-crystal X-ray diffraction measurements. The nucleophilic substitutions of hydrogen followed by oxidation produced compounds $\mathbf{6 a}$ and $\mathbf{6 b}$. The electrochemical properties of selected 1,10-phenanthrolines were investigated using cyclic voltammetry and compared with commercially available reference 1,10-phenanthrolin-5-amine (51). The spatial distribution of frontier molecular orbitals of the selected compounds has been calculated by density functional theory (DFT). It was shown that potentials of reduction and oxidation were in consistence with the level of HOMO and LUMO energies.
\end{abstract}

Keywords: phenanthroline; amination; cyclic voltammetry; ${ }^{15} \mathrm{~N}-\mathrm{NMR}$; cyclization; heterocyclic; $\mathrm{DFt}$; oxidative nucleophilic substitutions of hydrogen

\section{Introduction}

1,10-Phenanthrolines are well known bidentate nitrogen ligands used in coordination chemistry for the analysis of transition metal ions and homogenous catalysis [1-4]. Their coordination abilities are the result of the contribution of lone pairs of electrons on both nitrogen atoms in the characteristic shape of heterotricyclic systems. They have been found applications in supramolecular chemistry, luminescent sensors, and photosensitizers for solar cells [5-15]. 1,10-Phenanthrolines have also received much attention as potential targets for anticancer drug development [16-21]. Mainly there are two methods for the synthesis of compounds containing 1,10-phenanthroline 
core. The first one is the classical Skraup and Friedlander transformations. The second method is used in the synthesis of 4,7-dichloro-1,10-phenanthroline derivatives through the condensation of Meldrum's acid, orthoesters, and ortho-phenylenediamine derivatives, followed by sequential thermal cyclization, decarboxylation and treatment with refluxing phosphoryl chloride [22,23]. The synthesis of 4,7-dichloro-1,10-phenanthrolines offered a possibility of further transformation through replacing halogen atoms in C4 and C7 positions. In the literature there are few methods describing their modification into amino derivatives [24,25]. In this paper, we report new approaches to the synthesis of the aforementioned compounds with in-depth spectroscopic and electrochemical characterization. In order to improve the understanding of the chemical behavior of novel 4,7-dichloro-1,10-phenanthrolines and their amino derivatives, a ${ }^{15} \mathrm{~N} \mathrm{CP} / \mathrm{MAS} \mathrm{NMR}$ technique was employed to differentiate and characterize the nitrogen atoms presented in their structures, and to show the influence of substituents on their chemical shifts. Additionally, computation and X-ray studies have been carried out of on the novel 4,7-disubstituted-1,10-phenanthrolines. The investigation of the electrochemical properties of these newly designed compounds may provide a promising tool to demonstrate their stability towards oxidative and reductive conditions for applications, such as advanced synthesis and catalysis.

\section{Results}

\subsection{Synthesis of 4,7-dichloro-1,10-phenanthrolines}

In the current study, the functionalization of selected 1,10-phenanthroline derivatives at $\mathbf{R}, \mathbf{R}^{\mathbf{1}}$ and $\mathbf{R}^{2}$ positions was the focus, which have not been fully exploited and may serve as interesting building blocks. The classical Skraup-Doebner-Miller reaction offers the simplest and fastest transformation. To prepare 1,10-phenanthrolines we first attempted to adopt a one-step Skraup-Doebner-Miller cyclocondensation of ortho-phenylenediamines 1 with unsaturated carbonyl compounds. However, like in previous work by other authors, this procedure failed, possibly due to an intermolecular condensation [26]. Instead, the synthesis of 4,7-dichloro-1,10-phenanthroline derivatives 4 has been carried out using a three-step condensation of 2,2-dimethyl-1,3-dioxane-4,6-dione (Meldrum's acid), orthoesters, and ortho-phenylenediamines 1 (Scheme 1) [22,23]. Compounds 4 were prepared with high yield, even on the scale of several grams. The first yield-determining step depends on the double nucleophilic addition of ortho-phenylenediamines (1) to the vinyl group of an intermediate obtained from the condensation of Meldrum's acid and an orthoester (Scheme 2).

Anilines are poor $N$-nucleophiles. Their reactivity strongly depends on the solvents used, and substituents in their structures. In a series of experiments it was noticed that a higher isolation yield (up to $94 \%$ ) was obtained for molecule $\mathbf{2} \mathbf{d}$ which possesses a methyl group as $\mathbf{R}^{\mathbf{1}}$ substituent and hydrogen atoms as $\mathbf{R}$ (Scheme 2). Replacing the methyl group by a halogen atom $(\mathrm{F}, \mathrm{Cl}, \mathrm{Br})$ or an electron-withdrawing group like $\mathrm{CN}$ or $\mathrm{COOH}$ in aniline decreases the isolated yield. Additionally, it is observed that the yield of products was dependent on the orthoester used. The use of trimethyl orthoformate produced the highest yields of compounds $\mathbf{2 a}, \mathbf{2 b}, \mathbf{2 c}, \mathbf{2 d}$ and $\mathbf{2 g}$, followed by a lower yield using triethyl orthoacetate, and the lowest was triethyl orthoformate of molecules $\mathbf{2} \mathbf{f}, \mathbf{2} \mathbf{h}, \mathbf{2} \mathbf{i}, \mathbf{2} \mathbf{j}$ and 21. This reactivity could be explained by increased steric interactions between Meldrum's acid and the orthoesters, which hampers the transformation (Scheme 2). In the syntheses of heterocycles 2, appropriate esters were produced depending on the ortho esters used as reactants. The hydrogen atom in carboxyl group was replaced by methyl or ethyl fragment giving the compounds $\mathbf{2 m}$ and $\mathbf{2 n}$, respectively. It is of importance that the synthesis of products 2 was accompanied by side reactions which led to benzo[d]imidazole type molecules, for example during the synthesis of compound $\mathbf{2 f}$ $1 H$-benzo[d]imidazole-6-carboxylic acid was isolated. During the next step, diketene was generated in situ from molecule 2 . This process relies on a thermally induced decarboxylation and acetone elimination. Highly reactive ketene underwent a cyclocondensation with the aryl substituent leading to compound 3 (Scheme 2). It is noticed that the rate of this reaction was not very sensitive to the presence of substituents at the C5 position $\left(\mathbf{R}^{\mathbf{1}}\right)$. This method is suitable for a variety of useful 
functional groups, including ester, $\mathrm{CN}$, alkyl substituents and halogen atoms, with the exception however of $\mathrm{COOH}$, which reacted with electrophilic ketene through disturbing cyclocondensation to give very complicated reaction mixture. Carbonyl groups at $C 4$ and $C 7$ positions in heterocycle 3 can be replaced by chlorine atoms to prepare 4,7-dichloro-1,10-phenanthrolines 4 . In a series of experiments, molecule 3 was treated with phosphoryl chloride giving quantitatively products 4 (Scheme 1) [22,23]. 4,7-Dichloro-1,10-phenanthrolines were obtained in high yields (ca. 68-93\%) with the exception of compounds $4 \mathbf{e}(48 \%), 41(32 \%)$ and $4 \mathrm{~m}(38 \%)$. The lower yields of these molecules could be explained by the hydrolysis of their $\mathbf{R}^{\mathbf{1}}$ substituents, i.e., the $\mathrm{CN}$, COOMe and COOEt groups and formation of carboxylic acid groups [27]. To avoid the hydrolysis of $\mathrm{CN}$ and COOAlk groups, and a reverse reaction of the 4,7-dichloro-1,10-phenanthrolines 4 to form the 1,10-dihydro-1,10-phenanthroline-4,7-diones 3 , the excess of phosphoryl chloride should be evaporated under reduced pressure. This strategy should facilitate hydrolysis of the excess of phosphoryl chloride. It is noticed that the use of basic solutions during the hydrolysis procedure causes the competitive reversion of product 4 to molecule 3. Hydroxide ion can substitute for chloride atom at the $\mathrm{C} 4 / \mathrm{C} 7$ positions. Surprisingly during the isolation of compound $4 \mathbf{e}$ an unexpected product $\mathbf{4 f}$ was obtained, which is explained as a hydrolysis of the heterocycle 4e. It is worth noting that during this chemical transformation 4,7-dichloro-1,10-phenanthroline-5-carboxylic acid was not isolated or identified (Scheme 3).

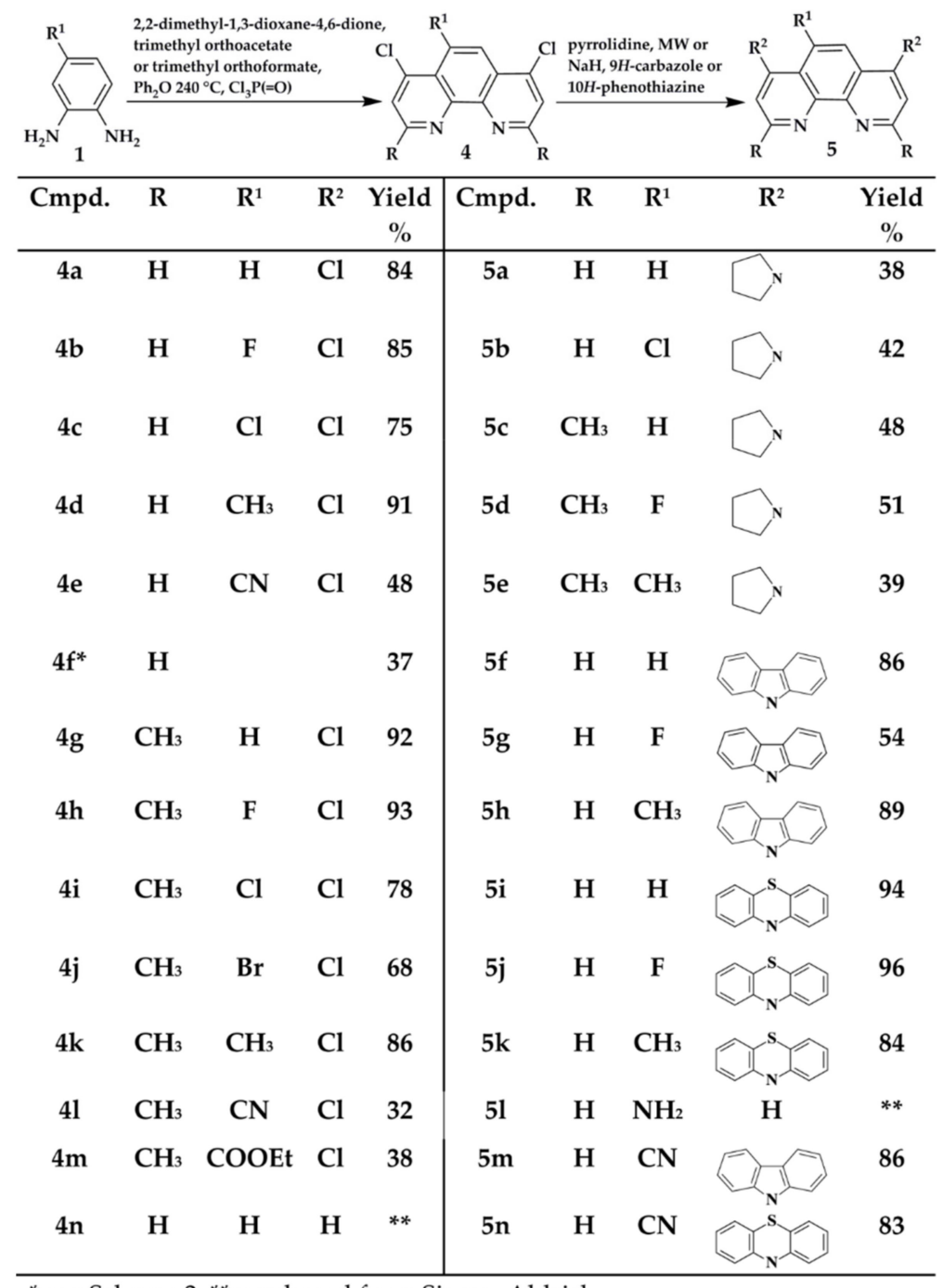

* see Scheme 3, ${ }^{* *}$ purchased from Sigma-Aldrich

Scheme 1. Synthesis route of compounds 4 and 5. 


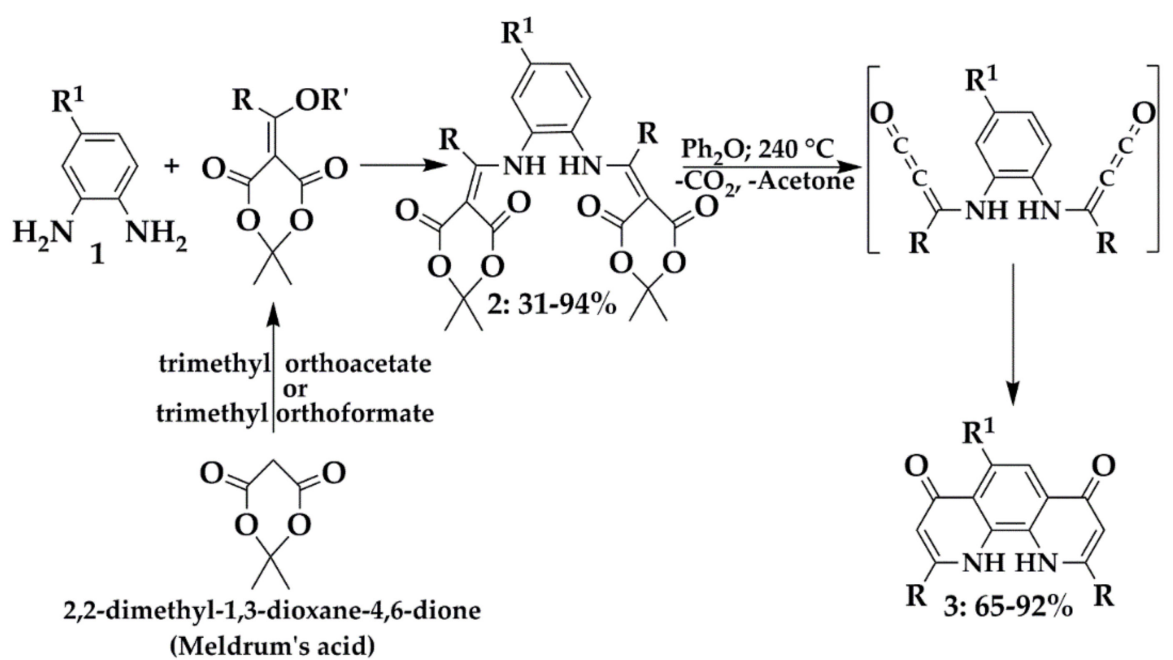

Scheme 2. Synthesis route of the compounds $\mathbf{3} . \mathbf{R}=\mathrm{Me}, \mathrm{Et} . \mathbf{R}^{\mathbf{1}}=\mathrm{H}, \mathrm{F}, \mathrm{Cl}, \mathrm{Br}, \mathrm{Me}, \mathrm{CN}$.

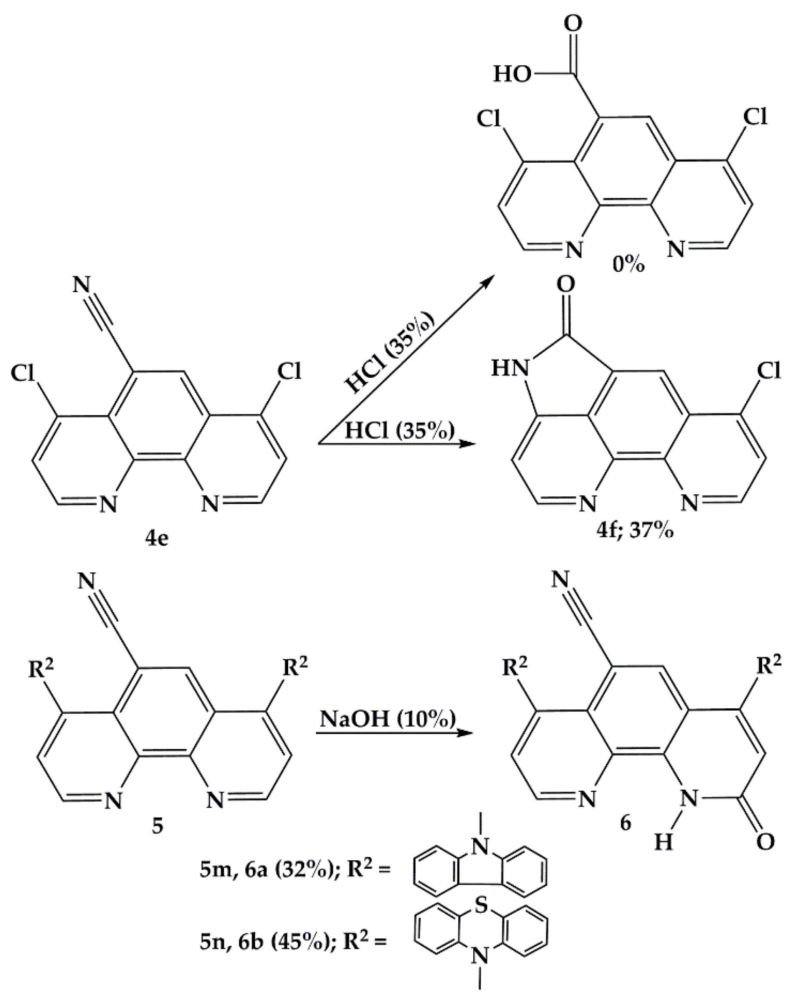

Scheme 3. Complexity of the hydrolysis reactions of the $\mathrm{CN}$ group.

\subsection{Synthesis of 4,7-diamino-1,10-phenanthrolines}

The introduction of substituents $\mathbf{R}^{\mathbf{2}}$, such as a chlorine atom in the 1,10-phenanthroline skeleton, opened opportunities for the further functionalization. Five 4,7-dipyrrolidinyl-1,10-phenanthrolines 5 with four novel structures were synthesized from heterocycles 4 by microwave-assisted nucleophilic aromatic substitution with a 10-fold excess of pyrrolidine. The nitrogen $\mathbf{R}^{2}$ substituents should increase the electron density on nitrogen atoms in 1,10-phenanthrolines (Scheme 1) [24]. Reactions were carried out in a sealed vial in a microwave reactor at $130^{\circ} \mathrm{C}$ (Scheme 1). MW irradiation improves the reactivity of the substitutions, shortening the reaction time to give the substitution products in better yields. In the absence of MW irradiation, the substitution reaction failed to initiate. The yields of products depend on the substituent $\mathbf{R}^{\mathbf{1}}$, which may influence the outcome of the reaction through steric and/or electronic effect. Unexpectedly $\mathbf{R}^{\mathbf{1}}$ fluorine or chlorine atoms failed to undergo substitution. This 
phenomenon can be explained by a steric hindrance attributed to the already introduced pyrrolidinyl rings and too high an electron density on the carbon atom at C5 position. Substituents $\mathbf{R}^{2}$ in the pyridine rings in 1,10-phenanthrolines 4 tended to undergo more $S_{N}$ Ar type substitutions due to the presence of nitrogen atoms which are able to accommodate a negative charge in the intermediate state.

Four 4,7-di(9H-carbazol-9-yl)-1,10-phenanthrolines (5f, 5g, $5 \mathbf{h}$ and $5 \mathbf{m})$ and four 4,7-di(10H-phen othiazin-10-yl)-1,10-phenanthroline derivatives (5i, 5j, 5k and $5 \mathbf{n})$ with seven novel structures were synthesized from molecules 4 by nucleophilic aromatic substitution with $9 H$-carbazole and 10H-phenothiazine, respectively (Scheme 1). Reagents were stirred for sixteen hours under reflux. The chemistry was based on inexpensive, commercially available reagents and easily synthesized heterocycles $\mathbf{4}$ which possess methyl, $\mathrm{CN}$, fluorine or hydrogen atom as $\mathbf{R}^{\mathbf{1}}$. The yields of $9 \mathrm{H}$-carbazole derivatives $\mathbf{5}$ were lower than for $10 \mathrm{H}$-phenothiazine derivatives. In all cases the purification of $9 \mathrm{H}$-carbazole derivatives 5 required chromatographic methods to receive pure products. However, the purification of $10 \mathrm{H}$-phenothiazine could be carried out by crystallization from the mixture of $\mathrm{CH}_{2} \mathrm{Cl}_{2}$ (or THF) and hexane to afford products even on a multigram scale. The highest yield and less complex reaction mixture of $10 \mathrm{H}$-phenothiazine derivatives can be attributed to the better nucleophilic properties of $10 \mathrm{H}$-phenothiazine anion than $9 \mathrm{H}$-carbazole. Wu et al. obtained the X-ray structure of 4,7-di(10H-phenoxazin-10-yl)-1,10-phenanthroline [28], which was cocrystallized with THF as a guest molecule located in the crystalline frameworks. The THF molecule is surrounded by two $10 \mathrm{H}$-phenoxazine rings and 1,10-phenanthroline core. ${ }^{1} \mathrm{H}-\mathrm{NMR}$ and ${ }^{13} \mathrm{C}-\mathrm{NMR}$ studies showed that the presence of signals from THF for $10 H$-phenothiazine derivatives $5 \mathbf{i}, \mathbf{5} \mathbf{j}$ and $\mathbf{5 n}$ (Supporting Information). It is worth noting that in the case of molecule $5 \mathbf{k}$, with a methyl group as $\mathbf{R}^{\mathbf{1}}$, no residual THF signals have been detected. $9 \mathrm{H}$-Carbazole derivatives have shown similar supramolecular inclusion processes to compounds $\mathbf{5 i}, \mathbf{5} \mathbf{j}$ and $\mathbf{5 n}$, in which the solvent is located in a cage made by 1,10-phenanthroline derivatives, however, with smaller intensity.

Yields of selected 1,10-phenanthrolines 4 and 5 are summarized in Scheme 1. 4,7-Di(10H-pheno thiazin-10-yl)-1,10-phenanthroline derivatives were obtained with yields in the range $84-96 \%$. 4,7-Disubstituted-1,10-phenanthrolines with a carboxylic acid group as $\mathbf{R}^{\mathbf{1}}$ substituent should be readily soluble in waters. These bifunctional compounds should mimic amino acids or nicotinic acid. Therefore, in order to synthesize appropriate carboxylic acids we carried out the hydrolysis of the $\mathrm{CN}$ group in molecule 4e, using an excess of acid (ca. 50.0 equiv. $\mathrm{HCl}$ ) or excess of base (ca. 50.0 equiv. $\mathrm{NaOH}$ ) in the case of 4,7-di(9H-carbazol-9-yl)-1,10-phenanthroline-5-carbonitrile (5m) and 4,7-di(10H-phenothiazin-10-yl)-1,10-phenanthroline-5-carbonitrile (5n) under the applied conditions. It is shown in Scheme 3 that in both cases the reactions does not lead to the formation of the expected 4,7-dichloro-1,10-phenanthroline-5-carboxylic acid or related molecules as was observed for the synthesis of 1,10-phenanthroline-2-carboxylic acid [27]. We isolated unexpected product $\mathbf{4 f}$. The hydrolysis of heterocycles $\mathbf{5 m}$ and $\mathbf{5 n}$ lead to other surprising compounds, namely 4,7-di(9H-carbazol-9-yl)-9-oxo-9,10-dihydro-1,10-phenanthroline-5-carbonitrile (6a) and 4,7-di(10H-pheno- thiazin-10-yl)-1,10-phenanthroline-5-carbonitrile (6b). Both molecules were possibly obtained through a reaction of oxidative nucleophilic substitutions of hydrogen (ONSH) of the type $\mathrm{S}_{\mathrm{N}} \mathrm{Ar}^{\mathrm{H}}$, with hydroxide ion as a nucleophile and in the presence of air as oxidized reagent $[29,30]$. In both cases nucleophile attacks were selective only at $\mathrm{C} 2$ rather than at the $\mathrm{C} 9$ position. The natural atomic charges show that both heterocycles possess greater electron density on the C2 ( 0.040 for compound $5 \mathrm{~m}$, and 0.038 for compound $5 \mathrm{n})$ than on the C9 position $(0.046$ for compound $5 \mathrm{~m}$ and 0.044 for compound $\mathbf{5 n}$ ) (Figures S1 and S2; Supporting Information). Both compounds $\mathbf{6} \mathbf{a}$ and $\mathbf{6 b}$ were isolated in a crystalline form, and their structures were characterized by X-ray crystallography. The prepared 4,7-dichloro-1,10-phenanthrolines 4 tend to undergo a fragmentation with the initial loss of chloride, followed by the loss of a second chloride and further decomposition, with the exception of molecule $\mathbf{4} \mathbf{j}$, which first eliminates bromide. 


\subsection{X-Ray Studies}

Crystals of compounds $\mathbf{5 d}, \mathbf{6 a}$ and $\mathbf{6 b}$ were mounted in turn on a Gemini A Ultra Oxford Diffraction automatic diffractometer equipped with a CCD detector used for data collection. X-ray intensity data were collected with graphite monochromated $\mathrm{MoK}_{\alpha}$ radiation at room temperature, with $\omega$ scan mode. Details concerning crystal data and refinement are gathered in Table 1 in ESI. Lorentz, polarization and empirical absorption correction using spherical harmonics implemented in SCALE3 ABSPACK scaling algorithm were applied [31]. The structures were solved by a direct method and subsequently completed by a difference Fourier recycling. All the non-hydrogen atoms were refined anisotropically using full-matrix, least-squares techniques. The Olex2 [32] and SHELXS, SHELXL [33] programs were used for all the calculations. Atomic scattering factors were incorporated in the computer programs.

Table 1. Chemical structures and HOMO and LUMO distribution of selected compounds.

If

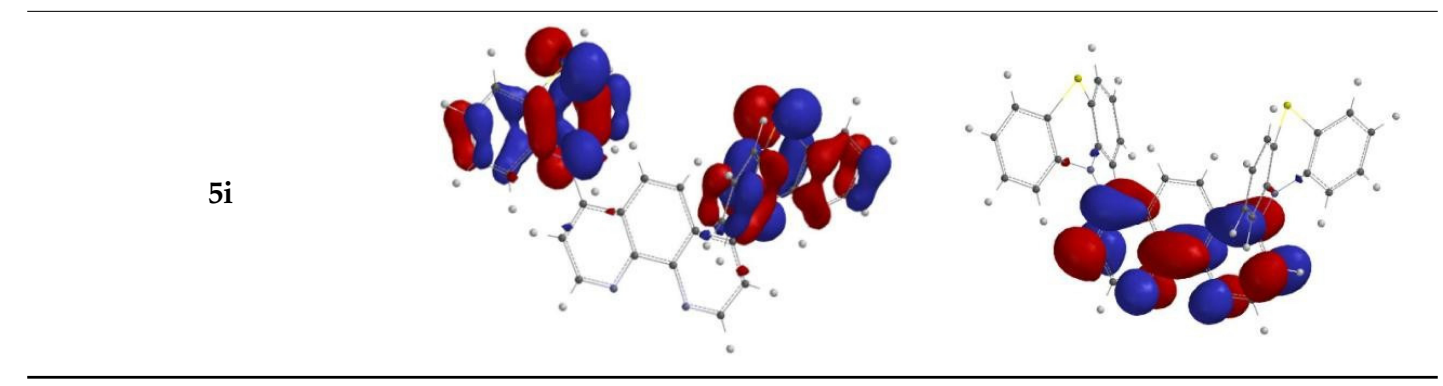


The performed investigations on the X-ray studies were inspired by the work of G. Zucchi et al. and K.J. Shaffer et al. $[25,28]$. Presented here compounds crystalize in the triclinic $P-1$ space group as a solvate with two $\mathrm{CHCl}_{3}$ molecules $5 \mathbf{d}$ and two THF and one methanol molecules in the case of compounds $\mathbf{6 a}$ and $\mathbf{6} \mathbf{b}$ crystalizes in monoclinic $P 2_{1} / \mathrm{c}$ space group with two THF molecules. The molecular structures are displayed as ORTEP representation in Figure 1a-c.

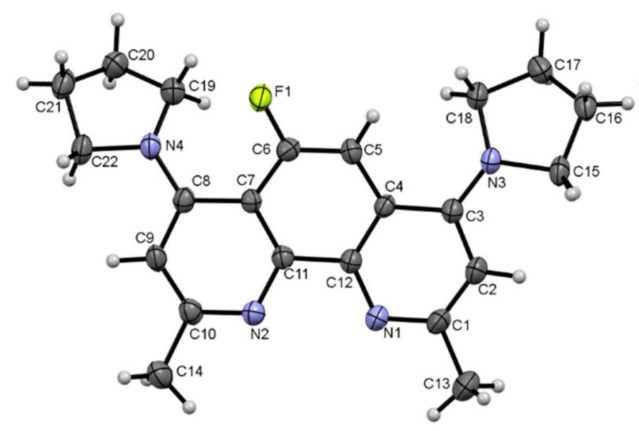

(a)

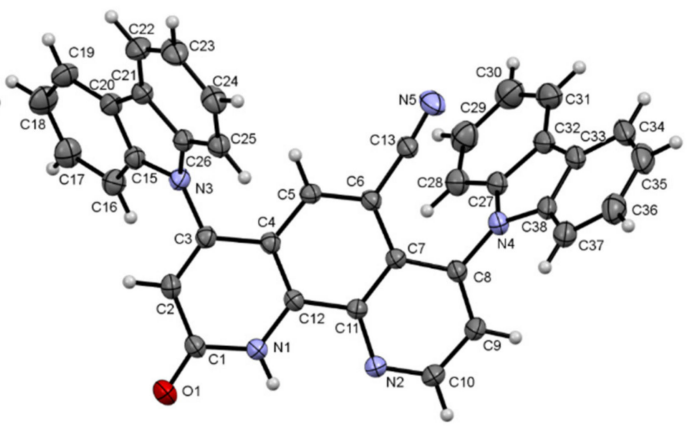

(b)

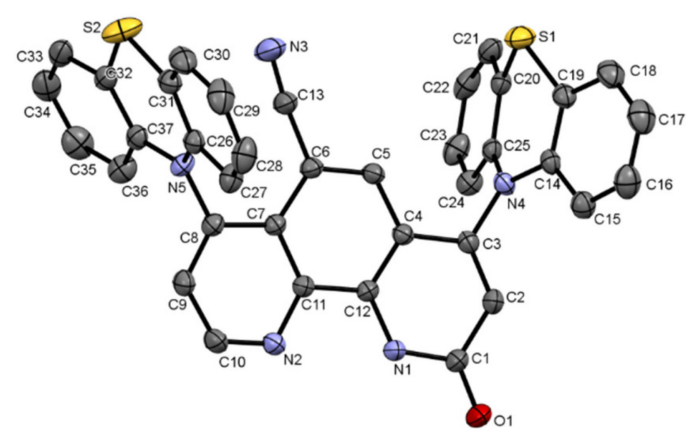

(c)

Figure 1. (a) ORTEP drawings of compound $5 \mathbf{d}$ with $30 \%$ probability. The solvent molecules and fluorine disorder were omitted for clarity. (b) ORTEP drawings of compound 6 a with $30 \%$ probability. The solvent molecules were omitted for clarity. (c) ORTEP drawings of compound $\mathbf{6 b}$ with $30 \%$ probability. Hydrogen atoms and solvent molecules are omitted for clarity.

The intra- and intermolecular hydrogen bonds in the structures of compounds $\mathbf{5 d}$ and $\mathbf{6 a}, \mathbf{6} \mathbf{b}$ are listed in Table S2 in the Supporting Information. The 1,10-pPhenanthroline moiety is in a planar orientation with a ring puckering amplitude of 0.105 in molecule $5 \mathbf{d}$ and 0.05 and 0.07 in the cases of compounds $\mathbf{6 a}$ and $\mathbf{6 b}$, respectively. In the structure of heterocycle $5 \mathbf{d} \mathrm{C}-\mathrm{Cl} \ldots \pi$ interactions are visible, while in molecules $\mathbf{6 a}$ and $\mathbf{6 b} \pi \ldots \pi$ stacking occurs between phenanthroline rings with centroid-centroid distances of $3.89 \AA$ and $3.78 \AA$ and shift distances of $1.91 \AA$ and $1.07 \AA$, respectively (Table S3; Supporting Information). The interaction between the nitrile $\mathrm{C}(13)-\mathrm{N}(3)$ group causes flattening of $10 \mathrm{H}$-phenothiazine ring in the position $\mathrm{C} 7$. The $10 \mathrm{H}$-phenothiazine ring in position $\mathrm{C} 4$ shows a characteristic, for this type of heterocyclic compounds, deviation of the sulfur atom position from the plane delimited by the benzene rings. The structures of compounds 5d, $\mathbf{6 a}$ and $\mathbf{6 b}$ are constitutionally related to 4,7-di(10H-phenoxazin-10-yl)-1,10-phenanthroline and 4,7-di(9H-carbazol-9-yl)-1,10-phenanthroline [25,28]. Similarly to two other compounds which are composed of a 1,10-phenanthroline core and two peripheral amino substituents, the $9 H$-carbazol-9-yl and $10 H$-phenoxazin-10-yl substituents are strongly twisted in relation to the 1,10-phenanthroline rings with a larger angle $\left(\sim 87^{\circ}\right)$ between 1,10-phenanthroline and substituent planes in the position C7 than $C 4\left(\sim 83^{\circ}\right)$. It is noticed that bond lengths between carbon atoms at $C 4 / 3$ and $C 7 / 8$ positions and pyrrolidinyl, 9H carbazol-9-yl and 10H-phenothiazin-10-yl groups $1.360 \AA, 1.420 \AA$ and $1.434(3) \AA$, respectively, are comparable to the values of $1.42 \AA$ in 4,7-di(9H-carbazol-9-yl)-1,10-phenanthroline or $1.43 \AA$ for 4,7-di(10H-phenoxazin-10-yl)-1,10-phenanthroline. The $\pi$-conjugated system derived from 
1,10-phenanthroline core and two peripheral $9 H$-carbazole or $10 H$-phenoxazine rings showed less extended delocalization than that of pyrrolidinyl group over the entire $\pi$-conjugated systems, because the lone pair of nitrogen atom and the 1,10-phenanthroline core are not in the same plane. $9 \mathrm{H}$-carbazole and $10 \mathrm{H}$-phenothiazine rings are perpendicular to the 1,10-phenanthroline moiety [25,28].

\subsection{NMR Studies}

${ }^{13} \mathrm{C}$ CP/MAS NMR spectra of compounds 4 and 5 displayed readily discernible aliphatic carbon signals such as methyl, and distinguishable resonance lines attributed to aromatic carbons. In general, the signals in the solution and in the solid state are in good agreement (Tables S5 and S6; Supporting Information). To investigate the character of nitrogen atoms which are responsible for their bidentate coordination abilities, studies using the ${ }^{15} \mathrm{~N} \mathrm{CP} / \mathrm{MAS}$ NMR technique were carried out, which is a highly sensitive probe and allows being monitored even in subtle changes in molecular constitution and electronic structures. Due to the symmetry of $\mathbf{4 e}, \mathbf{5 c}$ and $\mathbf{5 f}$ molecules only one or two lines are presence in ${ }^{15} \mathrm{~N} \mathrm{CP} / \mathrm{MAS}$ NMR spectrum, respectively (Table S7; Supporting Information). Comparing the experimental CP/MAS ${ }^{15} \mathrm{~N}$ chemical shifts of selected heterocyles 4 and 5, the influence of substituents originated from chlorine atoms, methyl group and $\mathrm{N}$-donor fragments i.e., $\mathrm{NH}_{2}$, pyrrolidine, $9 \mathrm{H}$-carbazole and $10 \mathrm{H}$-phenothiazine could be observed. It is worth noting that the ${ }^{15} \mathrm{~N}$ chemical shift of a free 1,10-phenanthroline (or 2,9-dimethyl-1,10-phenanthroline) molecule is equal to -74.4 (or -80.2) ppm in the absolute scale [34,35]. A significant impact on the ${ }^{15} \mathrm{~N}$ chemical shifts of pyridine nitrogen has been seen with pyrrolidine and $10 H$-phenothiazine substituents (Table S7; Supporting Information). For pyridine rings, the dominant contribution to nitrogen shielding is associated with a $n \rightarrow \pi^{*}$ excitation, and low transition energies correlate with large deshieldings. Interestingly, $9 \mathrm{H}$-carbazole and $10 \mathrm{H}$-phenothiazine fragments have higher impact on one of the two pyridine nitrogen atoms. One is significantly deshielded (downfield effect, larger $\delta$ ) with 11.14 ppm of difference for molecule 5k or 23.78 ppm of difference for compound $5 \mathbf{h}$ (Table S7). In contrast, $N$-donor substituents i.e., $\mathrm{NH}_{2}$, pyrrolidine, $9 \mathrm{H}$-carbazole and $10 \mathrm{H}$-phenothiazine are characterized by the more negative value of ${ }^{15} \mathrm{~N}$ chemical shift (-249.48 to $\left.-317.08 \mathrm{ppm}\right)$.

\subsection{Cyclic Voltammetry}

The electrochemical behaviour of selected substituted 1,10-phenanthroline derivatives in acetonitrile was studied by cyclic voltammetry on a glassy carbon electrode. Compounds 4 substituted by chlorine atoms ( $\mathbf{R}^{2}$ substituent) yield rather complex cyclic voltammograms mainly in the negative potential regions (Figure 2). In the case of all compounds 4 the first reduction wave corresponds to the formation of anion radical and according to literature the subsequent cleavage of chlorine from position $\mathbf{R}^{\mathbf{2}}$ in the overall ECE process follows [36,37]. The compounds $\mathbf{4} \mathbf{b}$ and $\mathbf{4 i}$ were found to be the most easily reduced because of the presence of chlorine and fluorine atoms as $\mathbf{R}^{\mathbf{1}}$. In the case of compound $\mathbf{4} \mathbf{i}$ the first reduction step may be attributed to the cleavage of chlorine $\left(\mathbf{R}^{\mathbf{1}}\right)$ from primarily formed radical anion. Calculated HOMO and LUMO spatial distributions of sterically symmetrical compounds are shown in Table 1 and results for other electrochemically investigated compounds are summarized in Tables S8 and S9 in the Supporting Information. A linear relationship of LUMO energy on reduction potential was observed for compounds from series 4 and 5 (except compounds $\mathbf{5 a}$ and $\mathbf{5 c}$ ) showing that the first reduction step proceeds on 1,10-phenanthroline rings and an anion radical is formed (Figure 3). Compounds $\mathbf{5 a}$ and $\mathbf{5} \mathbf{c}$ are substituted with pyrrolidine and their reduction may follow another mechanism. These two compounds differ from the others also by high values of their dipole moments as indicated in Table 2. The substituent effect on the reduction and oxidation potentials of selected compounds was summarized in Table 2. Methyl group as $\mathbf{R}$ substituent significantly influenced reduction potential of compounds towards more negative values (compounds $4 \mathbf{g}, 4 \mathbf{k}, 5 \mathrm{c}$ compare to $\mathbf{4 a}, \mathbf{4 d}, \mathbf{5 a}$, respectively). The oxidation of all compounds 4 may lead to the formation of cation radicals [38]. The dependences of the highest occupied molecular orbital energy on the first oxidation potential were linear for both series of compounds, substituents $\mathbf{R}^{2}$ with chlorine atoms 4 
and $N$-heterocycles 5. Their independence is related to their different oxidation mechanism, resulting from the different electroactive site visible from the spatial distribution of HOMO orbitals (Table 1). Oxidation of phenothiazine is known in the literature $[39,40]$. Detailed investigation of reduction and oxidation mechanism of both series will be further plan of our research. Figure 3C shows a linear relationship between the energy difference of HOMO and LUMO orbitals and the potential gap $\Delta E$ obtained experimentally.

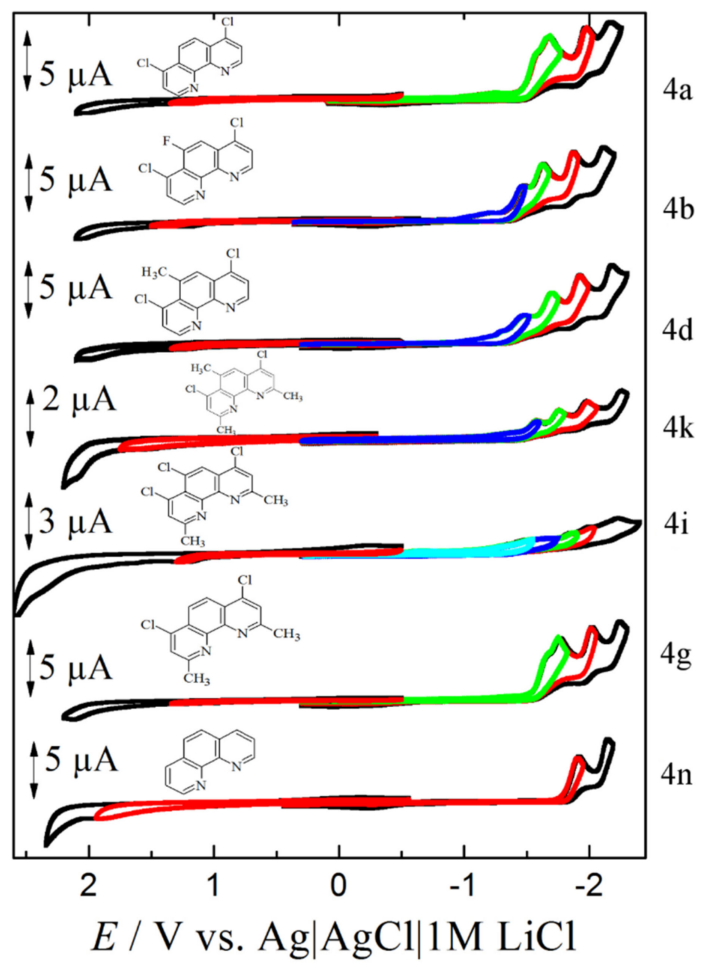

Figure 2. Cyclic voltammograms of $0.60 \mathrm{mM} 4 \mathbf{a}, \mathbf{4 g}, \mathbf{4 i}, \mathbf{4 n}$ and $0.50 \mathrm{mM} \mathbf{4 b}, \mathbf{4 d}$ and $0.12 \mathrm{mM} 4 \mathbf{k}$ in acetonitrile at $0.1 \mathrm{~V} / \mathrm{s}\left(0.1 \mathrm{M} \mathrm{TBAPF}_{6}\right)$.
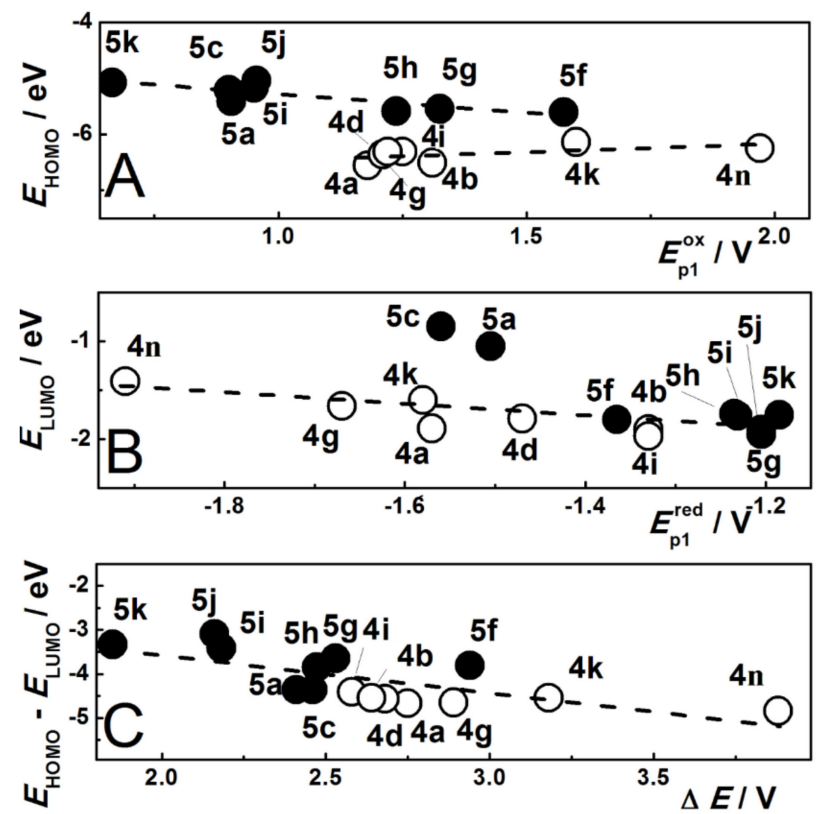

Figure 3. Dependence of HOMO (A) and LUMO (B) energies on the experimental oxidation and reduction potentials, respectively. Panel (C) shows a dependence of HOMO and LUMO energies difference on electrochemical gap, $E_{\mathrm{ox}}$ and $E_{\text {red }}$. 
Table 2. Experimentally obtained electrochemical data (the first oxidation $E_{\mathrm{p} 1}{ }^{\text {ox }}$ and the first reduction potential $E_{\mathrm{p} 1}{ }^{\text {red }}$ ) at glassy carbon electrode, and calculated energies of HOMO and LUMO by DFT (B3LYP/6-31G*).

\begin{tabular}{|c|c|c|c|c|c|}
\hline No. & $E_{\mathrm{p} 1}{ }^{o x} / \mathrm{V}$ & $E_{\mathrm{p} 1}{ }^{\mathrm{red}} / \mathrm{V}$ & $\begin{array}{l}\text { Dipole } \mu \\
\text { (calc.)/D }\end{array}$ & $E^{\mathrm{HOMO}} / \mathrm{eV}$ & $E^{\mathrm{LUMO}} / \mathrm{eV}$ \\
\hline $4 a$ & 1.181 & -1.572 & 0.74 & -6.56 & -1.89 \\
\hline $4 d$ & 1.212 & -1.471 & 1.12 & -6.36 & -1.79 \\
\hline $4 g$ & 1.221 & -1.670 & 0.47 & -6.31 & -1.66 \\
\hline $4 \mathrm{i}$ & 1.253 & -1.330 & 2.14 & -6.31 & -1.90 \\
\hline $4 b$ & 1.312 & -1.331 & 0.46 & -6.51 & -1.97 \\
\hline $4 k$ & 1.601 & -1.582 & 0.24 & -6.14 & -1.60 \\
\hline $4 n$ & 1.971 & -1.911 & 3.23 & -6.25 & -1.41 \\
\hline $5 k$ & 0.668 & -1.185 & 0.39 & -5.08 & -1.75 \\
\hline $5 c$ & 0.900 & -1.560 & 4.37 & -5.21 & -0.85 \\
\hline $5 a$ & 0.905 & -1.505 & 5.38 & -5.41 & -1.05 \\
\hline $5 i$ & 0.951 & -1.231 & 0.47 & -5.17 & -1.76 \\
\hline $5 \mathbf{j}$ & 0.956 & -1.205 & 2.47 & -5.04 & -1.95 \\
\hline $5 h$ & 1.238 & -1.235 & 1.53 & -5.59 & -1.74 \\
\hline $5 g$ & 1.325 & -1.205 & 0.61 & -5.55 & -1.90 \\
\hline $5 f$ & 1.575 & -1.365 & 1.42 & -5.61 & -1.80 \\
\hline
\end{tabular}

\section{Materials and Methods}

\subsection{Materials}

All experiments were carried out in an atmosphere of dry argon and flasks were flame dried. Solvents were dried by usual methods (diphenyl ether, diethyl ether and THF over benzophenone ketyl, $\mathrm{CHCl}_{3}$ and $\mathrm{CH}_{2} \mathrm{Cl}_{2}$ over $\mathrm{P}_{4} \mathrm{O}_{10}$, hexane over sodium-potassium alloy) and distilled. Chromatographic purification was carried out on silica gel 60 (0.15-0.3 mm, Macherey-Nagel GmbH \& Co. KG, Düren, Germany). Sodium hydride (dry, 95\%), trimethyl orthoformate, triethyl orthoformate, triethyl orthoacetate, trimethyl orthoacetate, 1,10-phenanthroline (4n), 1,10-phenanthrolin-5-amine (51), 9H-carbazole, pyrrolidine, 10H-phenothiazine, Meldrum's acid, benzene-1,2-diamine, 4-fluorobenzene-1,2-diamine, 4-chlorobenzene-1,2-diamine, 4-bromobenzene-1,2-diamine, 4-methylbenzene-1,2-diamine, 3,4-diaminobenzonitrile and 3,4-diaminobenzoic acid were purchased from Sigma-Aldrich (Poznan, Poland), and were used without further purification.

\subsection{Instrumentation}

NMR spectra were obtained with Avance 400, 500 and 600 spectrometers (Bruker, Billerica, MA, USA) operating at $600.2,500.2$ or $400.1 \mathrm{MHz}\left({ }^{1} \mathrm{H}\right), 150,125.78$ or $100.5 \mathrm{MHz}\left({ }^{13} \mathrm{C}\right)$ and $470.5 \mathrm{MHz}\left({ }^{19} \mathrm{~F}\right)$ at $21^{\circ} \mathrm{C}$, and a Bruker Avance 400WB; $100.65 \mathrm{MHz}$ for ${ }^{13} \mathrm{C}, 40.55 \mathrm{MHz}$ for ${ }^{15} \mathrm{~N}$. Chemical shifts referenced to ext. TMS $\left({ }^{1} \mathrm{H},{ }^{13} \mathrm{C}\right), \mathrm{CFCl}_{3}\left({ }^{19} \mathrm{~F}\right)$ and ext. DSS $\left({ }^{1} \mathrm{H},{ }^{13} \mathrm{C}\right)$, or using the residual $\mathrm{CHCl}_{3}$ signal $\left(\delta_{\mathrm{H}} 7.26\right.$ ppm) and $\mathrm{CDCl}_{3}\left(\delta_{\mathrm{C}} 77.1 \mathrm{ppm}\right)$ as internal references for ${ }^{1} \mathrm{H}$ and ${ }^{13} \mathrm{C} \mathrm{NMR}$, respectively. Coupling constants are given in Hz. High-resolution solid-state Cross-Polarization $(\mathrm{CP}){ }^{13} \mathrm{C}$ - and ${ }^{15} \mathrm{~N}-\mathrm{NMR}$ experiments were made with magic-angle spinning (MAS) at 5 and $7 \mathrm{kHz}$. Experiments were done with a 4000 scans, a contact time of $2 \mathrm{~ms}$, a repetition time of $25 \mathrm{~s}$, using 4 and $7 \mathrm{~mm}$ Bruker probes. For GC-MS a 7890A gas chromatograph (Agilent Technologies, Wilmington, DE, USA) equipped with a MS (70 eV) 5975 EI/CI MSD, and a 7693 autosampler with an Agilent HP-5MS capillary column $(30 \mathrm{~m} \times 250 \mu \mathrm{m} \times 0.25 \mu \mathrm{m})$-press. $127.5 \mathrm{kPa}$, total flow $19 \mathrm{~mL} / \mathrm{min}$, col. flow $2 \mathrm{~mL} / \mathrm{min}$, split - 7:1, temp. prog. $\left(70^{\circ} \mathrm{C}\right.$ - hold $0.5 \mathrm{~min}, 70-290^{\circ} \mathrm{C} / 25^{\circ} \mathrm{C} / \mathrm{min}$., $290^{\circ} \mathrm{C}$ - hold $6 \mathrm{~min}$ ) was used. The LCMS-IT-TOF analysis was performed on an Agilent 1200 Series binary LC system coupled to a micrOTOF-Q system mass spectrometer (Bruker Daltonics, Brema, Germany). High-resolution mass spectrometry (HRMS) measurements were performed using a Synapt G2-Si mass spectrometer (Waters, New Castle, DE USA) equipped with an ESI source and quadrupole-time-of-flight mass analyser. To ensure accurate mass 
measurements, data were collected in centroid mode and mass was corrected during acquisition using leucine enkephalin solution as an external reference (Lock-Spray ${ }^{\mathrm{TM}}$ ). The results of the measurements were processed using the MassLynx 4.1 software (Waters) incorporated within the instrument. A Nicolet iS50 FTIR spectrometer was used for recording spectra in the IR range $4000-400 \mathrm{~cm}^{-1}$. FTIR spectra were recorded on a Perkin Elmer (Schwerzenbach, Switzerland) spectrophotometer in the spectral range $4000-450 \mathrm{~cm}^{-1}$ with the samples in the form of $\mathrm{KBr}$ pellets. Elementary analysis was performed using Vario EL III apparatus (Elementar, Langenselbold, Germany). Melting points were determined on MPA100 OptiMelt melting point apparatus (Stanford Research Systems, Sunnyvale, CA USA) and are uncorrected.

\subsection{General Procedures for Synthesis of 4,7-dichloro-1,10-phenanthrolines}

These syntheses were based on procedures described in the literature [22,23].

\subsubsection{Step A}

Triethyl orthoformate or trimethyl orthoformate were used in the synthesis of $\mathbf{2 a}, \mathbf{2} \mathbf{b}, \mathbf{2 c}, \mathbf{2 d}$, $\mathbf{2 e}, 2 \mathbf{f}$ and $2 \mathrm{~g}$ and triethyl orthoacetate or trimethyl orthoacetate for $2 \mathbf{h}, \mathbf{2 i}, \mathbf{2 j}, \mathbf{2 k}, \mathbf{2}, \mathbf{2} \mathbf{m}, \mathbf{2 n}$ and 2o. The orthoester (triethyl orthoformate, triethyl orthoacetate, trimethyl orthoformate or trimethyl orthoacetate, $330.0 \mathrm{mmol}$ ) and Meldrum's acid $(1.7 \mathrm{~g}, 12.0 \mathrm{mmol})$ was brought to a gentle reflux for 15 min. The resulting greenish solution was cooled to $80^{\circ} \mathrm{C}$ and the appropriate benzene-1,2-diamine 1 ( $5.5 \mathrm{mmol}$ ) was added portionwise (caution: exothermic reaction). The resulting mixture was stirring up to reflux for $2 \mathrm{~h}$, and left under r.t. for $16 \mathrm{~h}$. Subsequently, diethyl ether or hexane was added and the solution was cooled to $-35^{\circ} \mathrm{C}$ where a precipitate formed. The precipitate was filtered off, washed with diethyl ether $(4 \times 100 \mathrm{~mL})$ and dried to afford a solid:

1,2-Bis-[(2,2-dimethyl-4,6-dioxo-1,3-dioxan-5-ylidenemethyl)amino]benzene (2a) [23,41]; white solid $2.0 \mathrm{~g}$ $(4.7 \mathrm{mmol}, 86 \%) ; \mathrm{m} . \mathrm{p}_{\mathrm{dec}} \cdot=207.6^{\circ} \mathrm{C}$.

5,5'-((4-Fluoro-1,2-phenylene)bis(azanediyl))bis(methaneylylidene))bis(2,2-dimethyl-1,3-dioxane-4,6-dione) (2b); white; $2.0 \mathrm{~g}(4.6 \mathrm{mmol}, 83 \%)$; m.p $\mathrm{pdec}=216{ }^{\circ} \mathrm{C} ;{ }^{1} \mathrm{H}-\mathrm{NMR}\left(\mathrm{CDCl}_{3} ; 400.2 \mathrm{MHz}\right) \delta=1.73(\mathrm{~s}, 6 \mathrm{H}$, $\left.2 \mathrm{CH}_{3}\right), 1.74\left(\mathrm{~s}, 6 \mathrm{H}, 2 \mathrm{CH}_{3}\right), 7.06-7.12\left(\mathrm{dt}^{3}{ }^{3} \mathrm{~J}_{\mathrm{H}, \mathrm{H}}=8.1 \mathrm{~Hz},{ }^{4} J_{\mathrm{F}, \mathrm{H}}=2.6 \mathrm{~Hz}, 1 \mathrm{H}\right.$, aromatic), 7.20 (dd, ${ }^{3} J_{\mathrm{F}, \mathrm{H}}=8.7 \mathrm{~Hz},{ }^{4} J_{\mathrm{H}, \mathrm{H}}=2.5 \mathrm{~Hz}, 1 \mathrm{H}$, aromatic), $7.38\left(\mathrm{dd},{ }^{3} J_{\mathrm{F}, \mathrm{H}}=8.9 \mathrm{~Hz},{ }^{3} J_{\mathrm{H}, \mathrm{H}}=5.1 \mathrm{~Hz}\right.$, aromatic), $8.38(\mathrm{~d}$, ${ }^{3} J_{\mathrm{H}, \mathrm{H}}=13.5 \mathrm{~Hz}, 1 \mathrm{H}$, vinyl), $8.52\left(\mathrm{~d},{ }^{3} J_{\mathrm{H}, \mathrm{H}}=13.4 \mathrm{~Hz}, 1 \mathrm{H}\right.$, vinyl $), 11.15\left(\mathrm{~d},{ }^{3} J_{\mathrm{H}, \mathrm{H}}=13.5 \mathrm{~Hz}, 1 \mathrm{H}, \mathrm{NH}\right)$, $11.42\left(\mathrm{~d},{ }^{3} J_{\mathrm{H}, \mathrm{H}}=13.3 \mathrm{~Hz}, 1 \mathrm{H}, \mathrm{NH}\right) ;{ }^{13} \mathrm{C}\left\{{ }^{1} \mathrm{H}\right\}-\mathrm{NMR}\left(\mathrm{CDCl}_{3} ; 125.78 \mathrm{MHz}\right) \delta=27.2,27.3,89.6,90.3,105.8$ $\left(\mathrm{d},{ }^{3} J_{\mathrm{C}, \mathrm{F}}=14.3 \mathrm{~Hz}\right), 107.4\left(\mathrm{~d},{ }^{2} J_{\mathrm{C}, \mathrm{F}}=26.8 \mathrm{~Hz}\right), 115.0\left(\mathrm{~d},{ }^{2} J_{\mathrm{C}, \mathrm{F}}=22.9 \mathrm{~Hz}\right), 124.9\left(\mathrm{~d},{ }^{4} J_{\mathrm{C}, \mathrm{F}}=9.5 \mathrm{~Hz}\right), 126.9$, $133.3\left(\mathrm{~d},{ }^{3} J_{\mathrm{C}, \mathrm{F}}=10.1 \mathrm{~Hz}\right), 161.1,155.1\left(\mathrm{~d},{ }^{1} J_{\mathrm{C}, \mathrm{F}}=342.9 \mathrm{~Hz}\right), 162.9,163.2,165.4,165.5 ;{ }^{19} \mathrm{~F}\left\{{ }^{1} \mathrm{H}\right\}-\mathrm{NMR}$ $\left(\mathrm{CDCl}_{3} ; 470.5 \mathrm{MHz}\right) \delta=-108.94 ;{ }^{19} \mathrm{~F}-\mathrm{NMR}\left(\mathrm{CDCl}_{3} ; 470.5 \mathrm{MHz}\right) \delta=-108.94\left(\mathrm{dd},{ }^{3} J_{\mathrm{F}, \mathrm{H}}=12.9 \mathrm{~Hz}\right.$, ${ }^{3} J_{\mathrm{F}, \mathrm{H}}=7.8 \mathrm{~Hz}$ ); UV-Vis (methanol; $\lambda$ [nm] (loge)): 329 (4.48), 292 (4.56), 212 (4.43).

5,5'-((4-Chloro-1,2-phenylene)bis(azanediyl))bis(methaneylylidene))bis(2,2-dimethyl-1,3-dioxane-4,6-dione) (2c) [23]; white; $2.1 \mathrm{~g}(4.6 \mathrm{mmol}, 84 \%) ; \mathrm{m} . \mathrm{p}_{\mathrm{dec}}=202.7^{\circ} \mathrm{C}$; UV-Vis (methanol; $\left.\lambda[\mathrm{nm}](\log \varepsilon)\right): 332(4.59)$, 296 (4.75), 223 (4.47), 206 (4.46).

5,5'-(((4-Methyl-1,2-phenylene)bis(azanediyl))bis(methaneylylidene))bis(2,2-dimethyl-1,3-dioxane-4,6-dione) (2d); white; $2.2 \mathrm{~g}(5.2 \mathrm{mmol}, 94 \%)$ [41]; m.p $\mathrm{pec}=212{ }^{\circ} \mathrm{C}$; UV-Vis (methanol; $\left.\lambda[\mathrm{nm}](\log \varepsilon)\right): 330$ (4.85), 296 (4.93), 221 (4.83).

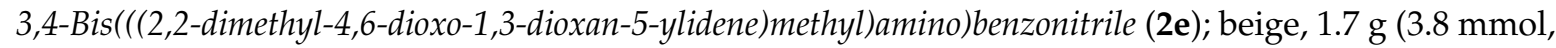
$69 \%) ;$ m.p dec $=250{ }^{\circ} \mathrm{C} ;{ }^{1} \mathrm{H}-\mathrm{NMR}(\mathrm{DMSO}-d 6 ; 400.2 \mathrm{MHz}) \delta=1.68\left(\mathrm{~s}, 6 \mathrm{H}, 2 \mathrm{CH}_{3}\right), 1.68\left(\mathrm{~s}, 6 \mathrm{H}, 2 \mathrm{CH}_{3}\right)$, 7.81-7.84 (m, 2H, aromatic), $8.16\left(\mathrm{dd}^{3}{ }^{3} \mathrm{~J}_{\mathrm{H}, \mathrm{H}}=4.3 \mathrm{~Hz},{ }^{4} J_{\mathrm{H}, \mathrm{H}}=0.8 \mathrm{~Hz}, 1 \mathrm{H}\right.$, aromatic), $8.33\left(\mathrm{~d},{ }^{3} J_{\mathrm{H}, \mathrm{H}}=14.1\right.$ $\mathrm{Hz}, 1 \mathrm{H}$, vinyl), $8.41\left(\mathrm{~d},{ }^{3} J_{\mathrm{H}, \mathrm{H}}=13.9 \mathrm{~Hz}, 1 \mathrm{H}\right.$, vinyl), $11.33\left(\mathrm{~d},{ }^{3} J_{\mathrm{H}, \mathrm{H}}=14.1 \mathrm{~Hz}, 1 \mathrm{H}, \mathrm{NH}\right), 11.43(\mathrm{~d}$, $\left.{ }^{3} J_{\mathrm{H}, \mathrm{H}}=14.0 \mathrm{~Hz}, 1 \mathrm{H}, \mathrm{NH}\right) ;{ }^{13} \mathrm{C}\left\{{ }^{1} \mathrm{H}\right\}-\mathrm{NMR}$ (DMSO-d6; $\left.125.78 \mathrm{MHz}\right) \delta=26.5,26.6,88.3,88.8,104.1,104.3$, 110.0, 117.4, 122.7, 128.0, 132.0, 133.6, 137.5, 155.6, 157.4, 162.3, 162.5, 163.5, 163.6; UV-Vis (methanol; $\lambda$ [nm] (loge)): 342 (4.11), $296(4.19), 219$ (4.00). 
3,4-Bis(((2,2-dimethyl-4,6-dioxo-1,3-dioxan-5-ylidene)methyl)amino)benzoic acid (2f); beige, $0.8 \mathrm{~g}$ (1.8 mmol, $32 \%)$; m.p $\mathrm{dec}=22{ }^{\circ} \mathrm{C} ;{ }^{1} \mathrm{H} \mathrm{NMR}\left(\mathrm{CDCl}_{3} ; 400.2 \mathrm{MHz}\right) \delta=1.77\left(\mathrm{~s}, 6 \mathrm{H}, 2 \mathrm{CH}_{3}\right), 1.79\left(\mathrm{~s}, 6 \mathrm{H}, 2 \mathrm{CH}_{3}\right), 7.50$ $\left(\mathrm{d},{ }^{3} J_{\mathrm{H}, \mathrm{H}}=8.5 \mathrm{~Hz}, 1 \mathrm{H}\right.$, aromatic $), 8.14\left(\mathrm{~d},{ }^{3} J_{\mathrm{H}, \mathrm{H}}=8.9 \mathrm{~Hz}, 1 \mathrm{H}\right.$, aromatic $), 8.50(\mathrm{~s}, 1 \mathrm{H}$, aromatic), $8.59(\mathrm{~d}$, ${ }^{3} J_{\mathrm{H}, \mathrm{H}}=13.1 \mathrm{~Hz}, 1 \mathrm{H}$, vinyl), $8.99\left(\mathrm{~d},{ }^{3} \mathrm{~J}_{\mathrm{H}, \mathrm{H}}=12.7 \mathrm{~Hz}, 1 \mathrm{H}\right.$, vinyl), $11.51\left(\mathrm{~d},{ }^{3} \mathrm{~J}_{\mathrm{H}, \mathrm{H}}=12.6 \mathrm{~Hz}, 1 \mathrm{H}, \mathrm{NH}\right)$, $11.66\left(\mathrm{~d},{ }^{3} \mathrm{~J}_{\mathrm{H}, \mathrm{H}}=12.9 \mathrm{~Hz}, 1 \mathrm{H}, \mathrm{NH}\right){ }^{1} \mathrm{H}-\mathrm{NMR}(\mathrm{DMSO}-d 6 ; 500.2 \mathrm{MHz}) \delta=1.68\left(\mathrm{~s}, 6 \mathrm{H}, 2 \mathrm{CH}_{3}\right), 1.68(\mathrm{~s}, 6 \mathrm{H}$, $\left.2 \mathrm{CH}_{3}\right), 7.74\left(\mathrm{~d},{ }^{3} J_{\mathrm{H}, \mathrm{H}}=8.4 \mathrm{~Hz}, 1 \mathrm{H}\right.$, aromatic $), 7.96\left(\mathrm{dd},{ }^{3} J_{\mathrm{H}, \mathrm{H}}=8.3 \mathrm{~Hz},{ }^{4} J_{\mathrm{H}, \mathrm{H}}=1.7 \mathrm{~Hz}, 1 \mathrm{H}\right.$, aromatic), $8.03\left(\mathrm{~d},{ }^{4} J_{\mathrm{H}, \mathrm{H}}=1.7 \mathrm{~Hz}, 1 \mathrm{H}\right.$, aromatic $), 8.24\left(\mathrm{~d},{ }^{3} J_{\mathrm{H}, \mathrm{H}}=14.2 \mathrm{~Hz}, 1 \mathrm{H}\right.$, vinyl $), 8.40\left(\mathrm{~d},{ }^{3} J_{\mathrm{H}, \mathrm{H}}=14.1 \mathrm{~Hz}, 1 \mathrm{H}\right.$, vinyl), $11.35\left(\mathrm{~d},{ }^{3} \mathrm{~J}_{\mathrm{H}, \mathrm{H}}=14.3 \mathrm{~Hz}, 1 \mathrm{H}, \mathrm{NH}\right), 11.44\left(\mathrm{~d},{ }^{3} \mathrm{~J}_{\mathrm{H}, \mathrm{H}}=14.1 \mathrm{~Hz}, 1 \mathrm{H}, \mathrm{NH}\right) ;{ }^{13} \mathrm{C}\left\{{ }^{1} \mathrm{H}\right\}-\mathrm{NMR}$ (DMSO-d6; $125.78 \mathrm{MHz}) \delta=26.50,26.52,87.8,88.2,104.0,104.1,121.9,125.1,129.3,130.0,133.1,137.2,155.7,157.5$, 162.4, 162.7, 163.4, 163.6, 165.9, 168.2; UV-Vis (methanol; $\lambda$ [nm] (loge)): 340 (3.96),303 (3.87) 274 (3.82), 220 (4.00).

Methyl 3,4-bis(((2,2-dimethyl-4,6-dioxo-1,3-dioxan-5-ylidene)methyl)amino)benzoate (2g); beige, $1.6 \mathrm{~g}$ $(3.5 \mathrm{mmol}, 63 \%)$; m.p. $\mathrm{dec}=225^{\circ} \mathrm{C} ;{ }^{1} \mathrm{H}-\mathrm{NMR}(\mathrm{DMSO}-d 6 ; 500.2 \mathrm{MHz}) \delta=1.68\left(\mathrm{~s}, 6 \mathrm{H}, 2 \mathrm{CH}_{3}\right), 1.69(\mathrm{~s}, 6 \mathrm{H}$, $\left.2 \mathrm{CH}_{3}\right), 4.00\left(\mathrm{~s}, 3 \mathrm{H}, \mathrm{OCH}_{3}\right), 7.39\left(\mathrm{~d},{ }^{3} J_{\mathrm{H}, \mathrm{H}}=8.3 \mathrm{~Hz}, 1 \mathrm{H}\right.$, aromatic $), 7.76\left(\mathrm{dd},{ }^{3} J_{\mathrm{H}, \mathrm{H}}=8.2 \mathrm{~Hz},{ }^{4} J_{\mathrm{H}, \mathrm{H}}=1.7\right.$ $\mathrm{Hz}, 1 \mathrm{H}$, aromatic), $8.18\left(\mathrm{~d},{ }^{4} J_{\mathrm{H}, \mathrm{H}}=1.6 \mathrm{~Hz}, 1 \mathrm{H}\right.$, aromatic $), 8.24\left(\mathrm{~d},{ }^{3} J_{\mathrm{H}, \mathrm{H}}=14.5 \mathrm{~Hz}, 1 \mathrm{H}\right.$, vinyl), $8.81(\mathrm{~d}$, ${ }^{3} J_{\mathrm{H}, \mathrm{H}}=14.7 \mathrm{~Hz}, 1 \mathrm{H}$, vinyl), $11.40\left(\mathrm{~d},{ }^{3} \mathrm{~J}_{\mathrm{H}, \mathrm{H}}=14.1 \mathrm{~Hz}, 2 \mathrm{H}, \mathrm{NH}\right), 11.84\left(\mathrm{~d},{ }^{3} \mathrm{~J}_{\mathrm{H}, \mathrm{H}}=14.8 \mathrm{~Hz}, 2 \mathrm{H}, \mathrm{NH}\right)$; ${ }^{13} \mathrm{C}\left\{{ }^{1} \mathrm{H}\right\}-\mathrm{NMR}$ (DMSO-d6; $\left.125.78 \mathrm{MHz}\right) \delta=26.47,26.52,54.2,87.2,87.7,104.39,104.38,116.0,119.9$, 127.2, 128.0, 131.3, 140.1, 151.3, 159.3, 162.5, 164.7, 166.6, 172.6; UV-Vis (methanol; $\lambda$ [nm] $(\log \varepsilon)): 340$ (3.96),300 (3.87) 275 (3.82), 220 (4.00).

5,5'-((1,2-Phenylenebis(azanediyl))bis(ethan-1-yl-1-ylidene))bis(2,2-dimethyl-1,3-dioxane-4,6-dione) (2h) [22,23]; white; $1.2 \mathrm{~g}(2.8 \mathrm{mmol}, 51 \%) ; \mathrm{m} . \mathrm{p}_{\mathrm{dec}} .=189.6{ }^{\circ} \mathrm{C}$.

5,5'-(((4-Fluoro-1,2-phenylene)bis(azanediyl))bis(ethan-1-yl-1-ylidene))bis(2,2-dimethyl-1,3-dioxane-4,6-dione) (2i); white, $0.9 \mathrm{~g}(2.0 \mathrm{mmol}, 36 \%)$; m.p dec. $=218.0{ }^{\circ} \mathrm{C} ;{ }^{1} \mathrm{H}-\mathrm{NMR}\left(\mathrm{CDCl}_{3} ; 400.2 \mathrm{MHz}\right) \delta=1.70(\mathrm{~s}, 6 \mathrm{H}$, $\left.2 \mathrm{CH}_{3}\right), 1.71\left(\mathrm{~s}, 6 \mathrm{H}, 2 \mathrm{CH}_{3}\right), 2.50\left(\mathrm{~s}, 3 \mathrm{H}, \mathrm{CH}_{3}\right), 2.58\left(\mathrm{~s}, 3 \mathrm{H}, \mathrm{CH}_{3}\right), 7.12\left(\mathrm{dd},{ }^{3} J_{\mathrm{H}, \mathrm{F}}=8.2 \mathrm{~Hz},{ }^{4} J_{\mathrm{H}, \mathrm{H}}=2.3\right.$ $\mathrm{Hz}, 1 \mathrm{H}$, aromatic), 7.18-7.20 (m, 1H, aromatic), $7.35\left(\mathrm{dd},{ }^{3} J_{\mathrm{H}, \mathrm{F}}=8.7 \mathrm{~Hz},{ }^{3} \mathrm{~J}_{\mathrm{H}, \mathrm{H}}=5.4 \mathrm{~Hz}, 1 \mathrm{H}\right.$, aromatic), 12.68 (s, 1H, NH), 12.85 (s, 1H, NH); ${ }^{13} \mathrm{C}\left\{{ }^{1} \mathrm{H}\right\}-\mathrm{NMR}\left(\mathrm{CDCl}_{3} ; 125.78 \mathrm{MHz}\right) \delta=19.6,19.7,26.7,26.7,87.6$, 88.0, 103.4, 103.5, $115.4\left(\mathrm{~d},{ }^{2} J_{\mathrm{C}, \mathrm{F}}=24.8 \mathrm{~Hz}\right), 116.4\left(\mathrm{~d},{ }^{2} J_{\mathrm{C}, \mathrm{F}}=22.4 \mathrm{~Hz}\right), 128.5\left(\mathrm{~d},{ }^{4} J_{\mathrm{C}, \mathrm{F}}=3.7 \mathrm{~Hz}\right), 129.7(\mathrm{~d}$, $\left.{ }^{3} J_{\mathrm{C}, \mathrm{F}}=9.5 \mathrm{~Hz}\right), 134.1\left(\mathrm{~d},{ }^{3} J_{\mathrm{C}, \mathrm{F}}=10.3 \mathrm{~Hz}\right), 161.8\left(\mathrm{~d},{ }^{1} J_{\mathrm{C}, \mathrm{F}}=252.9 \mathrm{~Hz}\right), 162.3,162.5,167.7,167.6,172.8$, $173.5 ;{ }^{19} \mathrm{~F}\left\{{ }^{1} \mathrm{H}\right\}-\mathrm{NMR}\left(\mathrm{CDCl}_{3} ; 470.5 \mathrm{MHz}\right) \delta=-108.53 ;{ }^{19} \mathrm{~F}-\mathrm{NMR}\left(\mathrm{CDCl}_{3} ; 470.5 \mathrm{MHz}\right) \delta=-108.53(\mathrm{dd}$, $\left.{ }^{3} J_{\mathrm{F}, \mathrm{H}}=13.3 \mathrm{~Hz},{ }^{4} J_{\mathrm{F}, \mathrm{H}}=7.7 \mathrm{~Hz}\right) ; \mathrm{UV}-\mathrm{Vis}($ methanol; $\lambda[\mathrm{nm}](\log \varepsilon)): 311(4.30), 283(4.66), 226(4.38), 201$ (4.27).

5,5'-(((4-Chloro-1,2-phenylene)bis(azanediyl))bis(ethan-1-yl-1-ylidene))bis(2,2-dimethyl-1,3-dioxane-4,6-dione) (2j); white, $1.4 \mathrm{~g}(2.9 \mathrm{mmol}, 52 \%)$; $\mathrm{mp}_{\text {dec. }}=203.8{ }^{\circ} \mathrm{C} ;{ }^{1} \mathrm{H}-\mathrm{NMR}\left(\mathrm{CDCl}_{3} ; 400.2 \mathrm{MHz}\right) \delta=1.71(\mathrm{~s}, 12 \mathrm{H}$, $\left.4 \mathrm{CH}_{3}\right), 2.53\left(\mathrm{~s}, 3 \mathrm{H}, \mathrm{CH}_{3}\right), 2.56\left(\mathrm{~s}, 3 \mathrm{H}, \mathrm{CH}_{3}\right), 7.30\left(\mathrm{~d},{ }^{3} J_{\mathrm{H}, \mathrm{H}}=8.5 \mathrm{~Hz}, 1 \mathrm{H}\right.$, aromatic), $7.38\left(\mathrm{~d},{ }^{4} J_{\mathrm{H}, \mathrm{H}}=1.6\right.$ $\mathrm{Hz}, 1 \mathrm{H}$, aromatic), $7.48\left(\mathrm{dd},{ }^{3} J_{\mathrm{H}, \mathrm{H}}=8.5 \mathrm{~Hz},{ }^{4} J_{\mathrm{H}, \mathrm{H}}=1.8 \mathrm{~Hz}, 1 \mathrm{H}\right.$, aromatic), $12.76(\mathrm{~s}, 1 \mathrm{H}, \mathrm{NH}), 12.81$ (s, $1 \mathrm{H}, \mathrm{NH}) ;{ }^{13} \mathrm{C}\left\{{ }^{1} \mathrm{H}\right\}-\mathrm{NMR}\left(\mathrm{CDCl}_{3} ; 100.5 \mathrm{MHz}\right) \delta=19.7,19.7,26.7,87.9,88.0,103.5,103.5,128.1,129.0$, $129.5,131.1,133.6,135.0,162.3,162.4,167.7,167.7,173.0,173.1 ; \mathrm{UV}-\mathrm{Vis}$ (methanol; $\lambda$ [nm] (loge)): 315 (4.36), 285 (4.74), 226 (4.46), 202 (4.38).

5,5'-(((4-Bromo-1,2-phenylene)bis(azanediyl))bis(ethan-1-yl-1-ylidene))bis(2,2-dimethyl-1,3-dioxane-4,6-dione) (2k); white, $0.9 \mathrm{~g}(1.8 \mathrm{mmol}, 32 \%)$; m.p $\mathrm{dec}=204.6^{\circ} \mathrm{C} ;{ }^{1} \mathrm{H}-\mathrm{NMR}\left(\mathrm{CDCl}_{3} ; 400.2 \mathrm{MHz}\right) \delta=1.71(\mathrm{~s}, 12 \mathrm{H}$, $\left.4 \mathrm{CH}_{3}\right), 2.53\left(\mathrm{~s}, 3 \mathrm{H}, \mathrm{CH}_{3}\right), 2.56\left(\mathrm{~s}, 3 \mathrm{H}, \mathrm{CH}_{3}\right), 7.23\left(\mathrm{~d},{ }^{3} J_{\mathrm{H}, \mathrm{H}}=8.5 \mathrm{~Hz}, 1 \mathrm{H}\right.$, aromatic), $7.53\left(\mathrm{~d},{ }^{4} J_{\mathrm{H}, \mathrm{H}}=1.5\right.$ $\mathrm{Hz}, 1 \mathrm{H}$, aromatic), $7.63\left(\mathrm{dd},{ }^{3} J_{\mathrm{H}, \mathrm{H}}=8.5 \mathrm{~Hz},{ }^{4} J_{\mathrm{H}, \mathrm{H}}=1.7 \mathrm{~Hz}, 1 \mathrm{H}\right.$, aromatic), $12.76(\mathrm{~s}, 1 \mathrm{H}, \mathrm{NH}), 12.81$ (s, $1 \mathrm{H}, \mathrm{NH}) ;{ }^{13} \mathrm{C}\left\{{ }^{1} \mathrm{H}\right\}-\mathrm{NMR}\left(\mathrm{CDCl}_{3} ; 125.78 \mathrm{MHz}\right) \delta=19.6,19.7,26.6,87.7,87.8,103.4,103.4,122.3,129.1$, 131.0, 131.6, 132.4, 133.7, 162.2, 162.3, 167.5, 172.9; UV-Vis (methanol; $\lambda$ [nm] $(\log \varepsilon)): 313(4.29), 284$ (4.65), 226 (4.38), 201 (4.37).

5,5'-(((4-Methyl-1,2-phenylene)bis(azanediyl))bis(ethan-1-yl-1-ylidene))bis(2,2-dimethyl-1,3-dioxane-4,6-dione) (21); white, $1.3 \mathrm{~g}(2.9 \mathrm{mmol}, 52 \%)$; m.p dec $=204.2{ }^{\circ} \mathrm{C} ;{ }^{1} \mathrm{H}-\mathrm{NMR}\left(\mathrm{CDCl}_{3} ; 400.2 \mathrm{MHz}\right) \delta=1.70(\mathrm{~s}$, $\left.12 \mathrm{H}, 4 \mathrm{CH}_{3}\right), 2.45\left(\mathrm{~s}, 3 \mathrm{H}, \mathrm{CH}_{3}\right), 2.51\left(\mathrm{~s}, 3 \mathrm{H}, \mathrm{CH}_{3}\right), 2.52\left(\mathrm{~s}, 3 \mathrm{H}, \mathrm{CH}_{3}\right), 7.17(\mathrm{~s}, 1 \mathrm{H}$, aromatic), $7.23(\mathrm{~d}$, 
${ }^{3} J_{\mathrm{H}, \mathrm{H}}=8.1 \mathrm{~Hz}, 1 \mathrm{H}$, aromatic), $7.30\left(\mathrm{~d},{ }^{3} J_{\mathrm{H}, \mathrm{H}}=8.3 \mathrm{~Hz}, 1 \mathrm{H}\right.$, aromatic), $12.68(\mathrm{~s}, 1 \mathrm{H}, \mathrm{NH}), 12.71(\mathrm{~s}, 1 \mathrm{H}$, $\mathrm{NH}) ;{ }^{13} \mathrm{C}\left\{{ }^{1} \mathrm{H}\right\}-\mathrm{NMR}\left(\mathrm{CDCl}_{3} ; 125.78 \mathrm{MHz}\right) \delta=19.6,19.6,21.2,26.6,87.1,87.2,103.2,127.7,128.5,129.8$, 130.1, 132.2, 140.1, 162.5, 162.5, 167.6, 173.2, 173.4; UV-Vis (methanol; $\lambda$ [nm] (loge)): 319 (4.12), 283 (4.60), 227 (4.38), 203 (4.23).

3,4-Bis((1-(2,2-dimethyl-4,6-dioxo-1,3-dioxan-5-ylidene)ethyl)amino)benzonitrile (2m); white, $1.0 \mathrm{~g}$ (2.2 mmol, $40 \%)$; m.p dec $=205.6^{\circ} \mathrm{C}^{1}{ }^{1} \mathrm{H}-\mathrm{NMR}\left(\mathrm{CDCl}_{3} ; 400.2 \mathrm{MHz}\right) \delta=1.72\left(\mathrm{bs}, 12 \mathrm{H}, 4 \mathrm{CH}_{3}\right), 2.55\left(\mathrm{~s}, 3 \mathrm{H}, \mathrm{CH}_{3}\right), 2.61$ $\left(\mathrm{s}, 3 \mathrm{H}, \mathrm{CH}_{3}\right), 7.49\left(\mathrm{~d},{ }^{3} J_{\mathrm{H}, \mathrm{H}}=8.2 \mathrm{~Hz}, 1 \mathrm{H}\right.$, aromatic), $7.68(\mathrm{~s}, 1 \mathrm{H}$, aromatic $), 7.78\left(\mathrm{~d},{ }^{3} J_{\mathrm{H}, \mathrm{H}}=8.2 \mathrm{~Hz}, 1 \mathrm{H}\right.$, aromatic), $12.87(\mathrm{~s}, 1 \mathrm{H}, \mathrm{NH}), 13.04(\mathrm{~s}, 1 \mathrm{H}, \mathrm{NH}) ;{ }^{13} \mathrm{C}\left\{{ }^{1} \mathrm{H}\right\}-\mathrm{NMR}\left(\mathrm{CDCl}_{3} ; 125.78 \mathrm{MHz}\right) \delta=19.7,19.9,26.8$, 26.8, 88.6, 89.0, 103.7, 103.7, 112.7, 116.7, 128.2, 131.6, 132.6, 133.1, 136.9, 162.1, 162.2, 167.7, 167.7, 172.2, 172.9; UV-Vis (methanol; $\lambda[\mathrm{nm}](\log \varepsilon)): 324$ (4.27), 289 (4.58), 224 (4.40), 204 (4.40).

Methyl 3,4-bis((1-(2,2-dimethyl-4,6-dioxo-1,3-dioxan-5-ylidene)ethyl)amino)benzoate (2n); white, $1.2 \mathrm{~g}$ $(2.3 \mathrm{mmol}, 42 \%) ; \mathrm{m} . \mathrm{p}_{\text {dec }}=184.8{ }^{\circ} \mathrm{C} ;{ }^{1} \mathrm{H}-\mathrm{NMR}\left(\mathrm{CDCl}_{3} ; 400.2 \mathrm{MHz}\right) \delta=1.72\left(\mathrm{~s}, 6 \mathrm{H}, 2 \mathrm{CH}_{3}\right), 1.73$ $\left(\mathrm{s}, 6 \mathrm{H}, 2 \mathrm{CH}_{3}\right), 2.55\left(\mathrm{~s}, 3 \mathrm{H}, \mathrm{CH}_{3}\right), 2.60\left(\mathrm{~s}, 3 \mathrm{H}, \mathrm{CH}_{3}\right), 3.98\left(\mathrm{~s}, 3 \mathrm{H}, \mathrm{OCH}_{3}\right), 7.44\left(\mathrm{~d},{ }^{3} J_{\mathrm{H}, \mathrm{H}}=8.3 \mathrm{~Hz}, 1 \mathrm{H}\right.$, aromatic), $8.03\left(\mathrm{~s}, 1 \mathrm{H}\right.$, aromatic), $8.15\left(\mathrm{~d},{ }^{3} J_{\mathrm{H}, \mathrm{H}}=8.2 \mathrm{~Hz}, 1 \mathrm{H}\right.$, aromatic), $12.84(\mathrm{~s}, 1 \mathrm{H}, \mathrm{NH}), 12.97(\mathrm{~s}, 1 \mathrm{H}$, $\mathrm{NH}) ;{ }^{13} \mathrm{C}\left\{{ }^{1} \mathrm{H}\right\}$-NMR $\left(\mathrm{CDCl}_{3} ; 125.78 \mathrm{MHz}\right) \delta=19.7,19.9,26.7,26.8,53.0,88.0,88.4,103.5,103.6,127.5$, $129.2,130.2,130.8,132.3,136.4,162.3,162.4,165.0,167.7,167.7,172.6$; UV-Vis (methanol; $\lambda$ [nm] $(\log \varepsilon)$ ): 323 (4.61), 289 (4.96), 226 (4.75), 203 (4.65).

Ethyl 3,4-bis((1-(2,2-dimethyl-4,6-dioxo-1,3-dioxan-5-ylidene)ethyl)amino)benzoate (2o); white, $0.9 \mathrm{~g}$ $(1.7 \mathrm{mmol}, 31 \%) ; \mathrm{m} \cdot \mathrm{p}_{\mathrm{dec}} \cdot=171.4{ }^{\circ} \mathrm{C} ;{ }^{1} \mathrm{H}-\mathrm{NMR}\left(\mathrm{CDCl}_{3} ; 400.2 \mathrm{MHz}\right) \delta=1.42\left(\mathrm{t},{ }^{3} J_{\mathrm{H}, \mathrm{H}}=7.0 \mathrm{~Hz}\right.$, $\left.3 \mathrm{H}, \mathrm{CH}_{3}\right), 1.71\left(\mathrm{~s}, 6 \mathrm{H}, 2 \mathrm{CH}_{3}\right), 1.72\left(\mathrm{~s}, 6 \mathrm{H}, 2 \mathrm{CH}_{3}\right), 2.54\left(\mathrm{~s}, 3 \mathrm{H}, \mathrm{CH}_{3}\right), 2.59\left(\mathrm{~s}, 3 \mathrm{H}, \mathrm{CH}_{3}\right), 4.43\left(\mathrm{q},{ }^{3} J_{\mathrm{H}, \mathrm{H}}=7.1\right.$ $\left.\mathrm{Hz}, 2 \mathrm{H}, \mathrm{OCH}_{2}\right), 7.43\left(\mathrm{~d},{ }^{3} J_{\mathrm{H}, \mathrm{H}}=8.2 \mathrm{~Hz}, 1 \mathrm{H}\right.$, aromatic), $8.02\left(\mathrm{~s}, 1 \mathrm{H}\right.$, aromatic), $8.15\left(\mathrm{~d},{ }^{3} J_{\mathrm{H}, \mathrm{H}}=8.1 \mathrm{~Hz}\right.$, $1 \mathrm{H}$, aromatic), $12.82(\mathrm{~s}, 1 \mathrm{H}, \mathrm{NH}), 12.95(\mathrm{~s}, 1 \mathrm{H}, \mathrm{NH}) ;{ }^{13} \mathrm{C}\left\{{ }^{1} \mathrm{H}\right\}-\mathrm{NMR}\left(\mathrm{CDCl}_{3} ; 125.78 \mathrm{MHz}\right) \delta=14.4,19.7$, $19.9,26.7,26.7,62.1,87.9,88.3,103.5,103.5,127.5,129.2,130.2,131.2,132.2,136.3,162.3,162.4,164.4$, 167.7, 167.7, 172.6, 173.3; UV-Vis (methanol; $\lambda$ [nm] (loge)): 321 (4.25), 287 (4.63), 225 (4.44), 204 (4.35).

1H-benzo[d]imidazole-6-carboxylic acid; m.p dec. $=278.0-280.0{ }^{\circ} \mathrm{C} ;{ }^{1} \mathrm{H}-\mathrm{NMR}$ (DMSO-d6/KOD; $500.2 \mathrm{MHz}$ ) $\delta=7.74\left(\mathrm{~d},{ }^{3} J_{\mathrm{H}, \mathrm{H}}=8.7 \mathrm{~Hz}, 1 \mathrm{H}\right.$, aromatic), $7.96\left(\mathrm{~d},{ }^{3} J_{\mathrm{H}, \mathrm{H}}=8.7 \mathrm{~Hz},{ }^{4} J_{\mathrm{H}, \mathrm{H}}=1.4 \mathrm{~Hz}, 1 \mathrm{H}\right.$, aromatic), $8.23(\mathrm{~s}$, $1 \mathrm{H}$, aromatic), $9.08\left(\mathrm{~s}, 1 \mathrm{H}\right.$, aromatic); ${ }^{1} \mathrm{H}-\mathrm{NMR}\left(\mathrm{D}_{2} \mathrm{O} / \mathrm{KOD} ; 500.18 \mathrm{MHz}\right) \delta=7.66\left(\mathrm{~d},{ }^{3} \mathrm{~J}_{\mathrm{H}, \mathrm{H}}=8.5 \mathrm{~Hz}, 1 \mathrm{H}\right.$, aromatic), $7.81\left(\mathrm{dd},{ }^{3} J_{\mathrm{H}, \mathrm{H}}=8.5 \mathrm{~Hz},{ }^{4} J_{\mathrm{H}, \mathrm{H}}=1.5 \mathrm{~Hz}, 1 \mathrm{H}\right.$, aromatic), $8.21(\mathrm{~s}, 1 \mathrm{H}$, aromatic), $8.36(\mathrm{~s}, 1 \mathrm{H}$, aromatic); ${ }^{13} \mathrm{C}\left\{{ }^{1} \mathrm{H}\right\}$-NMR (DMSO-d6/KOD; $\left.125.78 \mathrm{MHz}\right) \delta=116.0,117.9,128.4,130.4,132.1,135.0,143.1$, $170.8 ;{ }^{13} \mathrm{C}\left\{{ }^{1} \mathrm{H}\right\}-\mathrm{NMR}\left(\mathrm{D}_{2} \mathrm{O} / \mathrm{KOD} ; 125.78 \mathrm{MHz}\right) \delta=115.3$ (bs), 118.3 (bs), 123.8, 124.8, 144.7, 168.4; HRMS (IT TOF): $\mathrm{m} / \mathrm{z}$ Calcd for $\mathrm{C}_{8} \mathrm{H}_{7} \mathrm{~N}_{2} \mathrm{O}_{2}[\mathrm{M}+\mathrm{H}]^{+}=163.0508$, Found 163.0509.

\subsubsection{Step B}

Modifications to existing procedures described in the literature [22,23] rely on purifications. The crude products were extracted with hexane or toluene at Soxhlet apparatus. Into freshly distillated diphenyl ether $(50 \mathrm{~mL})$ at $240{ }^{\circ} \mathrm{C}$ was added compound $2(10.0 \mathrm{mmol})$ in small portions, resulting in vigorous gas evolution. The resulting orange solution was brought to reflux for $30 \mathrm{~min}$, and was then allowed to cool to $70{ }^{\circ} \mathrm{C}$. The acetone or hexane $(25 \mathrm{~mL})$ was added and a dark-brown solid precipitated was filtered, washed with acetone $(2 \times 10 \mathrm{~mL})$. The crude product was extracted with hexane or toluene at Soxhlet apparatus to yield solid as follows:

1,10-Dihydro-1,10-phenanthroline-4,7-dione (3a); [41] brown; $1.4 \mathrm{~g}(6.8 \mathrm{mmol}, 68 \%)$; m.pdec. $>350{ }^{\circ} \mathrm{C} ;{ }^{1} \mathrm{H}$ $\operatorname{NMR}\left(\mathrm{CD}_{3} \mathrm{OD} / \mathrm{KOD} / \mathrm{D}_{2} \mathrm{O} ; 400.2 \mathrm{MHz}\right) \delta=6.62\left(\mathrm{~d},{ }^{3} J_{\mathrm{H}, \mathrm{H}}=5.6 \mathrm{~Hz}, 2 \mathrm{H}\right.$, aromatic), 8.08 (s, 2H, aromatic), $8.36\left(\mathrm{~d},{ }^{3} J_{\mathrm{H}, \mathrm{H}}=5.6 \mathrm{~Hz}, 2 \mathrm{H}\right.$, aromatic); ${ }^{13} \mathrm{C}\left\{{ }^{1} \mathrm{H}\right\}-\mathrm{NMR}\left(\mathrm{CD}_{3} \mathrm{OD} / \mathrm{KOD} / \mathrm{D}_{2} \mathrm{O} ; 100.5 \mathrm{MHz}\right) \delta=111.6,118.8$, $127.0,148.8,150.5,174.7$.

5-Fluoro-1,10-dihydro-1,10-phenanthroline-4,7-dione (3b); brown; $1.8 \mathrm{~g}(7.7 \mathrm{mmol}, 77 \%)$; $\mathrm{m} \cdot \mathrm{p}_{\mathrm{dec}} .>350.0^{\circ} \mathrm{C}$; ${ }^{1} \mathrm{H}-\mathrm{NMR}\left(\mathrm{CD}_{3} \mathrm{OD} / \mathrm{KOD} / \mathrm{D}_{2} \mathrm{O} ; 400.2 \mathrm{MHz}\right) \delta=6.45\left(\mathrm{~d},{ }^{3} \mathrm{~J}_{\mathrm{H}, \mathrm{H}}=6.5 \mathrm{~Hz}, 1 \mathrm{H}\right.$, aromatic), $6.50\left(\mathrm{~d},{ }^{3} J_{\mathrm{H}, \mathrm{H}}=6.2\right.$ $\mathrm{Hz}, 1 \mathrm{H}$, aromatic), $7.31\left(\mathrm{~d},{ }^{3} J_{\mathrm{H}, \mathrm{F}}=14.1 \mathrm{~Hz}, 1 \mathrm{H}\right.$, aromatic), $8.01\left(\mathrm{~d},{ }^{3} J_{\mathrm{H}, \mathrm{H}}=6.4 \mathrm{~Hz}, 1 \mathrm{H}\right.$, aromatic), $8.10(\mathrm{~d}$, 
${ }^{3} J_{\mathrm{H}, \mathrm{H}}=6.1 \mathrm{~Hz}, 1 \mathrm{H}$, aromatic); ${ }^{13} \mathrm{C}\left\{{ }^{1} \mathrm{H}\right\}-\mathrm{NMR}\left(\mathrm{CD}_{3} \mathrm{OD} / \mathrm{KOD} / \mathrm{D}_{2} \mathrm{O} ; 100.5 \mathrm{MHz}\right) \delta=101.1\left(\mathrm{~d},{ }^{2} J_{\mathrm{C}, \mathrm{F}}=24.1\right.$ $\mathrm{Hz}), 113.0\left(\mathrm{~d},{ }^{1} J_{\mathrm{C}, \mathrm{F}}=307.7 \mathrm{~Hz}\right), 117.6\left(\mathrm{~d},{ }^{2} J_{\mathrm{C}, \mathrm{F}}=11.3 \mathrm{~Hz}\right), 125.0\left(\mathrm{~d},{ }^{3} J_{\mathrm{C}, \mathrm{F}}=9.6 \mathrm{~Hz}\right), 130.7,136.4,142.9$, 143.4, 147.9, 156.4, 158.9, 175.7, 176.9; GC-MS: $\mathrm{t}_{\mathrm{r}}=5.1 \mathrm{~min},(\mathrm{EI}) \mathrm{M}^{+}=230(<1 \%) ;{ }^{19} \mathrm{~F}\left\{{ }^{1} \mathrm{H}\right\}-\mathrm{NMR}$ $\left(\mathrm{CD}_{3} \mathrm{OD} / \mathrm{KOD} / \mathrm{D}_{2} \mathrm{O} ; 470.5 \mathrm{MHz}\right) \delta=-119.68 ;{ }^{19} \mathrm{~F}-\mathrm{NMR}\left(\mathrm{CD}_{3} \mathrm{OD} / \mathrm{KOD} / \mathrm{D}_{2} \mathrm{O} ; 470.5 \mathrm{MHz}\right) \delta=-119.68$ $\left(\mathrm{d},{ }^{3} J_{\mathrm{F}, \mathrm{H}}=13.8 \mathrm{~Hz}\right) ; \mathrm{UV}-\mathrm{Vis}$ (methanol; $\lambda$ [nm] (loge)): 352 (3.40), 329 (3.61), 308 (3.55), 273 (3.56), 251 (3.63), 224 (3.91), 208 (3.96).

5-Chloro-1,10-dihydro-1,10-phenanthroline-4,7-dione (3c); brown; [23]; $1.5 \mathrm{~g}$ (6.1 mmol, 61\%); m.p dec $=272.8-273.6{ }^{\circ} \mathrm{C} ;{ }^{1} \mathrm{H}-\mathrm{NMR}\left(\mathrm{CD}_{3} \mathrm{OD} / \mathrm{KOD} / \mathrm{D}_{2} \mathrm{O} ; 400.2 \mathrm{MHz}\right) \delta=6.62\left(\mathrm{~d},{ }^{3} J_{\mathrm{H}, \mathrm{H}}=6.9 \mathrm{~Hz}\right.$, $1 \mathrm{H}$, aromatic), $6.64\left(\mathrm{~d},{ }^{3} J_{\mathrm{H}, \mathrm{H}}=6.4 \mathrm{~Hz}, 1 \mathrm{H}\right.$, aromatic), $8.27\left(\mathrm{~d},{ }^{3} J_{\mathrm{H}, \mathrm{H}}=5.9 \mathrm{~Hz}, 1 \mathrm{H}\right.$, aromatic), 8.31 (d, ${ }^{3} J_{\mathrm{H}, \mathrm{H}}=5.8 \mathrm{~Hz}, 1 \mathrm{H}$, aromatic $) ;{ }^{13} \mathrm{C}\left\{{ }^{1} \mathrm{H}\right\}-\mathrm{NMR}\left(\mathrm{CD}_{3} \mathrm{OD} / \mathrm{KOD} / \mathrm{D}_{2} \mathrm{O} ; 100.5 \mathrm{MHz}\right) \delta=112.0,114.5,120.3$, 122.5, 125.4, 126.1, 144.0, 147.1, 148.6, 149.4, 169.3, 173.7, 173.8, 176.0, 176.1; GC-MS: $\mathrm{t}_{\mathrm{r}}=11.5 \mathrm{~min},(\mathrm{EI})$ $\mathrm{M}^{+}=246(100 \%), 248(32 \%),[\mathrm{M}+\mathrm{H}]^{+}=247(8 \%),[\mathrm{M}-\mathrm{CO}]^{+}=218(90 \%) ; \mathrm{UV}-\mathrm{Vis}$ (methanol; $\lambda[\mathrm{nm}]$ $(\log \varepsilon)): 356$ (3.83), 338 (4.06), 324 (4.06), 285 (3.97), 273 (4.03), 249 (4.42), 231 (4.28), 215 (4.42).

5-Methyl-1,10-dihydro-1,10-phenanthroline-4,7-dione (3d); brown; $1.9 \mathrm{~g}(8.6 \mathrm{mmol}, 86 \%) ; \mathrm{m} . \mathrm{p}_{\mathrm{dec}} .>350.0^{\circ} \mathrm{C}$; ${ }^{1} \mathrm{H}-\mathrm{NMR}\left(\mathrm{CD}_{3} \mathrm{OD} / \mathrm{KOD} / \mathrm{D}_{2} \mathrm{O} ; 500.2 \mathrm{MHz}\right) \delta=2.73\left(\mathrm{~d},{ }^{4} J_{\mathrm{H}, \mathrm{H}}=0.8 \mathrm{~Hz}, 3 \mathrm{H}, \mathrm{CH}_{3}\right), 6.36\left(\mathrm{~d},{ }^{3} J_{\mathrm{H}, \mathrm{H}}=6.6 \mathrm{~Hz}\right.$, $1 \mathrm{H}$, aromatic), $6.48\left(\mathrm{~d},{ }^{3} J_{\mathrm{H}, \mathrm{H}}=6.0 \mathrm{~Hz}, 1 \mathrm{H}\right.$, aromatic), $7.47\left(\mathrm{~d},{ }^{4} J_{\mathrm{H}, \mathrm{H}}=1.0 \mathrm{~Hz}, 1 \mathrm{H}\right.$, aromatic), $7.88(\mathrm{~d}$, ${ }^{3} J_{\mathrm{H}, \mathrm{H}}=6.6 \mathrm{~Hz}, 1 \mathrm{H}$, aromatic), $8.08\left(\mathrm{~d},{ }^{3} J_{\mathrm{H}, \mathrm{H}}=6.0 \mathrm{~Hz}, 1 \mathrm{H}\right.$, aromatic); ${ }^{13} \mathrm{C}\left\{{ }^{1} \mathrm{H}\right\}-\mathrm{NMR}\left(\mathrm{CD}_{3} \mathrm{OD} / \mathrm{KOD} / \mathrm{D}_{2} \mathrm{O}\right.$; $125.78 \mathrm{MHz}) \delta=24.5,112.0,114.0,119.9,124.9,125.9,132.6,139.8,141.0,142.5,148.0,174.6,180.9$; GC-MS: $\mathrm{t}_{\mathrm{r}}=7.0 \mathrm{~min},(\mathrm{EI})[\mathrm{M}-\mathrm{H}]^{+}=225(1 \%)$; UV-Vis (methanol; $\left.\lambda[\mathrm{nm}](\log \varepsilon)\right): 355(3.66), 327$ (3.89), 306 (3.83), 275 (3.93), 247 (4.14), 224 (4.23), 209 (4.28).

4,7-Dioxo-1,4,7,10-tetrahydro-1,10-phenanthroline-5-carbonitrile (3e); beige, $1.5 \mathrm{~g}(6.4 \mathrm{mmol}, 64 \%)$; $\mathrm{m} . \mathrm{p}_{\text {dec }}$. $>350{ }^{\circ} \mathrm{C} ;{ }^{1} \mathrm{H}-\mathrm{NMR}\left(\mathrm{DMSO}-d_{6} / \mathrm{KOD} / \mathrm{D}_{2} \mathrm{O} ; 400.2 \mathrm{MHz}\right) \delta=6.37\left(\mathrm{~d},{ }^{3} \mathrm{~J}_{\mathrm{H}, \mathrm{H}}=6.8 \mathrm{~Hz}, 1 \mathrm{H}\right.$, aromatic), 6.41 $\left(\mathrm{d},{ }^{3} J_{\mathrm{H}, \mathrm{H}}=6.3 \mathrm{~Hz}, 1 \mathrm{H}\right.$, aromatic), $8.02\left(\mathrm{~d},{ }^{3} J_{\mathrm{H}, \mathrm{H}}=6.7 \mathrm{~Hz}, 1 \mathrm{H}\right.$, aromatic), $8.16\left(\mathrm{~d},{ }^{3} J_{\mathrm{H}, \mathrm{H}}=6.3 \mathrm{~Hz}, 1 \mathrm{H}\right.$, aromatic), $8.26\left(\mathrm{~s}, 1 \mathrm{H}\right.$, aromatic); ${ }^{13} \mathrm{C}\left\{{ }^{1} \mathrm{H}\right\}-\mathrm{NMR}\left(\mathrm{DMSO}-d_{6} / \mathrm{KOD} / \mathrm{D}_{2} \mathrm{O} ; 125.78 \mathrm{MHz}\right) \delta=101.1,113.7$, $114.2,122.2,123.3,124.8,131.2,139.6,142.2,144.6,149.7,176.1,176.8$; UV-Vis (methanol; $\lambda$ [nm] $(\log \varepsilon)$ ): 382 (3.27), 350 (3.50), 335 (3.44), 277 (3.39), 242 (3.78), 214 (3.81).

5-Fluoro-2,9-dimethyl-1,5,10,10a-tetrahydro-1,10-phenanthroline-4,7-dione (3f); brown; $2.2 \mathrm{~g}$ (8.7 mmol, $87 \%) ; \mathrm{m} . \mathrm{p}_{\text {dec }}=370.2-372.8^{\circ} \mathrm{C} ;{ }^{1} \mathrm{H}-\mathrm{NMR}\left(\mathrm{CD}_{3} \mathrm{OD} / \mathrm{KOD} / \mathrm{D}_{2} \mathrm{O} ; 400.2 \mathrm{MHz}\right) \delta=2.40\left(\mathrm{bs}, 6 \mathrm{H}, \mathrm{CH}_{3}\right), 6.26$ (bs, $1 \mathrm{H}$, aromatic), 6.35 (bs, $1 \mathrm{H}$, aromatic), $7.19\left(\mathrm{bd},{ }^{3} J_{\mathrm{H}, \mathrm{F}}=11.1 \mathrm{~Hz}, 1 \mathrm{H}\right.$, aromatic); ${ }^{13} \mathrm{C}\left\{{ }^{1} \mathrm{H}\right\}-\mathrm{NMR}$ $\left(\mathrm{CD}_{3} \mathrm{OD} / \mathrm{KOD} / \mathrm{D}_{2} \mathrm{O} ; 100.5 \mathrm{MHz}\right) \delta=21.4,22.9,100.4\left(\mathrm{~d},{ }^{2} J_{\mathrm{C}, \mathrm{F}}=24.2 \mathrm{~Hz}\right), 112.2\left(\mathrm{~d},{ }^{1} J_{\mathrm{C}, \mathrm{F}}=274.4 \mathrm{~Hz}\right)$, $115.5\left(\mathrm{~d},{ }^{2} J_{\mathrm{C}, \mathrm{F}}=11.1 \mathrm{~Hz}\right), 123.1\left(\mathrm{~d},{ }^{3} J_{\mathrm{C}, \mathrm{F}}=9.7 \mathrm{~Hz}\right), 135.0,141.5\left(\mathrm{~d},{ }^{4} J_{\mathrm{C}, \mathrm{F}}=3.7 \mathrm{~Hz}\right), 154.0,155.8,157.8$, 158.3, 175.0, 176.2, 176.2; GC-MS: $\mathrm{t}_{\mathrm{r}}=10.5 \mathrm{~min},(\mathrm{EI}) \mathrm{M}^{+}=258(100 \%),[\mathrm{M}+\mathrm{H}-\mathrm{CO}]^{+}=229(98 \%)$.

2,9-Dimethyl-1,10-phenanthroline-4,7(1H,10H)-dione (3g); brown; [22]; $1.6 \mathrm{~g}$ (6.8 mmol, 68\%); m.p.ec $=291.8-292.5{ }^{\circ} \mathrm{C} ;{ }^{1} \mathrm{H}-\mathrm{NMR}\left(\mathrm{CD}_{3} \mathrm{OD} / \mathrm{KOD} / \mathrm{D}_{2} \mathrm{O} ; 400.2 \mathrm{MHz}\right) \delta=2.29\left(\mathrm{~s}, 6 \mathrm{H}, \mathrm{CH}_{3}\right), 6.24$ (s, $2 \mathrm{H}$, aromatic), $7.64\left(\mathrm{~s}, 2 \mathrm{H}\right.$, aromatic); ${ }^{13} \mathrm{C}\left\{{ }^{1} \mathrm{H}\right\}-\mathrm{NMR}\left(\mathrm{CD}_{3} \mathrm{OD} / \mathrm{KOD} / \mathrm{D}_{2} \mathrm{O} ; 100.5 \mathrm{MHz}\right) \delta=21.8,100.0$, $110.1,117.0,122.9,138.9,155.9,174.6$.

5-Fluoro-2,9-dimethyl-1,5,10,10a-tetrahydro-1,10-phenanthroline-4,7-dione (3h); brown; $2.2 \mathrm{~g}$ (8.7 mmol, $87 \%) ; \mathrm{m} \cdot \mathrm{p}_{\text {dec }}=370.2-372.8^{\circ} \mathrm{C} ;{ }^{1} \mathrm{H}-\mathrm{NMR}\left(\mathrm{CD}_{3} \mathrm{OD} / \mathrm{KOD} / \mathrm{D}_{2} \mathrm{O} ; 400.2 \mathrm{MHz}\right) \delta=2.40\left(\mathrm{bs}, 6 \mathrm{H}, \mathrm{CH}_{3}\right), 6.26$ (bs, $1 \mathrm{H}$, aromatic), 6.35 (bs, $1 \mathrm{H}$, aromatic), $7.19\left(\mathrm{bd},{ }^{3} \mathrm{~J}_{\mathrm{H}, \mathrm{F}}=11.1 \mathrm{~Hz}, 1 \mathrm{H}\right.$, aromatic); ${ }^{13} \mathrm{C}\left\{{ }^{1} \mathrm{H}\right\}-\mathrm{NMR}$ $\left(\mathrm{CD}_{3} \mathrm{OD} / \mathrm{KOD} / \mathrm{D}_{2} \mathrm{O} ; 100.5 \mathrm{MHz}\right) \delta=21.4,22.9,100.4\left(\mathrm{~d},{ }^{2} J_{\mathrm{C}, \mathrm{F}}=24.2 \mathrm{~Hz}\right), 112.2\left(\mathrm{~d},{ }^{1} J_{\mathrm{C}, \mathrm{F}}=274.4 \mathrm{~Hz}\right)$, $115.5\left(\mathrm{~d},{ }^{2} J_{\mathrm{C}, \mathrm{F}}=11.1 \mathrm{~Hz}\right), 123.1\left(\mathrm{~d},{ }^{3} J_{\mathrm{C}, \mathrm{F}}=9.7 \mathrm{~Hz}\right), 135.0,141.5\left(\mathrm{~d},{ }^{4} J_{\mathrm{C}, \mathrm{F}}=3.7 \mathrm{~Hz}\right), 154.0,155.8,157.8$, 158.3, 175.0, 176.2, 176.2; GC-MS: $\mathrm{t}_{\mathrm{r}}=10.5 \mathrm{~min},(\mathrm{EI}) \mathrm{M}^{+}=258(100 \%),[\mathrm{M}+\mathrm{H}-\mathrm{CO}]^{+}=229(98 \%)$; ${ }^{19} \mathrm{~F}\left\{{ }^{1} \mathrm{H}\right\}-\mathrm{NMR}\left(\mathrm{CD}_{3} \mathrm{OD} / \mathrm{KOD} / \mathrm{D}_{2} \mathrm{O} ; 470.5 \mathrm{MHz}\right) \delta=-119.99 ;{ }^{19} \mathrm{~F}-\mathrm{NMR}\left(\mathrm{CD}_{3} \mathrm{OD} / \mathrm{KOD} / \mathrm{D}_{2} \mathrm{O} ; 470.5 \mathrm{MHz}\right)$ $\delta=-119.99\left(\mathrm{~d},{ }^{3} J_{\mathrm{F}, \mathrm{H}}=13.9 \mathrm{~Hz}\right)$; UV-Vis (methanol; $\left.\lambda[\mathrm{nm}](\log \varepsilon)\right): 353$ (3.76), 337 (3.85), 319 (4.00), 311 (3.97), 270 (4.21), 254 (4.46), 225 (4.31), 214 (4.44).

5-Chloro-2,9-dimethyl-1,5,10,10a-tetrahydro-1,10-phenanthroline-4,7-dione (3i); $2.3 \mathrm{~g}$ (8.3 mmol, 83\%); brown; m.p $\mathrm{pdec}=332.7-334.8^{\circ} \mathrm{C} ;{ }^{1} \mathrm{H}-\mathrm{NMR}\left(\mathrm{CD}_{3} \mathrm{OD} / \mathrm{KOD} / \mathrm{D}_{2} \mathrm{O} ; 400.2 \mathrm{MHz}\right) \delta=2.46\left(\mathrm{~s}, 3 \mathrm{H}, \mathrm{CH}_{3}\right)$, 
$2.49\left(\mathrm{~s}, 3 \mathrm{H}, \mathrm{CH}_{3}\right), 6.40\left(\mathrm{~s}, 1 \mathrm{H}\right.$, aromatic), $6.41\left(\mathrm{~s}, 1 \mathrm{H}\right.$, aromatic), $7.78\left(\mathrm{~s}, 1 \mathrm{H}\right.$, aromatic); ${ }^{13} \mathrm{C}\left\{{ }^{1} \mathrm{H}\right\}-\mathrm{NMR}$ $\left(\mathrm{CD}_{3} \mathrm{OD} / \mathrm{KOD} / \mathrm{D}_{2} \mathrm{O} ; 100.5 \mathrm{MHz}\right) \delta=21.8,22.6,112.0,114.3,120.1,121.1,124.2,126.2,138.4,141.6$, 155.2, 156.8, 175.9, 178.1; GC-MS: $\mathrm{t}_{\mathrm{r}}=11.3 \mathrm{~min},(\mathrm{EI}) \mathrm{M}^{+}=273.9(100 \%),[\mathrm{M}-\mathrm{CO}]^{+}=245(80 \%)$; UV-Vis (methanol; $\lambda$ [nm] (loge)): 357 (3.65), 340 (3.84), 327 (3.96), 316 (3.94), 280 (4.00), 254 (4.35), 230 (4.25), 216 (4.35).

5-Bromo-2,9-dimethyl-1,5,10,10a-tetrahydro-1,10-phenanthroline-4,7-dione (3j); brown; $2.7 \mathrm{~g}$ (8.5 mmol, $85 \%) ; \mathrm{m} . \mathrm{p}_{\mathrm{dec}} \cdot=325.9-326.6{ }^{\circ} \mathrm{C} ;{ }^{1} \mathrm{H}-\mathrm{NMR}\left(\mathrm{CD}_{3} \mathrm{OD} / \mathrm{KOD} / \mathrm{D}_{2} \mathrm{O} ; 400.2 \mathrm{MHz}\right) \delta=2.48\left(\mathrm{~s}, 3 \mathrm{H}, \mathrm{CH}_{3}\right)$, $2.50\left(\mathrm{~s}, 3 \mathrm{H}, \mathrm{CH}_{3}\right), 6.40$ (s, $1 \mathrm{H}$, aromatic), $6.43\left(\mathrm{~s}, 1 \mathrm{H}\right.$, aromatic), $8.09\left(\mathrm{~s}, 1 \mathrm{H}\right.$, aromatic); ${ }^{13} \mathrm{C}\left\{{ }^{1} \mathrm{H}\right\}-\mathrm{NMR}$ $\left(\mathrm{CD}_{3} \mathrm{OD} / \mathrm{KOD} / \mathrm{D}_{2} \mathrm{O} ; 100.5 \mathrm{MHz}\right) \delta=21.6,22.7,112.2,112.7,113.9,121.4,124.4,124.9,138.9,141.1,154.4$, 157.1, 175.8, 175.8, 178.2; GC-MS: $\mathrm{t}_{\mathrm{r}}=13.5 \mathrm{~min},(\mathrm{EI}) \mathrm{M}^{+}=318(100 \%), 320(96 \%),[\mathrm{M}-\mathrm{CO}]^{+}=290(63 \%)$; UV-Vis (methanol; $\lambda$ [nm] (loge)): 356 (3.87), 339 (4.08), 328 (4.17), 315 (4.13), 281 (4.19), 254 (4.55), 231 (4.46), 217 (4.53).

2,5,9-Trimethyl-1,10-dihydro-1,10-phenanthroline-4,7-dione (3k); brown; $2.3 \mathrm{~g}$ (9.2 mmol, 92\%); m.p $\mathrm{pec}_{\text {dec }}=337.4-338.9{ }^{\circ} \mathrm{C} ;{ }^{1} \mathrm{H}-\mathrm{NMR}\left(\mathrm{CD}_{3} \mathrm{OD} / \mathrm{KOD} / \mathrm{D}_{2} \mathrm{O} ; 400.2 \mathrm{MHz}\right) \delta=2.31\left(\mathrm{~s}, 3 \mathrm{H}, \mathrm{CH}_{3}\right), 2.36$ $\left(\mathrm{s}, 3 \mathrm{H}, \mathrm{CH}_{3}\right), 2.66\left(\mathrm{~s}, 3 \mathrm{H}, \mathrm{OCH}_{3}\right), 6.16(\mathrm{~s}, 1 \mathrm{H}$, aromatic), 6.31 (s, 1H, aromatic), 7.28 (s, $1 \mathrm{H}$, aromatic); ${ }^{13} \mathrm{C}\left\{{ }^{1} \mathrm{H}\right\}$-NMR $\left(\mathrm{CD}_{3} \mathrm{OD} / \mathrm{KOD} / \mathrm{D}_{2} \mathrm{O} ; 100.5 \mathrm{MHz}\right) \delta=21.1,23.5,24.4,111.4,113.2,119.3,123.0,123.9,131.6$, 138.2, 139.6, 152.8, 157.5, 174.2, 180.3; GC-MS: $\mathrm{t}_{\mathrm{r}}=12.1 \mathrm{~min},(\mathrm{EI}) \mathrm{M}^{+}=254(100 \%),[\mathrm{M}+\mathrm{H}-\mathrm{CO}]^{+}=225$ (56\%); UV-Vis (methanol; $\lambda$ [nm] (loge)): 354 (3.72), 338 (3.95), 320 (4.14), 282 (4.30), 257 (4.45), 226 (4.40), 217 (4.42), 204 (4.40).

2,9-Dimethyl-4,7-dioxo-1,4,7,10-tetrahydro-1,10-phenanthroline-5-carbonitrile (31); brown; $1.6 \mathrm{~g}$ (6.2 mmol, $62 \%) ; \mathrm{m} . \mathrm{p}_{\text {dec }}=390.7-392.5{ }^{\circ} \mathrm{C} ;{ }^{1} \mathrm{H}-\mathrm{NMR}\left(\mathrm{CD}_{3} \mathrm{OD} / \mathrm{KOD} / \mathrm{D}_{2} \mathrm{O} ; 400.2 \mathrm{MHz}\right) \delta=2.45\left(\mathrm{~s}, 3 \mathrm{H}, \mathrm{CH}_{3}\right)$, $2.46\left(\mathrm{~s}, 3 \mathrm{H}, \mathrm{CH}_{3}\right), 6.45$ (s, $1 \mathrm{H}$, aromatic), $6.47\left(\mathrm{~s}, 1 \mathrm{H}\right.$, aromatic), $8.21\left(\mathrm{~s}, 1 \mathrm{H}\right.$, aromatic); ${ }^{13} \mathrm{C}\left\{{ }^{1} \mathrm{H}\right\}-\mathrm{NMR}$ $\left(\mathrm{CD}_{3} \mathrm{OD} / \mathrm{KOD} / \mathrm{D}_{2} \mathrm{O} ; 100.5 \mathrm{MHz}\right) \delta=23.8,100.0,112.1,112.2,122.0,122.9,123.3,130.5,144.5,146.7,159.1$, 159.2, 162.1, 169.4, 173.8, 174.1; GC-MS: $\mathrm{t}_{\mathrm{r}}=9.4 \mathrm{~min},(\mathrm{EI}) \mathrm{M}^{+}=265$ (5\%); UV-Vis (methanol; $\lambda$ [nm] $(\log \varepsilon)): 350(3.90), 343$ (3.93), 328 (3.87), 286 (3.82), 276 (3.90), 252 (4.31), 228 (4.15), 217 (4.28).

Methyl 2,9-dimethyl-4,7-dioxo-1,4,7,10-tetrahydro-1,10-phenanthroline-5-carboxylate (3m); brown; $2.3 \mathrm{~g}$ $(7.8 \mathrm{mmol}, 78 \%) ; \mathrm{m} . \mathrm{p}_{\mathrm{dec}}=293.8-294.3{ }^{\circ} \mathrm{C} ;{ }^{1} \mathrm{H}-\mathrm{NMR}\left(\mathrm{CD}_{3} \mathrm{OD} / \mathrm{KOD} / \mathrm{D}_{2} \mathrm{O} ; 400.2 \mathrm{MHz}\right) \delta=2.44(\mathrm{~s}, 6 \mathrm{H}$, $\left.2 \mathrm{CH}_{3}\right), 3.85\left(\mathrm{~s}, 3 \mathrm{H}, \mathrm{OCH}_{3}\right), 6.32(\mathrm{~s}, 1 \mathrm{H}$, aromatic), 6.44 (s, $1 \mathrm{H}$, aromatic), 7.78 (s, $1 \mathrm{H}$, aromatic); GC-MS: $\mathrm{t}_{\mathrm{r}}=9.3 \mathrm{~min},(\mathrm{EI})[\mathrm{M}-\mathrm{MeOH}]^{+}=266(100 \%)$; UV-Vis (methanol; $\left.\lambda[\mathrm{nm}](\log \varepsilon)\right): 345(3.83), 330(4.04), 314$ (3.98), 280 (4.03), 252 (4.43), 215 (4.36).

Ethyl 2,9-dimethyl-4,7-dioxo-1,4,7,10-tetrahydro-1,10-phenanthroline-5-carboxylate (3n); brown; $2.0 \mathrm{~g}$ $(6.5 \mathrm{mmol}, 65 \%) ; \mathrm{m} . \mathrm{p}_{\mathrm{dec}} \cdot=301.4-301.8^{\circ} \mathrm{C} ;{ }^{1} \mathrm{H}-\mathrm{NMR}\left(\mathrm{CD}_{3} \mathrm{OD} / \mathrm{KOD} / \mathrm{D}_{2} \mathrm{O} ; 400.2 \mathrm{MHz}\right) \delta=1.41(\mathrm{t}$, $\left.{ }^{3} J_{\mathrm{H}, \mathrm{H}}=7.1 \mathrm{~Hz}, 3 \mathrm{H}, \mathrm{OCH}_{2} \mathrm{CH}_{3}\right), 2.36\left(\mathrm{~s}, 3 \mathrm{H}, \mathrm{CH}_{3}\right), 2.38\left(\mathrm{~s}, 3 \mathrm{H}, \mathrm{CH}_{3}\right), 4.46\left(\mathrm{q},{ }^{3} \mathrm{~J}_{\mathrm{H}, \mathrm{H}}=7.0 \mathrm{~Hz}, 2 \mathrm{H}, \mathrm{OCH}_{2}\right)$, $6.29\left(\mathrm{~s}, 1 \mathrm{H}\right.$, aromatic), $6.37\left(\mathrm{~s}, 1 \mathrm{H}\right.$, aromatic), $7.79\left(\mathrm{~s}, 1 \mathrm{H}\right.$, aromatic); GC-MS: $\mathrm{t}_{\mathrm{r}}=17.9 \mathrm{~min},(\mathrm{EI}) \mathrm{M}^{+}=312$ (50\%); UV-Vis (methanol; $\lambda[\mathrm{nm}](\log \varepsilon)): 354$ (3.58), 345 (3.81), 330 (4.04), 316 (3.99), 279 (4.03), 252 (4.44), 227 (4.26), 214 (4.36).

\subsubsection{Step C}

Modifications to existing procedure described in the literature [22,23] rely on the evaporation of excess phosphoryl chloride under reduced pressure, and the addition of $\mathrm{CH}_{2} \mathrm{Cl}_{2}$ or $\mathrm{CHCl}_{3}$ into reaction mixture after alkalified by $\mathrm{NaOH}$ solution, because of the very exothermic hydrolysis of residual phosphoryl chloride. Next the crude products were purified by chromatography and crystallization from $\mathrm{CH}_{2} \mathrm{Cl}_{2}$.

Freshly distillated phosphoryl chloride $(82.0 \mathrm{~g}, 50 \mathrm{~mL}, 534.8 \mathrm{mmol})$ was mixed under argon with compounds $3(5.0 \mathrm{mmol})$ and the resulting solutions were stirred at $90{ }^{\circ} \mathrm{C}$ for $4 \mathrm{~h}$. The excess of phosphoryl chloride was slowly evaporated, under reduced pressure. The reaction mixture was slowly added to a well stirred mixture of ice $(50 \mathrm{~g})$ in water $(100 \mathrm{~mL})$. After stirring for $15 \mathrm{~min}$. the resulting reaction mixture was carefully brought to $\mathrm{pH} 13-14$ ( $\mathrm{pH} 7$ in the case of molecule $4 \mathrm{~g}$ ) by adding $\mathrm{NaOH}$ 
solution (40\%). The aqueous layer was extracted with $\mathrm{CH}_{2} \mathrm{Cl}_{2}(4 \times 10 \mathrm{~mL})$. The combined organic layers was separated and dried over $\mathrm{MgSO}_{4}$. Evaporation of the brown colored solvent afforded products 4 as light tan crystals. Next, the crude products were purified by chromatography on silica gel using methanol/dichloromethane as eluent and finally crystallization from $\mathrm{CH}_{2} \mathrm{Cl}_{2}$ to yield precipitates as follows:

4,7-Dichloro-1,10-phenanthroline (4a) [42,43]; beige; $1.0 \mathrm{~g}(4.2 \mathrm{mmol}, 84 \%) ; \mathrm{m} . \mathrm{p}_{\mathrm{dec}} .=254.8-255.0{ }^{\circ} \mathrm{C}$; UV-Vis (methanol; $\lambda$ [nm] $(\log \varepsilon)$ ): 300 (3.83), 264 (4.40), 239 (4.33), 225 (4.29), 202 (4.36); IR (KBr): $\tilde{v}=3422,3034,1563,1487,1418,835,720 \mathrm{~cm}^{-1}$ (Supporting Information Figure S1).

4,7-Dichloro-5-fluoro-1,10-phenanthroline (4b); beige; $1.1 \mathrm{~g}(4.2 \mathrm{mmol}, 85 \%) ; \mathrm{m} . \mathrm{p}_{\mathrm{dec}} .=217.8-218.0^{\circ} \mathrm{C}$; ${ }^{1} \mathrm{H}-\mathrm{NMR}\left(\mathrm{CDCl}_{3} ; 600.2 \mathrm{MHz}\right) \delta=7.75\left(\mathrm{~d},{ }^{3} \mathrm{~J}_{\mathrm{H}, \mathrm{H}}=4.7 \mathrm{~Hz}, 1 \mathrm{H}\right.$, aromatic $), 7.77\left(\mathrm{~d},{ }^{3} J_{\mathrm{H}, \mathrm{H}}=4.8 \mathrm{~Hz}, 1 \mathrm{H}\right.$, aromatic), $7.94\left(\mathrm{~d},{ }^{3} J_{\mathrm{H}, \mathrm{F}}=13.3 \mathrm{~Hz}, 1 \mathrm{H}\right.$, aromatic), $9.02\left(\mathrm{dd},{ }^{3} J_{\mathrm{H}, \mathrm{H}}=4.8 \mathrm{~Hz},{ }^{5} J_{\mathrm{H}, \mathrm{F}}=0.5 \mathrm{~Hz}, 1 \mathrm{H}\right.$, aromatic), $9.08\left(\mathrm{~d},{ }^{3} J_{\mathrm{H}, \mathrm{H}}=4.8 \mathrm{~Hz}, 1 \mathrm{H}\right.$, aromatic); ${ }^{13} \mathrm{C}\left\{{ }^{1} \mathrm{H}\right\}-\mathrm{NMR}\left(\mathrm{CDCl}_{3} ; 150.0 \mathrm{MHz}\right) \delta=106.7\left(\mathrm{~d},{ }^{2} J_{\mathrm{C}, \mathrm{F}}=25.9 \mathrm{~Hz}\right)$, $119.9\left(\mathrm{~d},{ }^{2} J_{\mathrm{C}, \mathrm{F}}=12.6 \mathrm{~Hz}\right), 124.4,126.3,126.8\left(\mathrm{~d},{ }^{3} J_{\mathrm{C}, \mathrm{F}}=10.8 \mathrm{~Hz}\right), 140.3,142.0\left(\mathrm{~d},{ }^{3} J_{\mathrm{C}, \mathrm{F}}=6.2 \mathrm{~Hz}\right), 144.7$, $148.9\left(\mathrm{~d},{ }^{4} J_{\mathrm{C}, \mathrm{F}}=2.3 \mathrm{~Hz}\right), 149.7\left(\mathrm{~d},{ }^{4} J_{\mathrm{C}, \mathrm{F}}=2.6 \mathrm{~Hz}\right), 151.0,156.3\left(\mathrm{~d},{ }^{1} J_{\mathrm{C}, \mathrm{F}}=263.6 \mathrm{~Hz}\right) ;{ }^{19} \mathrm{~F}\left\{{ }^{1} \mathrm{H}\right\}-\mathrm{NMR}\left(\mathrm{CDCl}_{3} ;\right.$ $470.5 \mathrm{MHz}) \delta=-108.98 ;{ }^{19} \mathrm{~F}-\mathrm{NMR}\left(\mathrm{CDCl}_{3} ; 470.5 \mathrm{MHz}\right) \delta=-108.98\left(\mathrm{~d},{ }^{3} \mathrm{~J}_{\mathrm{F}, \mathrm{H}}=13.4 \mathrm{~Hz}\right) ; \mathrm{GC}-\mathrm{MS}: \mathrm{t}_{\mathrm{r}}=9.0$ min, (EI) $\mathrm{M}^{+}=266.0$ (100\%); Anal. Calcd for $\mathrm{C}_{12} \mathrm{H}_{5} \mathrm{~N}_{2} \mathrm{Cl}{ }_{2} \mathrm{~F}: \mathrm{C}, 53.96 ; \mathrm{H}, 1.89 ; \mathrm{N}, 10.49$; Found: $\mathrm{C}, 53.90$; $\mathrm{H}, 1.93$; N, 10.45; UV-Vis (methanol; $\lambda$ [nm] (loge)): 305 (4.01), 268 (4.46), 240 (4.47), 225 (4.40), 203 (4.45); IR (KBr): $\tilde{v}=3422,3035,1626,1571,1546,1410,842,752 \mathrm{~cm}^{-1}$ (Supporting Information Figure S2).

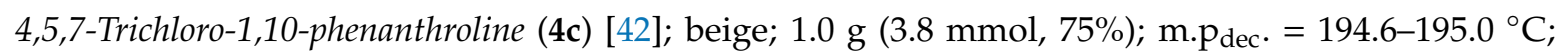
GC-MS: $\mathrm{t}_{\mathrm{r}}=9.4 \mathrm{~min},(\mathrm{EI}) \mathrm{M}^{+}=281.9(100 \%), 283.9(95 \%),[\mathrm{M}-\mathrm{Cl}]^{+}=247(46 \%) ; \mathrm{UV}-\mathrm{Vis}$ (methanol; $\lambda$ [nm] (loge)): 314 (3.82), 302 (3.87), 270 (4.32), 247 (4.31), 209 (4.30); IR (KBr): $\tilde{v}=3356,3021,2920,2191$, $1567,1403,833,716 \mathrm{~cm}^{-1}$ (Supporting Information Figure S3).

4,7-Dichloro-5-methyl-1,10-phenanthroline (4d) [23]; beige; $1.2 \mathrm{~g}(4.6 \mathrm{mmol}, 91 \%) ; \mathrm{m} \cdot \mathrm{p}_{\mathrm{dec}}=166.8-167.0^{\circ} \mathrm{C}$; GC-MS: $t_{\mathrm{r}}=9.6 \mathrm{~min},(\mathrm{EI}) \mathrm{M}^{+}=262.1(100 \%)$; UV-Vis (methanol; $\lambda$ [nm] $(\log \varepsilon)$ ): 304 (4.09), 271 (4.50), 241 (4.49), 225 (4.42), 205 (4.47); IR (KBr): $\tilde{v}=3454,3089,2933,1685,1550,1432,1400,1085,844,744$ $\mathrm{cm}^{-1}$ (Supporting Information Figure S4).

4,7-Dichloro-1,10-phenanthroline-5-carbonitrile (4e); beige; $0.6 \mathrm{~g}(2.4 \mathrm{mmol}, 48 \%) ; \mathrm{m} . \mathrm{p} .=249.7-250.0^{\circ} \mathrm{C}$; ${ }^{1} \mathrm{H}-\mathrm{NMR}\left(\mathrm{CDCl}_{3} ; 400.2 \mathrm{MHz}\right) \delta=7.84\left(\mathrm{~m}, 2 \mathrm{H}\right.$, aromatic), $8.88\left(\mathrm{~s}, 1 \mathrm{H}\right.$, aromatic), $9.13\left(\mathrm{~d},{ }^{3} J_{\mathrm{H}, \mathrm{H}}=4.7 \mathrm{~Hz}\right.$, $1 \mathrm{H}$, aromatic), $9.19\left(\mathrm{~d},{ }^{3} \mathrm{~J}_{\mathrm{H}, \mathrm{H}}=4.7 \mathrm{~Hz}, 1 \mathrm{H}\right.$, aromatic $) ;{ }^{13} \mathrm{C}\left\{{ }^{1} \mathrm{H}\right\}-\mathrm{NMR}\left(\mathrm{CDCl}_{3} ; 125.78 \mathrm{MHz}\right) \delta=108.1$, 118.1, 124.2, 125.2, 125.3, 126.5, 135.4, 142.3, 143.6, 147.5, 148.1, 151.5, 153.4; GC-MS: $\mathrm{t}_{\mathrm{r}}=10.1 \mathrm{~min},(\mathrm{EI})$ $\mathrm{M}^{+}=273.0(100 \%)$; Anal. Calcd for $\mathrm{C}_{13} \mathrm{H}_{5} \mathrm{~N}_{3} \mathrm{Cl}_{2}: \mathrm{C}, 56.96 ; \mathrm{H}, 1.84 ; \mathrm{N}, 15.33$; Found: $\mathrm{C}, 56.90 ; \mathrm{H}, 1.94 ; \mathrm{N}$, 15.29; UV-Vis (methanol; $\lambda$ [nm] $(\log \varepsilon)): 315$ (3.67), 302 (3.76), 292 (3.68), 268 (4.15), 249 (4.11), 231 (4.02), 210 (4.11); IR (KBr): $\tilde{v}=3406,3029,2224,1569,1498,1406,1084,846,798 \mathrm{~cm}^{-1}$ (Supporting Information Figure S5).

7-Chloropyrrolo [2,3,4-de][1,10]phenanthrolin-5(4H)-one (4f) beige; m.p dec. $=310-330{ }^{\circ} \mathrm{C} ;{ }^{1} \mathrm{H}-\mathrm{NMR}$ $\left(\mathrm{D}_{2} \mathrm{O} / \mathrm{KOD} ; 500.18 \mathrm{MHz}\right) \delta=6.47\left(\mathrm{~d},{ }^{3} J_{\mathrm{H}, \mathrm{H}}=6.9 \mathrm{~Hz}, 1 \mathrm{H}\right.$, aromatic $), 6.61\left(\mathrm{~d},{ }^{3} J_{\mathrm{H}, \mathrm{H}}=5.9 \mathrm{~Hz}, 1 \mathrm{H}\right.$, aromatic), $7.69\left(\mathrm{~s}, 1 \mathrm{H}\right.$, aromatic), $8.05\left(\mathrm{~d},{ }^{3} J_{\mathrm{H}, \mathrm{H}}=6.9 \mathrm{~Hz}, 1 \mathrm{H}\right.$, aromatic), $8.28\left(\mathrm{~d},{ }^{3} J_{\mathrm{H}, \mathrm{H}}=5.9 \mathrm{~Hz}, 1 \mathrm{H}\right.$, aromatic); ${ }^{1} \mathrm{H}-\mathrm{NMR}\left(\mathrm{D}_{2} \mathrm{O} / \mathrm{D}_{2} \mathrm{SO}_{4} ; 400.2 \mathrm{MHz}\right) \delta=7.29\left(\mathrm{~d},{ }^{3} J_{\mathrm{H}, \mathrm{H}}=6.8 \mathrm{~Hz}, 1 \mathrm{H}\right.$, aromatic), $7.42(\mathrm{~d}$, ${ }^{3} J_{\mathrm{H}, \mathrm{H}}=6.8 \mathrm{~Hz}, 1 \mathrm{H}$, aromatic), $8.28\left(\mathrm{~s}, 1 \mathrm{H}\right.$, aromatic), $8.78\left(\mathrm{~d},{ }^{3} J_{\mathrm{H}, \mathrm{H}}=6.8 \mathrm{~Hz}, 1 \mathrm{H}\right.$, aromatic), $8.87(\mathrm{~d}$, ${ }^{3} J_{\mathrm{H}, \mathrm{H}}=6.8 \mathrm{~Hz}, 1 \mathrm{H}$, aromatic); ${ }^{13} \mathrm{C}\left\{{ }^{1} \mathrm{H}\right\}-\mathrm{NMR}\left(\mathrm{D}_{2} \mathrm{O} / \mathrm{KOD} ; 125.78 \mathrm{MHz}\right) \delta=111.5,111.6,114.8,120.7$, $125.1,132.5,138.1,139.2,140.7,149.3,173.8,178.0,179.7$; MS-IT-TOF $[\mathrm{M}-\mathrm{H}]^{-}=254(80 \%),[\mathrm{M}+\mathrm{H}]^{+}=256$ (30\%), HRMS (IT TOF): $\mathrm{m} / \mathrm{z}$ Calcd for $\mathrm{C}_{13} \mathrm{H}_{7} \mathrm{~N}_{3} \mathrm{ClO}[\mathrm{M}+\mathrm{H}]^{+}=256.0278$, Found 256.0279; UV-Vis (methanol; $\lambda[\mathrm{nm}](\log \varepsilon)): 348$ (3.28), 334 (3.27), 278 (3.31), 245 (3.64), 221 (3.36); IR (KBr): $\tilde{v}=3086$, $1560,1522,1402,1385,1363,1026 \mathrm{~cm}^{-1}$ (Supporting Information Figure S6).

4,7-Dichloro-2,9-dimethyl-1,10-phenanthroline (4g) [22,42,44,45]; beige; $1.3 \mathrm{~g}$ (4.6 mmol, 92\%); m.p dec. $=201.0-201.5^{\circ} \mathrm{C} ;{ }^{1} \mathrm{H}-\mathrm{NMR}\left(\mathrm{CDCl}_{3} ; 400.2 \mathrm{MHz}\right) \delta=2.93\left(\mathrm{~s}, 6 \mathrm{H}, 2 \mathrm{CH}_{3}\right), 7.63(\mathrm{~s}, 2 \mathrm{H}$, aromatic), $8.24\left(\mathrm{~s}, 2 \mathrm{H}\right.$, aromatic); ${ }^{13} \mathrm{C}\left\{{ }^{1} \mathrm{H}\right\}-\mathrm{NMR}\left(\mathrm{CDCl}_{3} ; 100.5 \mathrm{MHz}\right) \delta=26.0,122.3,124.4,125.1,143.0,146.2$, 
160.1; CP/MAS ${ }^{13} \mathrm{C}-\mathrm{NMR} \delta=25.1,121.7,141.3,143.8,159.3 ; \mathrm{CP} / \mathrm{MAS}{ }^{15} \mathrm{~N}-\mathrm{NMR} \delta=-76.15 ; \mathrm{IR}(\mathrm{KBr})$ : $\tilde{v}=2963,1637,1568,1522,1402,1385,1363,1026 \mathrm{~cm}^{-1}$ (Supporting Information Figure S7).

4,7-Dichloro-5-fluoro-2,9-dimethyl-1,10-phenanthroline (4h) [44]; beige; $1.4 \mathrm{~g}$ (4.6 mmol, 93\%); m.p dec. $=213.0-213.5{ }^{\circ} \mathrm{C} ;{ }^{1} \mathrm{H}-\mathrm{NMR}\left(\mathrm{CDCl}_{3} ; 400.2 \mathrm{MHz}\right) \delta=2.97\left(\mathrm{~s}, 3 \mathrm{H}, \mathrm{CH}_{3}\right), 3.01\left(\mathrm{~s}, 3 \mathrm{H}, \mathrm{CH}_{3}\right)$, $7.67\left(\mathrm{~s}, 1 \mathrm{H}\right.$, aromatic), $7.68\left(\mathrm{~s}, 1 \mathrm{H}\right.$, aromatic), $7.86\left(\mathrm{~d},{ }^{3} \mathrm{~J}_{\mathrm{H}, \mathrm{F}}=13.1 \mathrm{~Hz}, 1 \mathrm{H}\right.$, aromatic); ${ }^{13} \mathrm{C}\left\{{ }^{1} \mathrm{H}\right\}-\mathrm{NMR}$ $\left(\mathrm{CDCl}_{3} ; 100.5 \mathrm{MHz}\right) \delta=25.3,25.5,105.6\left(\mathrm{~d},{ }^{2} J_{\mathrm{C}, \mathrm{F}}=26.0 \mathrm{~Hz}\right), 118.0\left(\mathrm{~d},{ }^{3} J_{\mathrm{C}, \mathrm{F}}=12.5 \mathrm{~Hz}\right), 125.1(\mathrm{~d}$, $\left.{ }^{3} J_{\mathrm{C}, \mathrm{F}}=11.1 \mathrm{~Hz}\right), 126.1\left(\mathrm{~d},{ }^{1} J_{\mathrm{C}, \mathrm{F}}=184.1 \mathrm{~Hz}\right), 140.3,142.8\left(\mathrm{~d},{ }^{2} J_{\mathrm{C}, \mathrm{F}}=68.6 \mathrm{~Hz}\right), 147.0,154.9,157.5,159.1$, $161.3 ;{ }^{19} \mathrm{~F}\left\{{ }^{1} \mathrm{H}\right\}-\mathrm{NMR}\left(\mathrm{CDCl}_{3} ; 470.5 \mathrm{MHz}\right) \delta=-110.97 ;{ }^{19} \mathrm{~F}-\mathrm{NMR}\left(\mathrm{CDCl}_{3} ; 470.5 \mathrm{MHz}\right) \delta=-110.97(\mathrm{~d}$, $\left.{ }^{3} J_{\mathrm{F}, \mathrm{H}}=11.5 \mathrm{~Hz}\right) ; \mathrm{GC}-\mathrm{MS}: \mathrm{t}_{\mathrm{r}}=8.2 \mathrm{~min},(\mathrm{EI}) \mathrm{M}^{+}=294(100 \%), 296 .(64 \%),[\mathrm{M}-\mathrm{Cl}]^{+}=259(12 \%) ;$ Anal. Calcd for $\mathrm{C}_{14} \mathrm{H}_{9} \mathrm{~N}_{2} \mathrm{Cl}_{2} \mathrm{~F}$ : C, 56.97; H, 3.07; N, 9.49; Found: C, 56.90; H, 3.19; N, 9.32; UV-Vis (methanol; $\lambda[\mathrm{nm}](\log \varepsilon)): 309$ (3.88), 296 (3.95), 273 (4.46), 241 (4.47), 211 (4.40); IR (KBr): $\tilde{v}=3471$, 3075, 1628, $1579,1375,1145,858 \mathrm{~cm}^{-1}$ (Supporting Information Figure S8).

4,5,7-Trichloro-2,9-dimethyl-1,10-phenanthroline (4i); beige; $1.2 \mathrm{~g}(3.9 \mathrm{mmol}, 78 \%) ; \mathrm{m} \cdot \mathrm{p}_{\mathrm{dec}}=162.9-163.7^{\circ} \mathrm{C}$; ${ }^{1} \mathrm{H}-\mathrm{NMR}\left(\mathrm{CDCl}_{3} ; 400.2 \mathrm{MHz}\right) \delta=2.92\left(\mathrm{~s}, 3 \mathrm{H}, \mathrm{CH}_{3}\right), 2.94\left(\mathrm{~s}, 3 \mathrm{H}, \mathrm{CH}_{3}\right), 7.63(\mathrm{~s}, 1 \mathrm{H}$, aromatic), $7.66(\mathrm{~s}, 1 \mathrm{H}$, aromatic), 8.27 (s, $1 \mathrm{H}$, aromatic); ${ }^{13} \mathrm{C}\left\{{ }^{1} \mathrm{H}\right\}-\mathrm{NMR}\left(\mathrm{CDCl}_{3} ; 100.5 \mathrm{MHz}\right) \delta=25.3,25.7,122.7,124.5,124.6$, 125.0, 128.0, 129.0, 142.1, 142.3, 144.8, 147.6, 160.2, 160.3; GC-MS: $\mathrm{t}_{\mathrm{r}}=9.9 \mathrm{~min},(\mathrm{EI}) \mathrm{M}^{+}=310$ (100\%), 312 $(95 \%),[\mathrm{M}-\mathrm{Cl}]^{+}=275(11 \%),[\mathrm{M}-2 \mathrm{Cl}]^{+}=240(40 \%)$; Anal. Calcd for $\mathrm{C}_{14} \mathrm{H}_{9} \mathrm{~N}_{2} \mathrm{Cl}_{3}: \mathrm{C}, 53.97 ; \mathrm{H}, 2.91 ; \mathrm{N}$, 8.99; Found: C, 53.90; H, 3.01; N, 8.87; UV-Vis (methanol; $\lambda$ [nm] (loge)): 316 (4.16), 304 (4.21), 274 (4.64), 247 (4.67), 210 (4.55); IR (KBr): $\tilde{v}=3456,3065,2922,1594,1576,1528,1332,905,874 \mathrm{~cm}^{-1}$ (Supporting Information Figure S9).

5-Bromo-4,7-dichloro-2,9-dimethyl-1,10-phenanthroline (4j); beige; $1.2 \mathrm{~g}$ (3.4 mmol, 68\%); m.p dec $=180.1-181.7{ }^{\circ} \mathrm{C} ;{ }^{1} \mathrm{H}-\mathrm{NMR}\left(\mathrm{CDCl}_{3} ; 400.2 \mathrm{MHz}\right) \delta=2.93\left(\mathrm{~s}, 3 \mathrm{H}, \mathrm{CH}_{3}\right), 2.95\left(\mathrm{~s}, 3 \mathrm{H}, \mathrm{CH}_{3}\right)$, $7.63\left(\mathrm{~s}, 1 \mathrm{H}\right.$, aromatic), $7.69\left(\mathrm{~s}, 1 \mathrm{H}\right.$, aromatic), $8.58\left(\mathrm{~s}, 1 \mathrm{H}\right.$, aromatic); ${ }^{13} \mathrm{C}\left\{{ }^{1} \mathrm{H}\right\}-\mathrm{NMR}\left(\mathrm{CDCl}_{3} ; 100.5 \mathrm{MHz}\right)$ $\delta=25.2,25.8,116.2,123.2,125.0,125.1,127.8,129.2,142.1,143.0,145.0,147.2,160.0,160.5$; GC-MS: $\mathrm{t}_{\mathrm{r}}=12.5 \mathrm{~min},(\mathrm{EI}) \mathrm{M}^{+}=354(63.7 \%), 356(100 \%), 358(44 \%),[\mathrm{M}-\mathrm{Br}]^{+}=275(28 \%)$; Anal. Calcd for $\mathrm{C}_{14} \mathrm{H}_{9} \mathrm{~N}_{2} \mathrm{Cl}_{2} \mathrm{Br}$ : C, 47.23; H, 2.55; N, 7.87; Found: C, 47.90; H, 2.67; N, 7.86; UV-Vis (methanol; $\lambda$ [nm] (loge)): 318 (3.97), 306 (4.02), 275 (4.43), 249 (4.43), 213 (4.34); IR (KBr): $\tilde{v}=3452,2923,1588,1438,1117$, $900,876,718 \mathrm{~cm}^{-1}$ (Supporting Information Figure S10).

4,7-Dichloro-2,5,9-trimethyl-1,10-phenanthroline (4k) [42,44]; beige; $1.2 \mathrm{~g}$ (4.3 mmol, 86\%); m.p. $=111.9-112.6{ }^{\circ} \mathrm{C} ;{ }^{1} \mathrm{H}-\mathrm{NMR}\left(\mathrm{CDCl}_{3} ; 400.2 \mathrm{MHz}\right) \delta=2.92\left(\mathrm{~s}, 3 \mathrm{H}, \mathrm{CH}_{3}\right), 2.96\left(\mathrm{~s}, 3 \mathrm{H}, \mathrm{CH}_{3}\right)$, $3.11\left(\mathrm{~s}, 3 \mathrm{H}, \mathrm{CH}_{3}\right), 7.62$ (bs, $2 \mathrm{H}$, aromatic), 7.95 (s, $1 \mathrm{H}$, aromatic); ${ }^{13} \mathrm{C}\left\{{ }^{1} \mathrm{H}\right\}-\mathrm{NMR}\left(\mathrm{CDCl}_{3} ; 100.5 \mathrm{MHz}\right)$ $\delta=25.2,25.6,26.3,123.9,124.5,124.6,125.4,126.9,133.8,142.3,143.1,144.9,147.2,159.0,159.1 ;$ CP/MAS ${ }^{13} \mathrm{C}-\mathrm{NMR} \delta=25.0,27.5,122.5,123.5,131.0,139.0,143.3,145.3,158.3 ;$ CP MAS ${ }^{15} \mathrm{~N}-\mathrm{NMR} \delta=-75.52 ;$ GC-MS: $t_{\mathrm{r}}=9.7 \mathrm{~min},(\mathrm{EI}) \mathrm{M}^{+}=290(100 \%),[\mathrm{M}-\mathrm{Cl}]^{+}=255(12 \%)$; Anal. Calcd for $\mathrm{C}_{15} \mathrm{H}_{12} \mathrm{~N}_{2} \mathrm{Cl}_{2}$ : C, 61.88; H, 4.15; N, 9.62; Found: C, 61.89; H, 4.20; N, 9.60; UV-Vis (methanol; $\lambda[\mathrm{nm}](\log \varepsilon)$ ): 343 (3.57), 311 (3.73), 275 (4.30), 254 (4.34), 213 (4.24); IR (KBr): $\tilde{v}=3452,2924,1609,1579,1530,1439,1380,1236$, $902,871,716 \mathrm{~cm}^{-1}$ (Supporting Information Figure S11).

4,7-Dichloro-2,9-dimethyl-1,10-phenanthroline-5-carbonitrile (41); beige; $0.5 \mathrm{~g}$ (1.6 mmol, 32\%); m.p dec $=188.6-189.9{ }^{\circ} \mathrm{C}^{1}{ }^{1} \mathrm{H}-\mathrm{NMR}\left(\mathrm{CDCl}_{3} ; 400.2 \mathrm{MHz}\right) \delta=2.96\left(\mathrm{~s}, 3 \mathrm{H}, \mathrm{CH}_{3}\right), 2.99\left(\mathrm{~s}, 3 \mathrm{H}, \mathrm{CH}_{3}\right)$, $7.72\left(\mathrm{~s}, 1 \mathrm{H}\right.$, aromatic), $7.73\left(\mathrm{~s}, 1 \mathrm{H}\right.$, aromatic), $8.79\left(\mathrm{~s}, 1 \mathrm{H}\right.$, aromatic); $\left.{ }^{13} \mathrm{C}^{1} \mathrm{H}\right\}-\mathrm{NMR}\left(\mathrm{CDCl}_{3} ; 125.78 \mathrm{MHz}\right)$ $\delta=25.6,26.2,106.9,118.4,122.6,123.7,125.6,127.0,134.3,142.2,143.7,146.1,146.8,161.6,163.8$; GC-MS: $\mathrm{t}_{\mathrm{r}}=10.5 \mathrm{~min},(\mathrm{EI}) \mathrm{M}^{+}=301(100 \%),[\mathrm{M}-\mathrm{Cl}]^{+}=266(14 \%)$; Anal. Calcd for $\mathrm{C}_{15} \mathrm{H}_{9} \mathrm{~N}_{3} \mathrm{Cl}_{2}: \mathrm{C}, 59.63 ; \mathrm{H}$, 3.00; N, 13.91; Found: C, 59.89; H, 3.27; N, 13.87; UV-Vis (methanol; $\lambda$ [nm] $(\log \varepsilon)$ ): 353 (3.07), 337 (3.68), 319 (4.24), 307 (4.31), 275 (4.67), 249 (4.60), 235 (4.54), 211 (4.59); IR (KBr): $\tilde{v}=3471,3406,2223,1587$, $1443,1357,1338,908,847 \mathrm{~cm}^{-1}$ (Supporting Information Figure S12).

Ethyl 4,7-dichloro-2,9-dimethyl-1,10-phenanthroline-5-carboxylate (4m); beige; $0.7 \mathrm{~g}$ (1.9 mmol, 38\%); m.p. $=115.9-116.2{ }^{\circ} \mathrm{C} ;{ }^{1} \mathrm{H}-\mathrm{NMR}\left(\mathrm{CDCl}_{3} ; 400.2 \mathrm{MHz}\right) \delta=1.43\left(\mathrm{t},{ }^{3} \mathrm{~J}_{\mathrm{H}, \mathrm{H}}=7.1 \mathrm{~Hz}, 3 \mathrm{H}, \mathrm{OCH}_{2} \mathrm{CH}_{3}\right), 2.93$ $\left(\mathrm{s}, 3 \mathrm{H}, \mathrm{CH}_{3}\right), 2.94\left(\mathrm{~s}, 3 \mathrm{H}, \mathrm{CH}_{3}\right), 4.49\left(\mathrm{q},{ }^{3} \mathrm{~J}_{\mathrm{H}, \mathrm{H}}=7.1 \mathrm{~Hz}, 2 \mathrm{H}, \mathrm{OCH}_{2}\right), 7.65(2 \mathrm{~s}, 2 \mathrm{H}$, aromatic), 8.31 (s, 
$1 \mathrm{H}$, aromatic); ${ }^{13} \mathrm{C}\left\{{ }^{1} \mathrm{H}\right\}-\mathrm{NMR}\left(\mathrm{CDCl}_{3} ; 100.5 \mathrm{MHz}\right) \delta=14.1,25.5,25.9,62.6,122.1,123.5,123.8,124.9$, 126.0, 129.3, 141.7, 143.6, 146.3, 146.6, 160.5, 161.5, 169.0; GC-MS: $\mathrm{t}_{\mathrm{r}}=10.9 \mathrm{~min},(\mathrm{EI}) \mathrm{M}^{+}=348(45 \%)$, [M-Cl] ${ }^{+}=313(21 \%)$; Anal. Calcd for $\mathrm{C}_{17} \mathrm{H}_{14} \mathrm{~N}_{2} \mathrm{Cl}_{2} \mathrm{O}_{2}: \mathrm{C}, 58.47 ; \mathrm{H}, 4.04 ; \mathrm{N}, 8.02$; Found: $\mathrm{C}, 58.90 ; \mathrm{H}$, 4.25; N, 7.98; UV-Vis (methanol; $\lambda$ [nm] (loge)): 308 (3.88), 295 (3.95), 272 (4.43), 245 (4.44), 212 (4.33); IR (KBr): $\tilde{v}=3432,2980,1727,1577,1275,1248,1214,1190,1122,1031 \mathrm{~cm}^{-1}$ (Supporting Information Figure S13).

\subsubsection{Hydrolyses of $4 \mathbf{e}$}

Compound $4 \mathrm{e}(1.0 \mathrm{~g}, 3.7 \mathrm{mmol})$ was dissolved in concentrated hydrochloric acid $(35 \%)(23 \mathrm{~mL})$ at r.t. After stirring for $72 \mathrm{~h}$ at $100{ }^{\circ} \mathrm{C}$, the volatiles were evaporated. The crude product was purified by extraction at Soxhlet apparatus $\left(\mathrm{H}_{2} \mathrm{O}\right)$ and finally was dried over $\mathrm{P}_{4} \mathrm{O}_{10}$ to yield a precipitate of 7-chloropyrrolo [2,3,4-de][1,10]phenanthrolin-5(4H)-one (4f, $0.3 \mathrm{~g}, 1.4 \mathrm{mmol}, 37 \%)$.

\subsection{Syntheses of 4,7-di(pyrrolidin-1-yl)-1,10-phenanthrolines $\mathbf{5 a}, \mathbf{5 b}, \mathbf{5 c}, \mathbf{5 d}$ and $\mathbf{5 e}$}

These were based on the procedure described in [24]. Our modifications to this procedure involve the purification. The appropriate compounds $4 \mathbf{a}, 4 \mathbf{c}, 4 \mathrm{~g}$, $4 \mathrm{~h}$ or $4 \mathbf{k}(1.0 \mathrm{mmol})$ were mixed with pyrrolidine $(1.5 \mathrm{~g}, 21.0 \mathrm{mmol})$ in a microvave vial equipped with a magnetic stir bar. The vial was sealed, placed in a microwave reactor and heated at $130{ }^{\circ} \mathrm{C}$ for $45 \mathrm{~min}$ (for compound $\mathbf{5 a}$ ) or $2 \mathrm{~h}$ (for compounds $\mathbf{5 b}, \mathbf{5 c}$, $\mathbf{5 d}$, and $\mathbf{5 e})$. The mixture was cooled to r.t., transferred to a round bottom flask $(100 \mathrm{~mL})$ and evaporated under reduced pressure. The residue has been washed with saturated $\mathrm{NaHCO}_{3}(2 \times 10 \mathrm{~mL})$ with stirring, and then with water $(2 \times 10 \mathrm{~mL})$. The organic residue has been dissolved with $\mathrm{CH}_{2} \mathrm{Cl}_{2}(20 \mathrm{~mL})$, dried over $\mathrm{MgSO}_{4}$ and evaporated under reduced pressure affording the corresponding products. Next, the crude products were purified by chromatography on silica gel using methanol/dichloromethane as eluent and finally crystallization from $\mathrm{CH}_{2} \mathrm{Cl}_{2}$ to yield precipitates as follows:

4,7-Di(pyrrolidin-1-yl)-1,10-phenanthroline (5a) [24,46]; $0.1 \mathrm{~g}(0.4 \mathrm{mmol}, 38 \%) ; \mathrm{m} \cdot \mathrm{p}_{\mathrm{dec}} .=246.8-247.7^{\circ} \mathrm{C}$; ${ }^{1} \mathrm{H}-\mathrm{NMR}\left(\mathrm{CDCl}_{3} ; 400.2 \mathrm{MHz}\right) \delta=2.03\left(\mathrm{~m}, 8 \mathrm{H}, 4 \mathrm{CH}_{2}\right), 3.67\left(\mathrm{~m}, 8 \mathrm{H}, 4 \mathrm{CH}_{2}\right), 6.69\left(\mathrm{~d},{ }^{3} J_{\mathrm{H}, \mathrm{H}}=5.5 \mathrm{~Hz}, 2 \mathrm{H}\right.$, aromatic), $7.93\left(\mathrm{~s}, 2 \mathrm{H}\right.$, aromatic), $8.72\left(\mathrm{~d},{ }^{3} \mathrm{~J}_{\mathrm{H}, \mathrm{H}}=5.4 \mathrm{~Hz}, 2 \mathrm{H}\right.$, aromatic); ${ }^{13} \mathrm{C}\left\{{ }^{1} \mathrm{H}\right\}-\mathrm{NMR}\left(\mathrm{CDCl}_{3} ; 100.5\right.$ MHz) $\delta=26.1,52.4,105.6,119.5,119.6,148.5,149.4,152.9$; MS-IT-TOF $[\mathrm{M}+\mathrm{H}]^{+}=319(100 \%)$, HRMS (IT TOF): $\mathrm{m} / \mathrm{z}$ Calcd for $\mathrm{C}_{20} \mathrm{H}_{23} \mathrm{~N}_{4}[\mathrm{M}+\mathrm{H}]^{+}=319.1917$, Found 319.1916; UV-Vis (methanol; $\lambda$ [nm] $(\log \varepsilon)$ ): 362 (4.26), 328 (4.18), 274 (4.48), 255 (4.43), 217 (4.54); IR (ATR): $\tilde{v}=2952,2935,2860,1662,1615,1552$, 1434,1349, 791, $729 \mathrm{~cm}^{-1}$; (Supporting Information Figure S14).

5-Chloro-4,7-di(pyrrolidin-1-yl)-1,10-phenanthroline (5b); $0.1 \mathrm{~g}(0.4 \mathrm{mmol}, 42 \%) ; \mathrm{m} . \mathrm{p} .=151.0-151.6{ }^{\circ} \mathrm{C}$; ${ }^{1} \mathrm{H}-\mathrm{NMR}\left(\mathrm{CDCl}_{3} ; 400.2 \mathrm{MHz}\right) \delta=1.99\left(\mathrm{~m}, 4 \mathrm{H}, 2 \mathrm{CH}_{2}\right), 2.07\left(\mathrm{~m}, 4 \mathrm{H}, 2 \mathrm{CH}_{2}\right), 3.53\left(\mathrm{~m}, 4 \mathrm{H}, 2 \mathrm{CH}_{2}\right), 3.70(\mathrm{~m}$, $\left.4 \mathrm{H}, 2 \mathrm{CH}_{2}\right), 6.67\left(\mathrm{~d},{ }^{3} J_{\mathrm{H}, \mathrm{H}}=5.8 \mathrm{~Hz}, 1 \mathrm{H}\right.$, aromatic), $6.90\left(\mathrm{~d},{ }^{3} J_{\mathrm{H}, \mathrm{H}}=5.6 \mathrm{~Hz}, 1 \mathrm{H}\right.$, aromatic $), 7.92(\mathrm{~s}, 1 \mathrm{H}$, aromatic), $8.73\left(\mathrm{~d},{ }^{3} J_{\mathrm{H}, \mathrm{H}}=5.7 \mathrm{~Hz}, 1 \mathrm{H}\right.$, aromatic $), 8.74\left(\mathrm{~d},{ }^{3} J_{\mathrm{H}, \mathrm{H}}=5.7 \mathrm{~Hz}, 1 \mathrm{H}\right.$, aromatic $) ;{ }^{13} \mathrm{C}\left\{{ }^{1} \mathrm{H}\right\}-\mathrm{NMR}$ $\left(\mathrm{CDCl}_{3} ; 100.5 \mathrm{MHz}\right) \delta=25.1,26.1,52.1,52.4,105.9,107.9,117.7,118.5,122.4,124.1,145.2,147.7,148.2$, 148.9, 152.7, 153.9; MS-IT-TOF [M+H] ${ }^{+}=353$ (100\%), HRMS (IT TOF): $\mathrm{m} / z$ Calcd for $\mathrm{C}_{20} \mathrm{H}_{22} \mathrm{ClN}_{4}[\mathrm{M}+$ $\mathrm{H}]^{+}=353.1527$, Found 353.1526; UV-Vis (methanol; $\lambda$ [nm] (loge)): 372 (3.98), 333 (3.88), 281 (4.22), 262 (4.19), 220 (4.65); IR (KBr): $\tilde{v}=2956,2870,1572,1514,1445,1350,803 \mathrm{~cm}^{-1}$; (Supporting Information Figure S15).

2,9-Dimethyl-4,7-di(pyrrolidin-1-yl)-1,10-phenanthroline (5c); $0.2 \mathrm{~g}(0.5 \mathrm{mmol}, 48 \%) ; \mathrm{m} . \mathrm{p} .=165.8-166.0{ }^{\circ} \mathrm{C}$; ${ }^{1} \mathrm{H}-\mathrm{NMR}\left(\mathrm{CDCl}_{3} ; 500.18 \mathrm{MHz}\right) \delta=2.04\left(\mathrm{~m}, 8 \mathrm{H}, 4 \mathrm{CH}_{2}\right), 2.77\left(\mathrm{~s}, 6 \mathrm{H}, 2 \mathrm{CH}_{3}\right), 3.68\left(\mathrm{~m}, 8 \mathrm{H}, 4 \mathrm{CH}_{2}\right), 6.61(\mathrm{~s}$, $2 \mathrm{H}$, aromatic), $7.90\left(\mathrm{~s}, 2 \mathrm{H}\right.$, aromatic); ${ }^{13} \mathrm{C}\left\{{ }^{1} \mathrm{H}\right\}-\mathrm{NMR}\left(\mathrm{CDCl}_{3} ; 100.5 \mathrm{MHz}\right) \delta=25.8,26.0,52.4,105.7,118.2$, 119.0, 146.3, 158.4, 157.5; CP/MAS ${ }^{13}$ C-NMR $\delta=26.0,51.8,104.7,117.5,147.3,149.6,154.6$; CP/MAS ${ }^{15} \mathrm{~N}-\mathrm{NMR} \delta=-291.94,-62.66$; MS-IT-TOF $[\mathrm{M}+\mathrm{H}]^{+}=347$ (100\%), HRMS (IT TOF): $\mathrm{m} / \mathrm{z}$ Calcd for $\mathrm{C}_{22} \mathrm{H}_{27} \mathrm{~N}_{4}[\mathrm{M}+\mathrm{H}]^{+}=347.2230$, Found 347.2229; UV-Vis (methanol; $\lambda$ [nm] (loge)): 387 (3.80), 358 (4.10), 322 (4.04), 273 (4.34), 245 (4.26), 214 (4.35); IR (ATR): $\tilde{v}=2954,2866,1539,1423,1349,1078,802 \mathrm{~cm}^{-1}$; (Supporting Information Figure S16). 
5-Fluoro-2,9-dimethyl-4,7-di(pyrrolidin-1-yl)-1,10-phenanthroline (5d); 0.2 g (0.5 mmol, 51\%); m.p. $=100.9-101.0{ }^{\circ} \mathrm{C} ;{ }^{1} \mathrm{H}-\mathrm{NMR}\left(\mathrm{CDCl}_{3} ; 500.18 \mathrm{MHz}\right) \delta=1.96\left(\mathrm{~m}, 4 \mathrm{H}, 2 \mathrm{CH}_{2}\right), 2.01\left(\mathrm{~m}, 4 \mathrm{H}, 2 \mathrm{CH}_{2}\right), 2.74$ $\left(\mathrm{s}, 3 \mathrm{H}, \mathrm{CH}_{3}\right), 2.76\left(\mathrm{~s}, 3 \mathrm{H}, \mathrm{CH}_{3}\right), 3.47\left(\mathrm{~m}, 4 \mathrm{H}, 2 \mathrm{CH}_{2}\right), 3.61\left(\mathrm{~m}, 4 \mathrm{H}, 2 \mathrm{CH}_{2}\right), 6.58(\mathrm{~s}, 1 \mathrm{H}$, aromatic), 6.65 (s, $1 \mathrm{H}$, aromatic), $7.45\left(\mathrm{~d},{ }^{3} J_{\mathrm{H}, \mathrm{F}}=15.5 \mathrm{~Hz}, 1 \mathrm{H}\right.$, aromatic); ${ }^{13} \mathrm{C}\left\{{ }^{1} \mathrm{H}\right\}-\mathrm{NMR}\left(\mathrm{CDCl}_{3} ; 100.5 \mathrm{MHz}\right) \delta=25.6$, 25.6, 25.8, 25.9, 25.9, 51.7, 51.8, 52.0, $102.9\left(\mathrm{~d},{ }^{1} J_{\mathrm{C}, \mathrm{F}}=27.1 \mathrm{~Hz}\right), 106.1,109.9\left(\mathrm{~d},{ }^{2} J_{\mathrm{C}, \mathrm{F}}=16.2 \mathrm{~Hz}\right), 117.6(\mathrm{~d}$, $\left.{ }^{2} J_{\mathrm{C}, \mathrm{F}}=10.6 \mathrm{~Hz}\right), 144.5,148.7\left(\mathrm{~d},{ }^{3} J_{\mathrm{C}, \mathrm{F}}=4.1 \mathrm{~Hz}\right), 152.1(\mathrm{~d}, J=1.0 \mathrm{~Hz}), 152.3,152.9\left(\mathrm{~d},{ }^{3} J_{\mathrm{C}, \mathrm{F}}=4.3 \mathrm{~Hz}\right)$, $154.8,157.1\left(\mathrm{~d},{ }^{4} J_{\mathrm{C}, \mathrm{F}}=1.6 \mathrm{~Hz}\right), 158.7 ;{ }^{19} \mathrm{~F}-\mathrm{NMR}\left(\mathrm{CDCl}_{3} ; 470.5 \mathrm{MHz}\right) \delta=-112.29\left(\mathrm{~d},{ }^{3} J_{\mathrm{F}, \mathrm{H}}=15.5 \mathrm{~Hz}\right)$; ${ }^{19} \mathrm{~F}\left\{{ }^{1} \mathrm{H}\right\}-\mathrm{NMR}\left(\mathrm{CDCl}_{3} ; 470.5 \mathrm{MHz}\right) \delta=-112.29$; MS-IT-TOF [M + H] ${ }^{+}=365(100 \%), \mathrm{HRMS}$ (IT TOF): $\mathrm{m} / \mathrm{z}$ Calcd for $\mathrm{C}_{22} \mathrm{H}_{26} \mathrm{FN}_{4}[\mathrm{M}+\mathrm{H}]^{+}=365.2136$, Found 365.2135; UV-Vis (methanol; $\lambda[\mathrm{nm}](\log \varepsilon)$ ): 407 (3.91), 359 (4.30), 325 (4.26), 281 (4.56), 256 (4.46), 221 (4.73); IR (KBr): $\tilde{v}=2957,2866,1564,1431,1352$, 1090, $820 \mathrm{~cm}^{-1}$; CCDC 1479401; (Supporting Information Figure S17).

2,5,9-Trimethyl-4,7-di(pyrrolidin-1-yl)-1,10-phenanthroline (5e); 0.1 g $\quad(0.4 \quad \mathrm{mmol}, \quad 39 \%)$; m.p. $=101.7-102.0{ }^{\circ} \mathrm{C} ;{ }^{1} \mathrm{H}-\mathrm{NMR}\left(\mathrm{CDCl}_{3} ; 400.2 \mathrm{MHz}\right) \delta=1.97\left(\mathrm{~m}, 4 \mathrm{H}, 2 \mathrm{CH}_{2}\right), 2.03(\mathrm{~m}, 4 \mathrm{H}$, $\left.2 \mathrm{CH}_{2}\right), 2.68\left(\mathrm{~s}, 3 \mathrm{H}, \mathrm{CH}_{3}\right), 2.76\left(\mathrm{~s}, 3 \mathrm{H}, \mathrm{CH}_{3}\right), 2.77\left(\mathrm{~s}, 3 \mathrm{H}, \mathrm{CH}_{3}\right), 3.35\left(\mathrm{~m}, 4 \mathrm{H}, 2 \mathrm{CH}_{2}\right), 3.68\left(\mathrm{~m}, 4 \mathrm{H}, 2 \mathrm{CH}_{2}\right)$, $6.61\left(\mathrm{~s}, 1 \mathrm{H}\right.$, aromatic), 6.85 (s, $1 \mathrm{H}$, aromatic), $7.63\left(\mathrm{~s}, 1 \mathrm{H}\right.$, aromatic); ${ }^{13} \mathrm{C}\left\{{ }^{1} \mathrm{H}\right\}-\mathrm{NMR}\left(\mathrm{CDCl}_{3} ; 100.5 \mathrm{MHz}\right)$ $\delta=21.4,24.4,25.6,25.7,26.0,52.0,52.3,105.8,109.3,117.9,120.8,122.4,127.4,145.1,146.7,153.2$, 155.4, 156.7, 157.5; MS-IT-TOF [M + H] ${ }^{+}=361(100 \%)$, HRMS (IT TOF): $m / z$ Calcd for $\mathrm{C}_{23} \mathrm{H}_{29} \mathrm{~N}_{4}$ [M + $\mathrm{H}^{+}=361.2386$, Found 361.2385; UV-Vis (methanol; $\lambda$ [nm] (loge)): 361 (4.17), 325 (4.15), 285 (4.44), 260 (4.36), 216 (4.54); IR (ATR): $\tilde{v}=2962,2846,1541,1424,1349,1077,801 \mathrm{~cm}^{-1}$; (Supporting Information Figure S18).

\subsection{Syntheses of 4,7-di(9H-carbazol-9-yl)-1,10-phenanthrolines $\mathbf{5 f}, \mathbf{5 g}$, $5 \mathbf{h}$ and $\mathbf{5 m}$}

These were based on the procedure described in the literature [25]. To s suspension of $\mathrm{NaH}(0.1 \mathrm{~g}$, $3.94 \mathrm{mmol})$ in THF $(50 \mathrm{~mL}) 9 \mathrm{H}$-carbazole $(0.5 \mathrm{~g}, 3.01 \mathrm{mmol})$ was added and stirred until evolution of $\mathrm{H}_{2}$ ceased. Reagents were stirred under reflux for $30 \mathrm{~min}$. under argon. Compounds $4 \mathbf{a}, \mathbf{4 b}, \mathbf{4} \mathbf{d}$ or $4 \mathbf{e}$ $(1.50 \mathrm{mmol})$ was then added to the reaction mixture, which was refluxed overnight. After evaporation of the solvent to give a solid, water $(20 \mathrm{~mL})$ and chloroform $(100 \mathrm{~mL})$ was added. The organic layer was separated and the aqueous layer was extracted four times with chloroform. The combined organic layers were dried over $\mathrm{MgSO}_{4}$. After solvent evaporating, the crude product was purified by column chromatography on silica gel using methanol/dichloromethane as eluent to afford a white powder, and finally crystallization from a mixture of $\mathrm{CH}_{2} \mathrm{Cl}_{2}$ and hexane to yield solids as follows:

4,7-Di(9H-carbazol-9-yl)-1,10-phenanthroline [25] (5f) white 0.7 g (1.29 mmol, 86\%); m.p dec $=275.1-280.0{ }^{\circ} \mathrm{C} ;{ }^{1} \mathrm{H}-\mathrm{NMR}\left(\mathrm{CDCl}_{3} ; 500.2 \mathrm{MHz}\right) \delta=7.06\left(\mathrm{~d},{ }^{3} \mathrm{~J}_{\mathrm{H}, \mathrm{H}}=7.9 \mathrm{~Hz}, 4 \mathrm{H}\right.$, aromatic), $7.28-7.36\left(\mathrm{~m}, 10 \mathrm{H}\right.$, aromatic), $7.85\left(\mathrm{~d},{ }^{3} J_{\mathrm{H}, \mathrm{H}}=4.7 \mathrm{~Hz}, 2 \mathrm{H}\right.$, aromatic), $8.15\left(\mathrm{~d},{ }^{3} J_{\mathrm{H}, \mathrm{H}}=7.2 \mathrm{~Hz}\right.$, $4 \mathrm{H}$, aromatic), $9.49\left(\mathrm{~d},{ }^{3} \mathrm{~J}_{\mathrm{H}, \mathrm{H}}=4.7 \mathrm{~Hz}, 2 \mathrm{H}\right.$, aromatic); ${ }^{13} \mathrm{C}\left\{{ }^{1} \mathrm{H}\right\}-\mathrm{NMR}\left(\mathrm{CDCl}_{3} ; 125.78 \mathrm{MHz}\right) \delta=110.1$, 120.7, 121.0, 123.0, 124.1, 126.5, 126.7, 141.3, 143.5, 148.8, 151.6; CP/MAS ${ }^{13} \mathrm{C}-\mathrm{NMR} \delta=109.4,120.2,122.0$, 123.6, 124.8, 127.4, 139.4, 141.2, 147.5, 149.2, 150.3; CP/MAS ${ }^{15} \mathrm{~N}-\mathrm{NMR} \delta=-250.68,-73.05$; UV-Vis (methanol; $\lambda[\mathrm{nm}](\log \varepsilon)): 353$ (3.10), 332 (3.54), 319 (3.54), 277 (4.03), 268 (4.06), 247 (4.16), 230 (4.50), 210 (4.18); IR (ATR): $\tilde{v}=3062,2371,1448,1222,750,725 \mathrm{~cm}^{-1}$; (Supporting Information Figure S19).

4,7-Di(9H-carbazol-9-yl)-5-fluoro-1,10-phenanthroline (5g) $0.4 \mathrm{~g}(0.81 \mathrm{mmol}, 54 \%) ; \mathrm{m} \cdot \mathrm{p}_{\mathrm{dec}}=184.3-185.0^{\circ} \mathrm{C}$; ${ }^{1} \mathrm{H}-\mathrm{NMR}\left(\mathrm{CDCl}_{3} ; 400.2 \mathrm{MHz}\right) \delta=7.03\left(\mathrm{~d},{ }^{3} \mathrm{~J}_{\mathrm{H}, \mathrm{F}}=12.6 \mathrm{~Hz}, 1 \mathrm{H}\right.$, aromatic), $7.09\left(\mathrm{dd},{ }^{3} J_{\mathrm{H}, \mathrm{H}}=7.7 \mathrm{~Hz}\right.$, ${ }^{3} J_{\mathrm{H}, \mathrm{H}}=5.2 \mathrm{~Hz}, 4 \mathrm{H}$, aromatic), 7.30-7.43 (m, 8H, aromatic), $7.91\left(\mathrm{dd},{ }^{3} J_{\mathrm{H}, \mathrm{H}}=9.9 \mathrm{~Hz},{ }^{3} J_{\mathrm{H}, \mathrm{H}}=4.7 \mathrm{~Hz}\right.$, $2 \mathrm{H}$, aromatic), $8.18\left(\mathrm{t},{ }^{3} J_{\mathrm{H}, \mathrm{H}}=8.4 \mathrm{~Hz}, 4 \mathrm{H}\right.$, aromatic), $9.47\left(\mathrm{~d},{ }^{3} J_{\mathrm{H}, \mathrm{H}}=4.4 \mathrm{~Hz}, 1 \mathrm{H}\right.$, aromatic), $9.54(\mathrm{~d}$, ${ }^{3} J_{\mathrm{H}, \mathrm{H}}=4.6 \mathrm{~Hz}, 1 \mathrm{H}$, aromatic); ${ }^{13} \mathrm{C}\left\{{ }^{1} \mathrm{H}\right\}-\mathrm{NMR}\left(\mathrm{CDCl}_{3} ; 100.5 \mathrm{MHz}\right) \delta=106.5\left(\mathrm{~d},{ }^{1} J_{\mathrm{C}, \mathrm{F}}=24.7 \mathrm{~Hz}\right), 109.4$, $110.0,119.6,120.7,120.8\left(\mathrm{~d},{ }^{4} J_{\mathrm{C}, \mathrm{F}}=1.0 \mathrm{~Hz}\right), 121.0,121.2,121.4,123.6,124.1\left(\mathrm{~d},{ }^{2} J_{\mathrm{C}, \mathrm{F}}=17.7 \mathrm{~Hz}\right), 125.1$, $125.5,126.56\left(\mathrm{~d},{ }^{3} J_{\mathrm{C}, \mathrm{F}}=4.8 \mathrm{~Hz}\right), 126.58\left(\mathrm{~d},{ }^{2} J_{\mathrm{C}, \mathrm{F}}=23.6 \mathrm{~Hz}\right), 141.1,141.6,141.7\left(\mathrm{~d},{ }^{4} J_{\mathrm{C}, \mathrm{F}}=1.9 \mathrm{~Hz}\right), 143.2$ $\left(\mathrm{d},{ }^{3} J_{\mathrm{C}, \mathrm{F}}=6.0 \mathrm{~Hz}\right), 146.1,150.0\left(\mathrm{~d},{ }^{4} J_{\mathrm{C}, \mathrm{F}}=2.5 \mathrm{~Hz}\right), 150.9,152.6,154.2,156.8 ;{ }^{19} \mathrm{~F}-\mathrm{NMR}\left(\mathrm{CDCl}_{3} ; 470.5\right.$ $\mathrm{MHz}) \delta=-112.43\left(\mathrm{~d},{ }^{3} J_{\mathrm{F}, \mathrm{H}}=10.6 \mathrm{~Hz}\right) ;{ }^{19} \mathrm{~F}\left\{{ }^{1} \mathrm{H}\right\}-\mathrm{NMR}\left(\mathrm{CDCl}_{3} ; 470.5 \mathrm{MHz}\right) \delta=-112.43$; MS-IT-TOF $[\mathrm{M}+\mathrm{H}]^{+}=529(100 \%)$, HRMS (IT TOF): $m / z$ Calcd for $\mathrm{C}_{36} \mathrm{H}_{22} \mathrm{FN}_{4}[\mathrm{M}+\mathrm{H}]^{+}=529.1828$, Found 529.1823. 
UV-Vis (methanol; $\lambda$ [nm] (loge)): 367 (3.14), 329 (3.37), 318 (3.37), 278 (3.88), 256 (3.88), 230 (4.33), 209 (4.08); IR (ATR): $\tilde{v}=3045,3023,2361,1445,1224,744,720 \mathrm{~cm}^{-1}$; (Supporting Information Figure S20).

4,7-Di(9H-carbazol-9-yl)-5-methyl-1,10-phenanthroline (5h) white $0.7 \mathrm{~g}(1.34 \mathrm{mmol}, 89 \%)$; m.p.ec.$>350{ }^{\circ} \mathrm{C}$; ${ }^{1} \mathrm{H}-\mathrm{NMR}\left(\mathrm{CDCl}_{3} ; 500.2 \mathrm{MHz}\right) \delta=1.60\left(\mathrm{~d},{ }^{4} J_{\mathrm{H}, \mathrm{H}}=0.8 \mathrm{~Hz}, 3 \mathrm{H}, \mathrm{CH}_{3}\right), 6.93\left(\mathrm{~d},{ }^{3} J_{\mathrm{H}, \mathrm{H}}=8.1 \mathrm{~Hz}, 2 \mathrm{H}\right.$, aromatic), $7.10\left(\mathrm{~d},{ }^{3} J_{\mathrm{H}, \mathrm{H}}=8.1 \mathrm{~Hz}, 2 \mathrm{H}\right.$, aromatic), $7.22\left(\mathrm{~d},{ }^{4} J_{\mathrm{H}, \mathrm{H}}=1.0 \mathrm{~Hz}, 1 \mathrm{H}\right.$, aromatic), 7.29-7.40 (m, $8 \mathrm{H}$, aromatic), $7.68\left(\mathrm{~d},{ }^{3} J_{\mathrm{H}, \mathrm{H}}=4.6 \mathrm{~Hz}, 1 \mathrm{H}\right.$, aromatic), $7.80\left(\mathrm{~d},{ }^{3} J_{\mathrm{H}, \mathrm{H}}=4.7 \mathrm{~Hz}, 1 \mathrm{H}\right.$, aromatic), $8.16(\mathrm{~d}$, ${ }^{3} J_{\mathrm{H}, \mathrm{H}}=7.7 \mathrm{~Hz}, 2 \mathrm{H}$, aromatic), $8.18\left(\mathrm{~d},{ }^{3} J_{\mathrm{H}, \mathrm{H}}=8.1 \mathrm{~Hz}, 2 \mathrm{H}\right.$, aromatic), $9.43\left(\mathrm{dd},{ }^{3} J_{\mathrm{H}, \mathrm{H}}=6.9 \mathrm{~Hz},{ }^{3} J_{\mathrm{H}, \mathrm{H}}=4.7\right.$ $\mathrm{Hz}, 2 \mathrm{H}$, aromatic); ${ }^{13} \mathrm{C}\left\{{ }^{1} \mathrm{H}\right\}-\mathrm{NMR}\left(\mathrm{CDCl}_{3} ; 100.5 \mathrm{MHz}\right) \delta=21.1,109.7,110.1,120.70,120.73,120.8,121.0$, 123.1, 123.7, 124.0, 124.5, 125.6, 126.2, 126.5, 126.8, 128.2, 133.6, 141.4, 142.2, 142.4, 143.4, 148.0, 149.7, 150.9, 151.2; CP/MAS ${ }^{13} \mathrm{C}-\mathrm{NMR} \delta=23.4,111.3,116.6,120.7,123.3,126.7,128.2,133.7,136.6,142.0,147.4$, 148.5, 150.3; CP/MAS ${ }^{15} \mathrm{~N}-\mathrm{NMR} \delta=-254.60,-249.48,-78.17,-54.38$; MS-IT-TOF $[\mathrm{M}+\mathrm{H}]^{+}=525(30 \%)$, HRMS (IT TOF): $m / z$ Calcd for $\mathrm{C}_{37} \mathrm{H}_{25} \mathrm{~N}_{4}[\mathrm{M}+\mathrm{H}]^{+}=525.2001$, Found 525.2091; UV-Vis (methanol; $\lambda$ [nm] (loge)): 355 (3.32), 333 (3.83), 319 (3.76), 277 (4.28), 256 (4.25), 245 (4.41), 232 (4.72), 210 (4.38); IR (ATR): $\tilde{v}=3046,2363,1449,1230,747,723 \mathrm{~cm}^{-1}$; (Supporting Information Figure S21).

4,7-Di(9H-carbazol-9-yl)-1,10-phenanthroline-5-carbonitrile $(5 \mathrm{~m})$ brown $0.7 \mathrm{~g}$ (1.29 mmol, 86\%); m.p dec $\cdot=145-147{ }^{\circ} \mathrm{C} ;{ }^{1} \mathrm{H}-\mathrm{NMR}\left(\mathrm{CDCl}_{3} ; 400.2 \mathrm{MHz}\right) \delta=6.93\left(\mathrm{~d},{ }^{3} J_{\mathrm{H}, \mathrm{H}}=7.4 \mathrm{~Hz}, 2 \mathrm{H}\right.$, aromatic), $7.07\left(\mathrm{~d},{ }^{3} J_{\mathrm{H}, \mathrm{H}}=7.3 \mathrm{~Hz}, 2 \mathrm{H}\right.$, aromatic), 7.31-7.43 (m, 8H, aromatic), $7.88\left(\mathrm{~d},{ }^{3} \mathrm{~J}_{\mathrm{H}, \mathrm{H}}=4.6 \mathrm{~Hz}, 1 \mathrm{H}\right.$, aromatic), $7.93\left(\mathrm{~d},{ }^{3} \mathrm{~J}_{\mathrm{H}, \mathrm{H}}=4.7 \mathrm{~Hz}, 1 \mathrm{H}\right.$, aromatic), $8.05\left(\mathrm{~s}, 1 \mathrm{H}\right.$, aromatic), $8.18\left(\mathrm{~d},{ }^{3} J_{\mathrm{H}, \mathrm{H}}=7.3 \mathrm{~Hz}, 4 \mathrm{H}\right.$, aromatic), $9.55\left(\mathrm{~d},{ }^{3} \mathrm{~J}_{\mathrm{H}, \mathrm{H}}=4.6 \mathrm{~Hz}, 1 \mathrm{H}\right.$, aromatic), $9.60\left(\mathrm{~d},{ }^{3} \mathrm{~J}_{\mathrm{H}, \mathrm{H}}=4.7 \mathrm{~Hz}, 1 \mathrm{H}\right.$, aromatic $) ;{ }^{13} \mathrm{C}\left\{{ }^{1} \mathrm{H}\right\}-\mathrm{NMR}\left(\mathrm{CDCl}_{3}\right.$; $100.5 \mathrm{MHz}) \delta=107.1,109.2,109.6,115.7,121.0,121.1,121.3,121.7,123.8,124.4,124.5,125.2,125.6,125.8$, $126.6,126.9,134.4,141.2,142.3,143.1,144.5,148.8,149.6,153.1,154.7$; MS-IT-TOF [M+H] ${ }^{+}=536(100 \%)$, HRMS (IT TOF): $m / z$ Calcd for $\mathrm{C}_{37} \mathrm{H}_{22} \mathrm{~N}_{5}[\mathrm{M}+\mathrm{H}]^{+}=536.1875$, Found 536.1876; UV-Vis (methanol; $\lambda$ [nm] (loge)): 368 (3.28), 323 (3.77), 292 (4.23), 286 (4.22), 257 (4.33), 232 (4.73); IR (ATR): $\tilde{v}=3050,2925$, $2224,1450,1226,752,726 \mathrm{~cm}^{-1}$. (Supporting Information Figure S26).

\subsection{Syntheses of 4,7-di(10H-phenothiazin-10-yl)-1,10-phenanthrolines $5 \mathbf{i}, \mathbf{5 j}, \mathbf{5 k}$ and $\mathbf{5 n}$}

To the suspension of $\mathrm{NaH}(1.1 \mathrm{~g}, 48.1 \mathrm{mmol})$ in THF $(50 \mathrm{~mL}) 10 \mathrm{H}$-phenothiazine $(8.0 \mathrm{~g}, 40.0 \mathrm{mmol})$ was added and stirred until evolution of $\mathrm{H}_{2}$ ceased. Reagents were stirred under reflux for $30 \mathrm{~min}$. under argon. Then compounds $4 \mathbf{a}, 4 \mathbf{b}, 4 \mathbf{d}$ or $4 \mathbf{e}(20.0 \mathrm{mmol})$ were added to the reaction mixture in one portion. After heating under reflux for $24 \mathrm{~h}$, the solvent was evaporated and a mixture composed of $\mathrm{CH}_{2} \mathrm{Cl}_{2}(20 \mathrm{~mL})$ and water $(20 \mathrm{~mL})$ was added. The organic layer was separated and the aqueous layer was extracted four times with chloroform $(4 \times 20 \mathrm{~mL})$. The combined organic layers were dried over $\mathrm{MgSO}_{4}$, and the crude product was purified by crystallization from mixture of $\mathrm{THF}\left(\mathrm{CH}_{2} \mathrm{Cl}_{2}\right)$ and hexane to yield solid as follows:

4,7-Di(10H-phenothiazin-10-yl)-1,10-phenanthroline (5i) greenish 10.8 g (18.8 mmol, 94\%); m.p $\mathrm{pdec}_{\text {. }}=149.4-150.0{ }^{\circ} \mathrm{C} ;{ }^{1} \mathrm{H}-\mathrm{NMR}\left(\mathrm{CDCl}_{3} ; 500.2 \mathrm{MHz}\right) \delta=6.10\left(\mathrm{dd},{ }^{3} J_{\mathrm{H}, \mathrm{H}}=8.2 \mathrm{~Hz},{ }^{4} \mathrm{~J}_{\mathrm{H}, \mathrm{H}}=1.1\right.$ $\mathrm{Hz}, 4 \mathrm{H}$, aromatic), $6.76\left(\mathrm{dt},{ }^{3} J_{\mathrm{H}, \mathrm{H}}=7.8 \mathrm{~Hz},{ }^{4} J_{\mathrm{H}, \mathrm{H}}=1.6 \mathrm{~Hz}, 4 \mathrm{H}\right.$, aromatic), $6.82\left(\mathrm{td},{ }^{3} J_{\mathrm{H}, \mathrm{H}}=7.5 \mathrm{~Hz}\right.$, ${ }^{4} J_{\mathrm{H}, \mathrm{H}}=1.2 \mathrm{~Hz}, 4 \mathrm{H}$, aromatic), $7.07\left(\mathrm{dd},{ }^{3} J_{\mathrm{H}, \mathrm{H}}=7.6 \mathrm{~Hz},{ }^{4} \mathrm{~J}_{\mathrm{H}, \mathrm{H}}=1.5 \mathrm{~Hz}, 4 \mathrm{H}\right.$, aromatic), $7.88\left(\mathrm{~d},{ }^{3} J_{\mathrm{H}, \mathrm{H}}=4.7\right.$ $\mathrm{Hz}, 2 \mathrm{H}$, aromatic), 8.15 (s, $2 \mathrm{H}$, aromatic), $9.50\left(\mathrm{~d},{ }^{3} \mathrm{~J}_{\mathrm{H}, \mathrm{H}}=4.7 \mathrm{~Hz}, 2 \mathrm{H}\right.$, aromatic); ${ }^{13} \mathrm{C}\left\{{ }^{1} \mathrm{H}\right\}-\mathrm{NMR}\left(\mathrm{CDCl}_{3}\right.$; $125.78 \mathrm{MHz}) \delta=116.0,121.1,123.4,123.5,127.1,127.3,128.5,142.9,146.5,149.5,152.0$; MS-IT-TOF [M + $\mathrm{H}]^{+}=575$ (100\%), HRMS (IT TOF): $m / z$ Calcd for $\mathrm{C}_{36} \mathrm{H}_{23} \mathrm{~N}_{4} \mathrm{~S}_{2}[\mathrm{M}+\mathrm{H}]^{+}=575.1364$, Found 575.1358. UV-Vis (methanol; $\lambda$ [nm] (loge)): 302 (3.56), 270 (4.09), 251 (4.33), 237 (4.21), 228 (4.15), 208 (4.14); IR (ATR): $\tilde{v}=3055,2984,1460,1305,1234,748,734,632 \mathrm{~cm}^{-1}$; (Supporting Information Figure S22).

5-Fluoro-4,7-di(10H-phenothiazin-10-yl)-1,10-phenanthroline (5j) brown $11.4 \mathrm{~g}$ (19.2 mmol, 96\%); m.p dec $=186.1-187.0{ }^{\circ} \mathrm{C} ;{ }^{1} \mathrm{H}-\mathrm{NMR}\left(\mathrm{CDCl}_{3} ; 500.2 \mathrm{MHz}\right) \delta=5.84\left(\mathrm{~d},{ }^{3} J_{\mathrm{H}, \mathrm{H}}=8.2 \mathrm{~Hz},{ }^{4} J_{\mathrm{H}, \mathrm{H}}=0.8\right.$ $\mathrm{Hz}, 2 \mathrm{H}$, aromatic), $6.17\left(\mathrm{~d},{ }^{3} J_{\mathrm{H}, \mathrm{H}}=8.1 \mathrm{~Hz},{ }^{4} J_{\mathrm{H}, \mathrm{H}}=1.2 \mathrm{~Hz}, 2 \mathrm{H}\right.$, aromatic), 6.69 (td, ${ }^{3} J_{\mathrm{H}, \mathrm{H}}=7.4 \mathrm{~Hz}$, ${ }^{4} J_{\mathrm{H}, \mathrm{H}}=1.6 \mathrm{~Hz}, 2 \mathrm{H}$, aromatic), $6.75\left(\mathrm{td},{ }^{3} J_{\mathrm{H}, \mathrm{H}}=7.5 \mathrm{~Hz},{ }^{4} J_{\mathrm{H}, \mathrm{H}}=1.3 \mathrm{~Hz}, 2 \mathrm{H}\right.$, aromatic), $6.80\left(\mathrm{td},{ }^{3} J_{\mathrm{H}, \mathrm{H}}=7.4\right.$ $\mathrm{Hz},{ }^{4} J_{\mathrm{H}, \mathrm{H}}=1.7 \mathrm{~Hz}, 2 \mathrm{H}$, aromatic), $6.85\left(\mathrm{td},{ }^{3} J_{\mathrm{H}, \mathrm{H}}=7.4 \mathrm{~Hz},{ }^{4} J_{\mathrm{H}, \mathrm{H}}=1.3 \mathrm{~Hz}, 2 \mathrm{H}\right.$, aromatic), 6.98 (dd, 
${ }^{3} J_{\mathrm{H}, \mathrm{H}}=7.6 \mathrm{~Hz},{ }^{4} J_{\mathrm{H}, \mathrm{H}}=1.5 \mathrm{~Hz}, 2 \mathrm{H}$, aromatic), $7.08\left(\mathrm{dd},{ }^{3} J_{\mathrm{H}, \mathrm{H}}=7.5 \mathrm{~Hz},{ }^{4} J_{\mathrm{H}, \mathrm{H}}=1.6 \mathrm{~Hz}, 2 \mathrm{H}\right.$, aromatic), $7.82\left(\mathrm{~d},{ }^{3} \mathrm{~J}_{\mathrm{H}, \mathrm{H}}=4.6 \mathrm{~Hz}, 1 \mathrm{H}\right.$, aromatic), $7.87\left(\mathrm{~d},{ }^{3} \mathrm{~J}_{\mathrm{H}, \mathrm{F}}=12.5 \mathrm{~Hz}, 1 \mathrm{H}\right.$, aromatic), $7.92\left(\mathrm{~d},{ }^{3} J_{\mathrm{H}, \mathrm{H}}=4.6 \mathrm{~Hz}, 1 \mathrm{H}\right.$, aromatic), $9.47\left(\mathrm{~d},{ }^{3} \mathrm{~J}_{\mathrm{H}, \mathrm{H}}=4.6 \mathrm{~Hz}, 1 \mathrm{H}\right.$, aromatic), $9.52\left(\mathrm{~d},{ }^{3} \mathrm{~J}_{\mathrm{H}, \mathrm{H}}=4.6 \mathrm{~Hz}, 1 \mathrm{H}\right.$, aromatic); ${ }^{13} \mathrm{C}\left\{{ }^{1} \mathrm{H}\right\}-\mathrm{NMR}$ $\left(\mathrm{CDCl}_{3} ; 125.78 \mathrm{MHz}\right) \delta=106.5\left(\mathrm{~d},{ }^{3} J_{\mathrm{C}, \mathrm{F}}=24.7 \mathrm{~Hz}\right), 115.4\left(\mathrm{~d},{ }^{1} J_{\mathrm{C}, \mathrm{F}}=108.4 \mathrm{~Hz}\right), 119.8,121.4,122.4(\mathrm{~d}$, $\left.{ }^{4} J_{\mathrm{C}, \mathrm{F}}=13.5 \mathrm{~Hz}\right), 123.3\left(\mathrm{~d},{ }^{2} J_{\mathrm{C}, \mathrm{F}}=91.9 \mathrm{~Hz}\right), 127.0\left(\mathrm{~d},{ }^{4} J_{\mathrm{C}, \mathrm{F}}=14.5 \mathrm{~Hz}\right), 127.3\left(\mathrm{~d},{ }^{3} J_{\mathrm{C}, \mathrm{F}}=16.0 \mathrm{~Hz}\right), 128.2$, $128.4\left(\mathrm{~d},{ }^{2} J_{\mathrm{C}, \mathrm{F}}=66.8 \mathrm{~Hz}\right), 142.8\left(\mathrm{~d},{ }^{5} J_{\mathrm{C}, \mathrm{F}}=11.9 \mathrm{~Hz}\right), 144.3,145.7\left(\mathrm{~d},{ }^{5} J_{\mathrm{C}, \mathrm{F}}=5.3 \mathrm{~Hz}\right), 147.3,150.6,151.1$, 153.9, 154.7, $156.8 ;{ }^{19} \mathrm{~F}-\mathrm{NMR}\left(\mathrm{CDCl}_{3} ; 470.5 \mathrm{MHz}\right) \delta=-113.33\left(\mathrm{~d},{ }^{3} \mathrm{~J}_{\mathrm{F}, \mathrm{H}}=11.9 \mathrm{~Hz}\right) ;{ }^{19} \mathrm{~F}\left\{{ }^{1} \mathrm{H}\right\}-\mathrm{NMR}\left(\mathrm{CDCl}_{3} ;\right.$ $470.5 \mathrm{MHz}) \delta=-113.33$; MS-IT-TOF [M + Na] ${ }^{+}=615(30 \%)$, HRMS (IT TOF): $\mathrm{m} / z$ Calcd for $\mathrm{C}_{20} \mathrm{H}_{23} \mathrm{~N}_{4}$ $[\mathrm{M}+\mathrm{Na}]^{+}=615.1089$, Found 615.1083. UV-Vis (methanol; $\left.\lambda[\mathrm{nm}](\log \varepsilon)\right): 307$ (3.56), $271(4.04), 250$ (4.33), 239 (4.23), 227 (4.14), 210 (4.19); IR (ATR): $\tilde{v}=3057,2962,2360,1459,1312,1235,739,643 \mathrm{~cm}^{-1}$; (Supporting Information Figure S23).

5-Methyl-4,7-di(10H-phenothiazin-10-yl)-1,10-phenanthroline (5k) purple $9.9 \mathrm{~g}(16.8 \mathrm{mmol}, 84 \%)$; m.p dec. $>350{ }^{\circ} \mathrm{C} ;{ }^{1} \mathrm{H}-\mathrm{NMR}\left(\mathrm{CDCl}_{3} ; 500.2 \mathrm{MHz}\right) \delta=2.91\left(\mathrm{~s}, 3 \mathrm{H}, \mathrm{CH}_{3}\right), 5.80\left(\mathrm{~d},{ }^{3} \mathrm{~J}_{\mathrm{H}, \mathrm{H}}=8.0 \mathrm{~Hz}, 2 \mathrm{H}\right.$, aromatic), 6.11 $\left(\mathrm{d},{ }^{3} \mathrm{~J}_{\mathrm{H}, \mathrm{H}}=7.9 \mathrm{~Hz}, 2 \mathrm{H}\right.$, aromatic), $6.65-6.85\left(\mathrm{~m}, 8 \mathrm{H}\right.$, aromatic), $6.96\left(\mathrm{~d},{ }^{3} \mathrm{~J}_{\mathrm{H}, \mathrm{H}}=7.3 \mathrm{~Hz}, 2 \mathrm{H}\right.$, aromatic), $7.07\left(\mathrm{~d},{ }^{3} J_{\mathrm{H}, \mathrm{H}}=7.4 \mathrm{~Hz}, 2 \mathrm{H}\right.$, aromatic), $7.70\left(\mathrm{~d},{ }^{3} J_{\mathrm{H}, \mathrm{H}}=4.5 \mathrm{~Hz}, 1 \mathrm{H}\right.$, aromatic), $7.83\left(\mathrm{~d},{ }^{3} J_{\mathrm{H}, \mathrm{H}}=4.5\right.$ $\mathrm{Hz}, 1 \mathrm{H}$, aromatic), $7.92\left(\mathrm{~s}, 1 \mathrm{H}\right.$, aromatic), $9.45\left(\mathrm{dd},{ }^{3} \mathrm{~J}_{\mathrm{H}, \mathrm{H}}=8.4 \mathrm{~Hz},{ }^{3} J_{\mathrm{H}, \mathrm{H}}=4.6 \mathrm{~Hz}, 2 \mathrm{H}\right.$, aromatic); ${ }^{13} \mathrm{C}\left\{{ }^{1} \mathrm{H}\right\}-\mathrm{NMR}\left(\mathrm{CDCl}_{3} ; 100.5 \mathrm{MHz}\right) \delta=23.6,115.6,116.0,119.0,120.9,123.0,123.3,124.2,126.8,127.06$, 127.1, 127.2, 127.8, 129.0, 129.4, 134.5, 142.6, 142.8, 145.4, 146.4, 148.6, 151.0, 151.3, 151.7; CP/MAS ${ }^{13} \mathrm{C}-\mathrm{NMR} \delta=25.9,116.8,123.4,124.6,127.2,135.3,141.5,144.8,147.1,149.0,152.6,153.5,155.6 ; \mathrm{CP} / \mathrm{MAS}$ ${ }^{15} \mathrm{~N}-\mathrm{NMR} \delta=-273.57,-62.21,-51.07$; MS-IT-TOF $[\mathrm{M}+\mathrm{H}]^{+}=589(100 \%)$, HRMS (IT TOF): $\mathrm{m} / z$ Calcd for $\mathrm{C}_{37} \mathrm{H}_{25} \mathrm{~N}_{4} \mathrm{~S}_{2}[\mathrm{M}+\mathrm{H}]^{+}=589.15207$, Found 589.15059; UV-Vis (methanol; $\lambda$ [nm] $(\log \varepsilon)$ ): 332 (3.28), 307 (3.79), 273 (4.29), 255 (4.58), 242 (4.48); IR (ATR): $\tilde{v}=3059,2960,1461,1307,1241,736,631 \mathrm{~cm}^{-1}$; (Supporting Information Figure S24).

4,7-Di(10H-phenothiazin-10-yl)-1,10-phenanthroline-5-carbonitrile (5n) brown $9.9 \mathrm{~g}$ (16.6 mmol, 83\%); m.p pec $=270-275{ }^{\circ} \mathrm{C} ;{ }^{1} \mathrm{H}-\mathrm{NMR}\left(\mathrm{CDCl}_{3} ; 500.2 \mathrm{MHz}\right) \delta=5.67\left(\mathrm{~d},{ }^{3} \mathrm{~J}_{\mathrm{H}, \mathrm{H}}=8.2 \mathrm{~Hz}, 2 \mathrm{H}\right.$, aromatic $), 6.10$ $\left(\mathrm{d},{ }^{3} J_{\mathrm{H}, \mathrm{H}}=8.1 \mathrm{~Hz}, 2 \mathrm{H}\right.$, aromatic), $6.67\left(\mathrm{t},{ }^{3} J_{\mathrm{H}, \mathrm{H}}=7.8 \mathrm{~Hz}, 2 \mathrm{H}\right.$, aromatic), $6.77\left(\mathrm{t},{ }^{3} J_{\mathrm{H}, \mathrm{H}}=7.4 \mathrm{~Hz}, 2 \mathrm{H}\right.$, aromatic), $6.82\left(\mathrm{t},{ }^{3} J_{\mathrm{H}, \mathrm{H}}=7.8 \mathrm{~Hz}, 2 \mathrm{H}\right.$, aromatic), $6.90\left(\mathrm{t},{ }^{3} J_{\mathrm{H}, \mathrm{H}}=7.2 \mathrm{~Hz}, 2 \mathrm{H}\right.$, aromatic), $6.98\left(\mathrm{~d},{ }^{3} J_{\mathrm{H}, \mathrm{H}}=7.6\right.$ $\mathrm{Hz}, 2 \mathrm{H}$, aromatic), $7.13\left(\mathrm{~d},{ }^{3} J_{\mathrm{H}, \mathrm{H}}=7.6 \mathrm{~Hz}, 2 \mathrm{H}\right.$, aromatic), $7.89\left(\mathrm{~d},{ }^{3} J_{\mathrm{H}, \mathrm{H}}=4.6 \mathrm{~Hz}, 1 \mathrm{H}\right.$, aromatic), 8.01 $\left(\mathrm{d},{ }^{3} J_{\mathrm{H}, \mathrm{H}}=4.6 \mathrm{~Hz}, 1 \mathrm{H}\right.$, aromatic), $8.76\left(\mathrm{~s}, 1 \mathrm{H}\right.$, aromatic), $9.58\left(\mathrm{~d},{ }^{3} J_{\mathrm{H}, \mathrm{H}}=4.6 \mathrm{~Hz}, 1 \mathrm{H}\right.$, aromatic), 9.65 $\left(\mathrm{d},{ }^{3} J_{\mathrm{H}, \mathrm{H}}=4.6 \mathrm{~Hz}, 1 \mathrm{H}\right.$, aromatic); ${ }^{13} \mathrm{C}\left\{{ }^{1} \mathrm{H}\right\}-\mathrm{NMR}\left(\mathrm{CDCl}_{3} ; 125.78 \mathrm{MHz}\right) \delta=107.5,115.1,115.9,117.6$, 119.6, 121.7, 123.4, 124.1, 126.9, 127.1, 127.3, 127.7, 128.6, 129.2, 135.0, 142.1, 142.8, 146.4, 147.4, 149.8, 150.7, 154.3, 155.1; MS-IT-TOF [M + H] $]^{+}=600(100 \%)$, HRMS (IT TOF): $m / z$ Calcd for $\mathrm{C}_{37} \mathrm{H}_{22} \mathrm{~N}_{5} \mathrm{~S}_{2}[\mathrm{M}+$ $\mathrm{H}^{+}=600.1317$, Found 600.1323; UV-Vis (methanol; $\lambda$ [nm] (loge)): 332 (3.59), 313 (3.79), 298 (3.87), 272 (4.18), 249 (4.47), 206 (4.35); IR (ATR): $\tilde{v}=3053,2926,2215,1466,1313,1239,742,633 \mathrm{~cm}^{-1}$; (Supporting Information Figure S25).

1,10-Phenanthrolin-5-amine (51) CP/MAS ${ }^{13} \mathrm{C}-\mathrm{NMR} \delta=101.4,122.9,130.5,132.4,140.8,142.9,145.4,146.5$, 152.7; CP/MAS ${ }^{15} \mathrm{~N}$ NMR $\delta=-317.08,-78.63,-75.62$; (Supporting Information Figure S27).

3.7. Synthesis of 4,7-di(9H-carbazol-9-yl)-9-oxo-9,10-dihydro-1,10-phenanthroline-5-carbonitrile (6a) and 2-oxo-4,7-di(10H-phenothiazin-10-yl)-1,2-dihydro-1,10-phenanthroline-5-carbonitrile (6b)

Compounds $5 \mathrm{~m}$ or $5 \mathrm{n}(3.5 \mathrm{mmol})$ dissolved in a mixture composed of THF and $10 \%$ sodium hydroxide $(55 \mathrm{~mL})$ were stirred for 4 days at $50^{\circ} \mathrm{C}$. The cooled solution was acidified to neutral $\mathrm{pH}$ with concentrated hydrochloric acid. After solvent evaporation, the crude product was purified by column chromatography on silica gel using methanol/dichloromethane as eluent to afford a white powder, and finally crystallization from the mixture of $\mathrm{CH}_{2} \mathrm{Cl}_{2}$ and hexane to yield solids as follows:

Compound 6a: yellowish $0.6 \mathrm{~g}(1.1 \mathrm{mmol}, 31 \%) ; \mathrm{m} . \mathrm{p}_{\mathrm{dec}} .=195-200{ }^{\circ} \mathrm{C} ;{ }^{1} \mathrm{H}-\mathrm{NMR}\left(\mathrm{CDCl}_{3} ; 400.2\right.$ $\mathrm{MHz}) \delta=6.90\left(\mathrm{~d},{ }^{3} J_{\mathrm{H}, \mathrm{H}}=7.3 \mathrm{~Hz}, 2 \mathrm{H}\right.$, aromatic), $7.14\left(\mathrm{~s}, 1 \mathrm{H}\right.$, aromatic), $7.21\left(\mathrm{~d},{ }^{3} J_{\mathrm{H}, \mathrm{H}}=8.0 \mathrm{~Hz}, 2 \mathrm{H}\right.$, aromatic), 7.29-7.45 (m, 8H, aromatic), $7.65\left(\mathrm{~s}, 1 \mathrm{H}\right.$, aromatic), $7.83\left(\mathrm{~d},{ }^{3} \mathrm{~J}_{\mathrm{H}, \mathrm{H}}=4.3 \mathrm{~Hz}, 1 \mathrm{H}\right.$, aromatic), 8.16-8.17 (m, $4 \mathrm{H}$, aromatic), $9.27\left(\mathrm{~d},{ }^{3} \mathrm{~J}_{\mathrm{H}, \mathrm{H}}=4.3 \mathrm{~Hz}, 1 \mathrm{H}\right.$, aromatic), $11.24(\mathrm{bs}, 1 \mathrm{H}, \mathrm{NH}) ;{ }^{13} \mathrm{C}\left\{{ }^{1} \mathrm{H}\right\}-\mathrm{NMR}$ 
$\left(\mathrm{CDCl}_{3} ; 100.5 \mathrm{MHz}\right) \delta=100.8,109.1,110.0,115.5,115.7,121.0,121.2,121.5,121.7,124.2,124.4,124.5$, 126.3, 126.7, 126.8, 126.9, 133.5, 139.0, 140.1, 140.5, 142.2, 143.6, 146.5, 151.8, 161.7; MS-IT-TOF [M + $\mathrm{H}]^{+}=552.1830(100 \%),[\mathrm{M}-\mathrm{H}]^{-}=550.1668(100 \%)$, HRMS (IT TOF): $m / z$ Calcd for $\mathrm{C}_{37} \mathrm{H}_{22} \mathrm{~N}_{5} \mathrm{O}[\mathrm{M}+$ $\mathrm{H}^{+}=552.1824$, Found 552.1830; UV-Vis (methanol; $\lambda$ [nm] (loge)): 374 (3.46), 355 (3.57), 329 (3.82), 316 (3.76), 292 (4.27), 287 (4.25), 282 (4.27), 247 (4.31), 231 (4.62), 210 (4.33); IR (ATR): $\tilde{v}=3051,2360,2218$, $1675,1446,1226,747,723 \mathrm{~cm}^{-1}$; CCDC 1,917,090 (Supporting Information Figure S28).

Compound 6b: yellowish $1.0 \mathrm{~g}(1.6 \mathrm{mmol}, 46 \%) ; \mathrm{mp}_{\text {dec. }}=220-230{ }^{\circ} \mathrm{C} ;{ }^{1} \mathrm{H}-\mathrm{NMR}\left(\mathrm{CDCl}_{3} ; 400.2\right.$ $\mathrm{MHz}) \delta=5.63\left(\mathrm{~d},{ }^{3} \mathrm{~J}_{\mathrm{H}, \mathrm{H}}=8.2 \mathrm{~Hz}, 2 \mathrm{H}\right.$, aromatic), 6.63-6.58 (m, $2 \mathrm{H}$, aromatic), $6.66\left(\mathrm{dd},{ }^{3} \mathrm{~J}_{\mathrm{H}, \mathrm{H}}=7.8\right.$ $\mathrm{Hz},{ }^{4} J_{\mathrm{H}, \mathrm{H}}=0.9 \mathrm{~Hz}, 2 \mathrm{H}$, aromatic), $6.77\left(\mathrm{t},{ }^{3} J_{\mathrm{H}, \mathrm{H}}=7.5 \mathrm{~Hz}, 2 \mathrm{H}\right.$, aromatic), 6.90-7.00 (m, $6 \mathrm{H}$, aromatic), 7.12-7.18 (m, 2H, aromatic), $7.25\left(\mathrm{~s}, 1 \mathrm{H}\right.$, aromatic), $7.84\left(\mathrm{~d},{ }^{3} J_{\mathrm{H}, \mathrm{H}}=4.5 \mathrm{~Hz}, 1 \mathrm{H}\right.$, aromatic), $8.45(\mathrm{~s}, 1 \mathrm{H}$, aromatic), $9.27\left(\mathrm{~d},{ }^{3} \mathrm{~J}_{\mathrm{H}, \mathrm{H}}=4.6 \mathrm{~Hz}, 1 \mathrm{H}\right.$, aromatic), 11.11 (bs. $\left.1 \mathrm{H}, \mathrm{NH}\right) ;{ }^{13} \mathrm{C}\left\{{ }^{1} \mathrm{H}\right\}-\mathrm{NMR}\left(\mathrm{CDCl}_{3} ; 100.5\right.$ MHz) $\delta=101.0,115.1,116.2,116.7,117.5,119.8,122.6,123.5,124.5,127.0,127.2,127.5,127.9,128.5$, 129.7, 129.8 134.1, 139.6, 141.0, 141.9, 142.1, 146.9, 149.2, 153.0, 161.7; MS-IT-TOF [M + H] $]^{+}=616.1271$ $(100 \%),[\mathrm{M}-\mathrm{H}]^{-}=614.1110(100 \%)$, HRMS (IT TOF): $\mathrm{m} / \mathrm{z}$ Calcd for $\mathrm{C}_{37} \mathrm{H}_{20} \mathrm{~N}_{5} \mathrm{OS}[\mathrm{M}-\mathrm{H}]^{-}=614.1109$, Found 614.1110; UV-Vis (methanol; $\lambda$ [nm] (loge)): 339 (3.94), 295 (4.52), 284 (4.38), 273 (4.18), 251 (4.79), 205 (4.59); IR (ATR): $\tilde{v}=3059,2974,2360,2216,1673,1461,1312,743,648 \mathrm{~cm}^{-1}$; CCDC 1,919,692 (Supporting Information Figure S29).

\subsection{Electrochemical Setup}

Cyclic voltammetry was measured using a potentiostat PGSTAT12 (Metrohm Autolab B.V., Utrecht, The Netherlands) and a three-electrode electrochemical cell with an $\mathrm{Ag}|\mathrm{AgCl}| 1 \mathrm{M} \mathrm{LiCl}$ reference electrode separated from the test solution by a salt bridge. A glassy carbon electrode (with diameter $0.7 \mathrm{~mm}$ ) and a platinum wire were the working and counter electrodes, respectively. Oxygen was removed from the solution by passing a stream of argon. Stock solution of analyte was prepared in degassed anhydrous $99.8 \%$ acetonitrile (ACN, anhydrous, Sigma-Aldrich, content of water $<0.001 \%$ ) supplied under argon. Tetrabutylammonium hexafluorophosphate (Sigma Aldrich) was used as a supporting electrolyte and dried before use.

\subsection{Molecular Orbital Calculations}

Theoretical calculations of frontier molecular orbital energies were performed using the density functional theory (DFT) calculations employing the B3LYP functional and 6-31G* basis set for all atoms with Spartan'14, v.1.1.8 software (Wavefunction, Inc., Irvine, CA USA). The spatial distribution of HOMO and LUMO orbitals was calculated for the geometry optimized molecules in vacuum.

\section{Conclusions}

This research has focused on the synthesis of 27 4,7-disubstituted-1,10-phenanthrolines, including their 4,7-dichloro-, 4,7-di(9H-carbazol-9-yl)-, 4,7-di(10H-phenothiazin-10-yl)- and 4,7-di(pyrrolidin-1-yl) derivatives, giving 23 novel compounds. The presented protocols allowed us to synthesize the targeted compounds more efficiently, with yields up to $96 \%$. The structures of the obtained molecules were proved by a combination of varies techniques, such as NMR, GC-MS, MS, HRMS, UV-Vis and X-ray crystallography. A variety of substituents (methyl, halogen (F, Cl and $\mathrm{Br}$ ), $\mathrm{CN}, 9 \mathrm{H}$-carbazole, pyrrolidine, $10 \mathrm{H}$-phenothiazine, $\mathrm{COOEt}$ and $\mathrm{COOH}$ groups) were chosen in order to represent different electronic features. For the first time ${ }^{15} \mathrm{~N} \mathrm{CP} / \mathrm{MAS}-\mathrm{NMR}$ spectra of selected 4,7-disubstituted-1,10-phenanthroline derivatives were elucidated to differentiate the nitrogen nucleus and to give an insight into their characteristics. The electrochemical studies showed the influence of substituents on the redox properties of synthesized compounds. Compounds with methyl as $\mathbf{R}$ substituent were the most difficult ones to reduce. On the contrary, compounds substituted with $9 H$-carbazole as $\mathbf{R}^{2}$ had the highest oxidation potentials and were the most stable ones against oxidative processes. Compounds substituted with phenothiazine and pyrrolidine as $\mathbf{R}^{\mathbf{2}}$ were the most easily oxidized due to the oxidation of the substituent $\mathbf{R}^{2}$. Phenothiazine derivatives were also stronger electron acceptors and were more facile 
to reduction than other compounds. Regarding the largest potential gap, methylated compounds $\mathbf{4 g}$, $4 \mathbf{k}$ and compound $\mathbf{5 f}$ containing $9 \mathrm{H}$-carbazole are the most stable structures against oxidative and reductive processes.

Supplementary Materials: The following are available online at http:/www.mdpi.com/1420-3049/24/22/4102/ s1. CCDC 1479401 for 5d, CCDC 1917090 for 6a and CCDC 1919692 for $6 \mathbf{b}$ contains the supplementary crystallographic data for this paper. These data can be obtained free of charge via http://www.ccdc.cam.ac. uk/conts/retrieving.html (or from the CCDC, 12 Union Road, Cambridge CB2 1EZ, UK; Fax: +44 1223 336033; E-mail: deposit@ccdc.cam.ac.uk)". This text may be included in the experimental section or as a suitably referenced endnote.

Author Contributions: J.E.N., J.W., M.S. (Marek Stankevič) and D.S. performed the experiments; J.G.M. X-ray measurements and diffraction data analysis, wrote chapter; J.W. and R.S. electrochemistry data analysis, wrote chapter; R.S. and J.G.M. theoretical calculations (DFT); M.S. (Marcin Szala) IR and UV-Vis measurements; L.P. NMR in solid state measurements; J.E.N. designed and analyzed the data, wrote manuscript.

Funding: Romana Sokolova greatly acknowledges the Czech Academy of Sciences (RVO: 61388955).

Acknowledgments: We thank the Erasmus Plus and "CIS- Chemia i Staże" for the 9 months award to stay at J. Heyrovský Institute of Physical Chemistry of the Czech Academy of Sciences, Dolejskova 3, 18223, Prague, Czech Republic to J. Wantulok.

Conflicts of Interest: The authors declare no conflict of interest.

\section{References}

1. Gladiali, S.; Chelucci, G.; Soccolini, F.; Delogu, G.; Chessa, G. Optically active phenanthrolines in asymmetric catalysis. II. Enantioselective transfer hydrogenation of acetophenone by rhodium/alkyl phenanthroline catalysts. J. Organomet. Chem. 1989, 370, 285-294. [CrossRef]

2. Sammes, P.G.; Yahioglu, G. 1,10-Phenanthroline: A versatile ligand. Chem. Soc. Rev. 1994, 23, 327-334. [CrossRef]

3. Monnier, F.; Taillefer, M. Catalytic C-C, C-N, and C-O Ullmann-type coupling reactions. Angezw. Chem. Int. Ed. 2009, 48, 6954-6971. [CrossRef] [PubMed]

4. Feist, B.; Pilch, M.; Nycz, J. Graphene oxide chemically modified with 5-amino-1,10-phenanthroline as sorbent for separation and preconcentration of trace amount of lead(II). Microchim. Acta 2019, 186, 91. [CrossRef] [PubMed]

5. Chambron, J.-C.; Collin, J.-P.; Heitz, V.; Jouvenot, D.; Kern, J.-M.; Mobian, P.; Pomeranc, D.; Sauvage, J.-P. Rotaxanes and catenanes built around octahedral transition metals. Eur. J. Org. Chem. 2004, 2004, 1627-1638. [CrossRef]

6. Hamann, C.; Kern, J.-M.; Sauvage, J.-P. Zinc(II)-templated synthesis of a [2]-catenane consisting of a 2,2', $6^{\prime}, 2^{\prime}$-terpyridine-incorporating cycle and a 1,10-phenanthroline-containing ring. Inorg. Chem. 2003, 42, 1877-1883. [CrossRef]

7. Bencini, A.; Lippolis, V. 1,10-Phenanthroline: A versatile building block for the construction of ligands for various purposes. Coord. Chem. Rev. 2010, 254, 2096-2180. [CrossRef]

8. Accorsi, G.; Listorti, A.; Yoosaf, K.; Armaroli, N. 1,10-Phenanthrolines: Versatile building blocks for luminescent molecules, materials and metal complexes. Chem. Soc. Rev. 2009, 38, 1690-1700. [CrossRef]

9. Hara, K.; Sugihara, H.; Tachibana, Y.; Islam, A.; Yanagida, M.; Sayama, K.; Arakawa, H.; Fujihashi, G.; Horiguchi, T.; Kinoshita, T. Dye-sensitized nanocrystalline $\mathrm{TiO}_{2}$ solar cells based on ruthenium(II) phenanthroline complex photosensitizers. Langmuir 2001, 17, 5992-5999. [CrossRef]

10. Bai, Y.; Yu, Q.J.; Cai, N.; Wang, Y.H.; Zhang, M.; Wang, P. High-efficiency organic dye-sensitized mesoscopic solar cells with a copper redox shuttle. Chem. Commun. 2011, 47, 4376-4378. [CrossRef]

11. Hu, Z.-Q.; Hu, H.-Y.; Chen, C.-F. Phenanthroline Dicarboxamide-Based Helical Foldamers: Stable Helical Structures in Methanol. J. Org. Chem. 2006, 71, 1131-1138. [CrossRef] [PubMed]

12. Williams, N.J.; Dean, N.E.; Van Derveer, D.G.; Luckay, R.C.; Hancock, R.D. Strong metal ion size based selectivity of the highly preorganized ligand PDA (1,10-phenanthroline-2.9-dicarboxylic acid) with trivalent metal ions. A crystallographic, fluorometric, and thermodynamic study. Inorg. Chem. 2009, 48, 7853-7863. [CrossRef] [PubMed] 
13. Shao, J.; Qiao, Y.-H.; Lin, H.; Lin, H.-K. A turn-on fluorescent anion receptor based on N,N'-di- $\beta$-naphthyl-1,10-phenanthroline-2,9-diamide. J. Lumin. 2008, 128, 1985-1988. [CrossRef]

14. Huang, C.-H.; Parish, A.; Samain, F.; Garo, F.; Häner, R.; Morrow, J.R. Binding of europium(iii) to a non-nucleosidic phenanthroline linker in DNA. Bioconjugate Chem. 2010, 21, 476-482. [CrossRef]

15. Gude, L.; Fernández, M.J.; Grant, K.B.; Lorente, A. Syntheses and copper(II)-dependent DNA photocleavage by acridine and anthracene 1,10-phenanthroline conjugate systems. Org. Biomol. Chem. 2005, 3, 1856-1862. [CrossRef]

16. Ou, T.-M.; Lu, Y.-J.; Tan, J.-H.; Huang, Z.-S.; Wong, K.-Y.; Gu, L.-Q. G-quadruplexes: Targets in anticancer drug design. ChemMedChem 2008, 3, 690-713. [CrossRef]

17. Ye, R.-R.; Tan, C.-P.; Lin, Y.-N.; Ji, L.-N.; Mao, Z.-W. A phosphorescent rhenium(I) histone deacetylase inhibitor: Mitochondrial targeting and paraptosis induction. Chem. Commun. 2015, 51, 8353-8356. [CrossRef]

18. Wang, L.; Wen, Y.; Liu, J.; Zhou, J.; Li, C.; Wei, C. Promoting the formation and stabilization of human telomeric G-quadruplex DNA, inhibition of telomerase and cytotoxicity by phenanthroline derivatives. Org. Biomol. Chem. 2011, 9, 2648-2653. [CrossRef]

19. Reed, J.E.; Neidle, S.; Vilar, R. Stabilisation of human telomeric quadruplex DNA and inhibition of telomerase by a platinum-phenanthroline complex. Chem. Commun. 2007, 4366-4368. [CrossRef]

20. Nielsen, M.C.; Borch, J.; Ulven, T. Design, synthesis and evaluation of 4,7-diamino-1,10-phenanthroline G-quadruplex ligands. Bioorg. Med. Chem. 2009, 17, 8241-8246. [CrossRef]

21. Monchaud, D.; Teulade-Fichou, M.P. A hitchhiker's guide to G-quadruplex ligands. Org. Biomol. Chem. 2008, 6, 627-636. [CrossRef]

22. Larsen, A.F.; Ulven, T. Efficient synthesis of 4,7-diamino substituted 1,10-phenanthroline-2,9-dicarboxamides. Org. Lett. 2011, 13, 3546-3548. [CrossRef] [PubMed]

23. Graf, G.I.; Hastreiter, D.; da Silva, L.E.; Rebelo, R.A.; Montalban, A.G.; McKillop, A. The synthesis of aromatic diazatricycles from phenylenediamine-bis(methylene Meldrum's acid) derivatives. Tetrahedron 2002, 58, 9095-9100. [CrossRef]

24. Engel-Andreasen, J.; Shimpukade, B.; Ulve, T. Selective copper catalysed aromatic N-arylation in water. Green Chem. 2013, 15, 336-340. [CrossRef]

25. Zucchi, G.; Murugesan, V.; Tondelier, D.; Aldakov, D.; Jeon, T.; Yang, F.; Thuéry, P.; Ephritikhine, M.; Geffroy, B. Solution, solid state, and film properties of a structurally characterized highly luminescent molecular europium plastic material excitable with visible light. Inorg. Chem. 2011, 50, 4851-4856. [CrossRef]

26. Shaffer, K.J.; Parr, D.C.; Wenzel, M.; Rowlands, G.J.; Plieger, P.G. The proton sponge effect: Substitution of quino[7,8-h]quinoline and the first structurally characterised derivatives. Eur. J. Org. Chem. 2012, 2012, 6967-6975. [CrossRef]

27. Ten Brink, G.-J.; Arends, I.W.C.E.; Hoogenraad, M.; Verspui, G.; Sheldon, R.A. Catalytic Conversions in Water. Part 23: Steric Effects and Increased Substrate Scope in the Palladium-Neocuproine Catalyzed Aerobic Oxidation of Alcohols in Aqueous Solvents. Adv. Synth. Catal. 2003, 345, 1341-1352. [CrossRef]

28. Wu, K.; Zhang, T.; Zhan, L.; Zhong, C.; Gong, S.; Lu, Z.-H.; Yang, C. Tailoring Optoelectronic Properties of Phenanthroline-Based Thermally Activated Delayed Fluorescence Emitters through Isomer Engineering. Adv. Optical Mater. 2016, 4, 1558-1566. [CrossRef]

29. Moutiers, G.; Pinson, J.; Terrier, F.; Goumont, R. Electrochemical oxidation of $\sigma$-complex-type intermediates in aromatic nucleophilic substitutions. Chem. Eur. J. 2001, 7, 1712-1719. [CrossRef]

30. Gallardo, I.; Guirado, G.; Marquet, J. Nucleophilic Aromatic Substitution of Hydrogen: A Novel Electrochemical Approach to the Cyanation of Nitroarenes. Chem. Eur. J. 2001, 7, 1759-1765. [CrossRef]

31. CrysAlisPro 1.171.38.43 (Rigaku Oxford Diffraction, 2015). Available online: https://www.rigaku.com/en/ products/smc/crysalis (accessed on 20 October 2019).

32. Dolomanov, O.V.; Bourhis, L.J.; Gildea, R.J.; Howard, J.A.K.; Puschmann, H. OLEX2: A complete structure solution, refinement and analysis program. J. Appl. Cryst. 2009, 42, 339-341. [CrossRef]

33. Sheldrick, G.M. A short history of SHELX. Acta Cryst. 2008, A64, 112-122. [CrossRef] [PubMed]

34. Groen, J.H.; de Jong, B.J.; Ernsting, J.-M.; van Leeuwen, P.W.N.M.; Vrieze, K.; Smeets, W.J.J.; Spek, A.L. Synthesis, characterization and reactivity of ionic palladium(II) complexes containing bidentate nitrogen ligands in a unidentate coordination mode ${ }^{1}{ }^{2}$. J. Organomet. Chem. 1999, 573, 3-13. [CrossRef] 
35. Pawlak, T.; Pazderski, L.; Sitkowski, J.; Kozerski, L.; Szłyka, E. ${ }^{1} \mathrm{H},{ }^{13} \mathrm{C},{ }^{195} \mathrm{Pt}$ and ${ }^{15} \mathrm{~N}$ NMR structural correlations in $\mathrm{Pd}(\mathrm{II})$ and $\mathrm{Pt}(\mathrm{II})$ chloride complexes with various alkyl and aryl derivatives of 2,2' -bipyridine and 1,10-phenanthroline. Magn. Reson. Chem. 2011, 49, 59-64. [CrossRef] [PubMed]

36. Peters, D.G. Halogenated Organic Compounds in Organic Electrochemistry; Lund, H., Hammerich, O., Eds.; Marcel Dekker, Inc.: New York, NY, USA, 2001; pp. 341-377.

37. Sokolova, R.; Gal, M.; Valasek, M. New proton donors in electrochemical mechanistic studies in non-aqueous solution dimethylsulfoxide: Chlorinated hydroxybenzonitriles. J. Electroanal. Chem. 2012, 685, 33-36. [CrossRef]

38. Gayathri, P.; Kumar, A.S. Electrochemical behavior of the 1,10-phenanthroline ligand on a multiwalled carbon nanotube surface and its relevant electrochemistry for selective recognition of copper ion and hydrogen peroxide sensing. Langmuir 2014, 30, 10513-10521. [CrossRef]

39. Hayen, H.; Karst, U. Analysis of phenothiazine and its derivatives using LC/electrochemistry/MS and LC/electrochemistry/fluorescence. Anal. Chem. 2003, 75, 4833-4840. [CrossRef]

40. Mielech-Łukasiewicz, K.; Puzanowska-Tarasiewicz, H.; Panuszko, A. Electrochemical oxidation of phenothiazine derivatives at glassy carbon electrodes and their differential pulse and square-wave voltammetric determination in pharmaceuticals. Anal. Lett. 2008, 41, 789-805. [CrossRef]

41. Junzo, H.; Hiroyuki, M.; Kenji, H.; Kiyoshi, Z. A ratiometric fluorescent chemosensor, 1,10-phenanthroline-4,7-dione, for anions in aqueous-organic media. Tetrahedron Lett. 2007, 48, 4861-4864.

42. Świtlicka, A.; Klemens, T.; Machura, B.; Schab-Balcerzak, E.; Laba, K.; Lapkowski, M.; Grucela, M.; Nycz, J.; Szala, M.; Kania, M. Rhenium(i) complexes with phenanthrolines bearing electron-withdrawing $\mathrm{Cl}$ and electron-donating $\mathrm{CH}_{3}$ substituents - synthesis, photophysical, thermal, and electrochemical properties with electroluminescence ability. RSC Adv. 2016, 6, 112908-112918. [CrossRef]

43. Belian, M.F.; Batista, H.J.; Bezerra, A.G.S.; Silva, W.E.; de Sá, G.F.; Alves Jr., S. Eu(III) complex luminescence behavior upon chlorine substitution in the 1,10-phenanthroline ligand: A theoretical and experimental study. Chem. Phys. 2011, 381, 29-34. [CrossRef]

44. Maron, A.; Nycz, J.E.; Machura, B.; Małecki, J.G. Luminescence properties of palladium(II) phenanthroline derivative coordination compounds. ChemistrySelect 2016, 4, 798-804. [CrossRef]

45. Edwards, A.C.; Wagner, C.; Geist, A.; Burton, N.A.; Sharrad, C.A.; Adams, R.W.; Pritchard, R.G.; Panak, P.J.; Whitehead, R.C.; Harwood, L.M. Exploring electronic effects on the partitioning of actinides(III) from lanthanides(III) using functionalised bis-triazinyl phenanthroline ligands. Dalton T. 2016, 45, 18102-18112. [CrossRef] [PubMed]

46. Edwards, Y.A.C.; Geist, A.; Müllich, U.; Sharrad, C.A.; Pritchard, R.G.; Whitehead, R.C.; Harwood, L.M. Transition metal-free, visible-light mediated synthesis of 1,10-phenanthroline derived ligand systems. Chem. Commun. 2017, 53, 8160-8163. [CrossRef]

Sample Availability: Samples of the compounds $4 a, 4 b, 4 d, 4 g, 4 i, 4 k, 5 f, 5 g, 5 h, 5 i, 5 j, 5 k$ and $6 a$ are available from the authors.

(C) 2019 by the authors. Licensee MDPI, Basel, Switzerland. This article is an open access article distributed under the terms and conditions of the Creative Commons Attribution (CC BY) license (http://creativecommons.org/licenses/by/4.0/). 